\title{
The Role of 2‘,3'-Cyclic Nucleotide 3'-Phosphodiesterase (CNP) in the Peripheral Nervous System
}

\author{
Dissertation \\ for the award of the degree \\ "Doctor rerum naturalium" (Dr.rer.nat) \\ of the Georg-August University Göttingen \\ within the doctoral program Biology \\ of the Georg-August University School of Science (GAUSS) \\ submitted by \\ Theresa Kungl \\ from Weißenfels, Germany
}

Göttingen, 2014 


\section{Examination board}

Prof. Klaus-Armin Nave Ph.D. (Reviewer)

Department of Neurogenetics

Max Planck Institute of Experimental Medicine, Göttingen

Prof. Dr. Ernst A. Wimmer (Reviewer)

Department of Developmental Biology

Johann-Friedrich-Blumenbach-Institute of Zoology and Anthropology (GZMB)

Georg-August University Göttingen

Prof. Dr. Michael W. Sereda

Research group of Molecular and Translational Neurology

Department of Neurogenetics

Max Planck Institute of Experimental Medicine, Göttingen

Prof. Dr. Moritz J. Rossner

Research group of Molecular Neurobiology

Department of Psychiatry and Psychotherapy

Ludwig-Maximilians University of Munich

Prof. Dr. Nils Brose

Department of Molecular Neurobiology

Max Planck Institute of Experimental Medicine, Göttingen

Dr. Manuela Schmidt

Research group of Somatosensory Signaling

Max Planck Institute of Experimental Medicine, Göttingen

Date of oral examination: 20.10 .2014 


\section{Declaration}

I declare that the $\mathrm{PhD}$ thesis entitled "The Role of $2^{\prime}, 3^{\prime}$-Cyclic Nucleotide 3'Phosphodiesterase (CNP) in the Peripheral Nervous System" was written independently and with no other sources and aids than quoted.

Göttingen, 
The more I learn, the more I realize

how much I don't know.

Albert Einstein

To my family,

especially my father and mother. 


\section{Acknowledgements}

First of all I would like to thank my supervisors Prof. Klaus-Armin Nave and Prof. Dr. Michael W. Sereda for allowing me to work independently on this interesting project, to develop ideas and for the possibility to use so many different techniques during the course of the project. Thank you for your help in developing ideas and all the valuable discussions.

I further want to thank my additional thesis committee members Prof. Dr. Ernst A. Wimmer and Prof. Dr. Moritz J. Rossner who further supported me during my thesis with fruitful discussions. Moreover, I would like to thank Prof. Dr. Nils Brose and Dr. Manuela Schmidt for your willingness to participate in the defense.

\section{Many Thanks also to ...}

...the whole PNS group, especially Ruth, Susanne, Bastian and Robert for all the good scientific discussions and that you helped me with project development. I also would like to thank Tobias and Sabrina because you taught me so many things when I started working here. Special thanks to Susanne, Madlen and Nadja for taking care of our glucose supply.

...Michaela and Gabriele for all your help with the organizational things, it would have been a mess without you!

...Torben, Annette, Ulli, Ramona, Christian, Tarik, Elena and Jenni for your support with all the little and big things in daily lab-life!

...Wiebke and Torben for introducing and helping me with all the electron microscopy.

...Matthieu and Liam for all your help and advices.

Riesengroßer Dank auch an meine Eltern, für Eure mentale Unterstützung und Eure Geduld während der gesamten Doktorarbeitszeit! Ebenso großer Dank geht auch an meinen Bruder für die vielen angeregten Diskussionen und Deine selbstverständliche Hilfe. Ich danke Euch und auch meiner ganzen restlichen Familie. ...Geschafft!

...my girls Lotti, Hannah, Ulrike, Sarah, Elina and Michaela. Thanks for all your help and support, all the fruitful discussions and your constantly open ears. Thanks for all the great things that we did besides working. Thanks also for all the tasty meals you served every day. You made my time in the lab really splendid.

... all of you who helped me with correcting and proofreading this work!

Many thanks to the whole Neurogenetics department it's fun working with you! 


\section{Content}

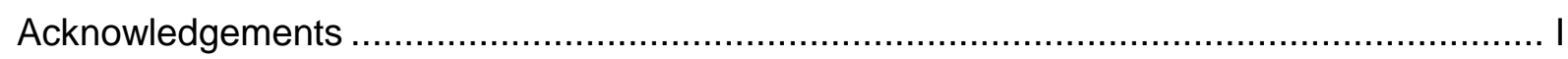

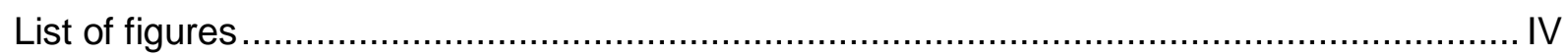

Abbreviations

Summary

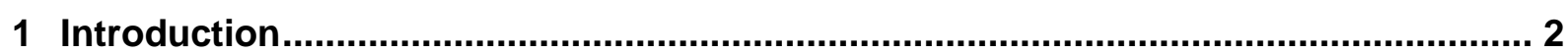

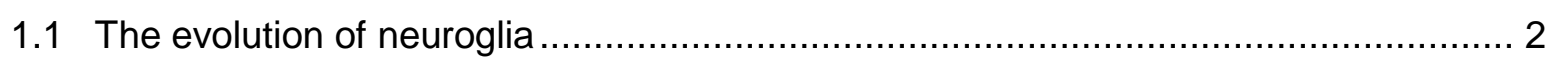

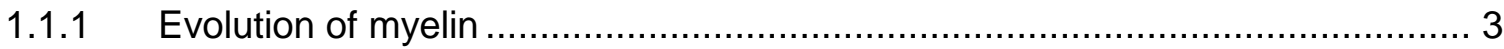

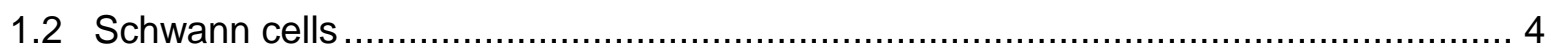

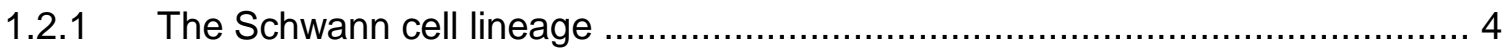

1.2.2 Regulation of Schwann cell development ............................................... 5

1.2.3 Regulation of myelin sheath thickness ....................................................... 5

1.2.4 Noncompact myelin and the axon-glia interaction ...................................... 7

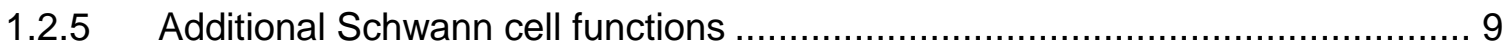

1.2.6 Different myelinating Schwann cell types ...............................................10

1.3 2',3'-Cyclic Nucleotide 3'-Phosphodiesterase (CNP) ........................................11

1.3.1 Molecular characteristics of CNP..........................................................

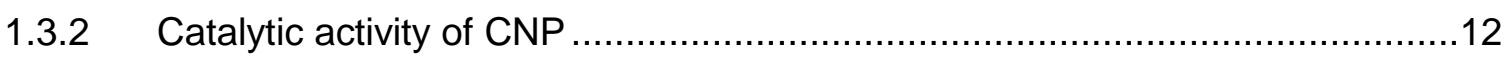

1.3.3 CNP functions independent of the catalytic activity ...................................12

1.3.4 Overexpression and deficiency of CNP ...............................................13

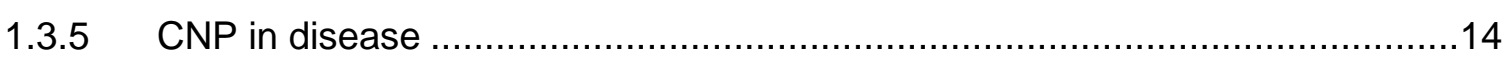

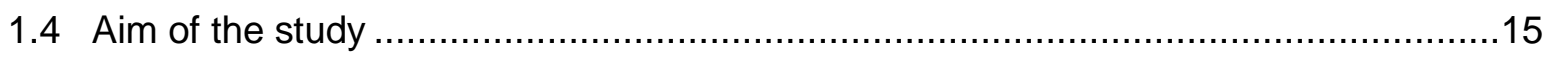

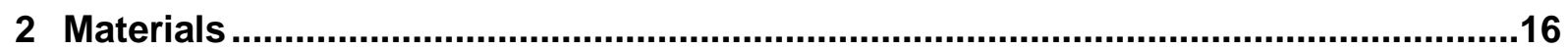

2.1 Chemicals and consumables ...................................................................... 16

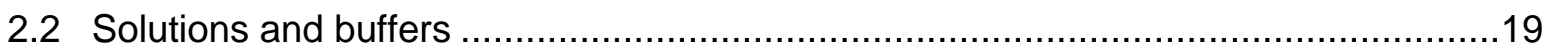

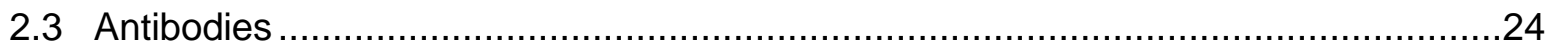

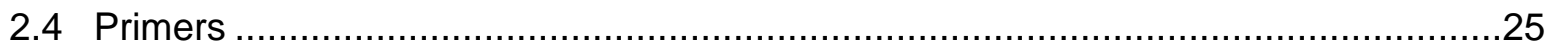

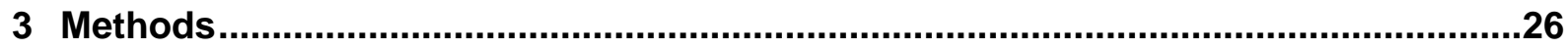

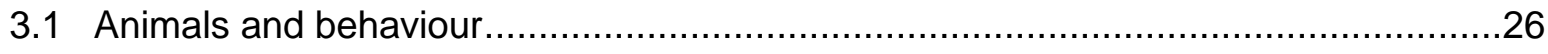

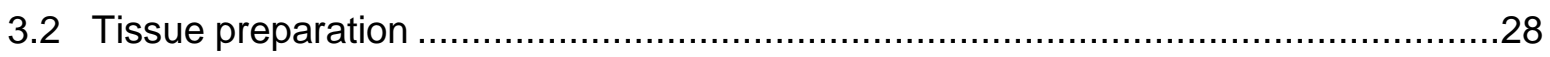

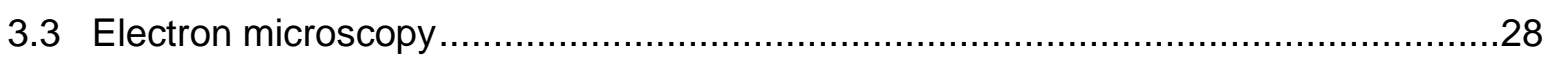

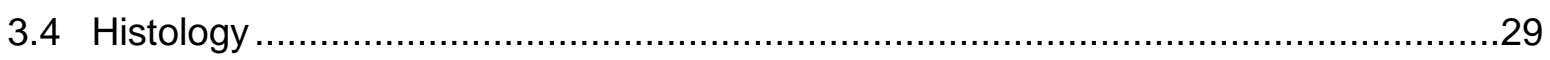



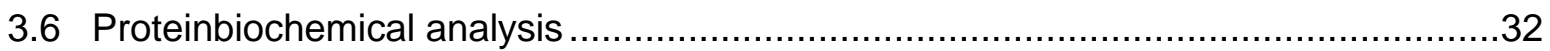

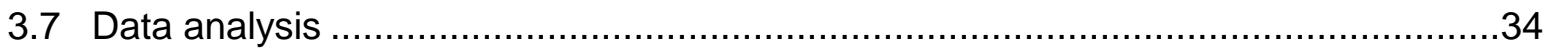

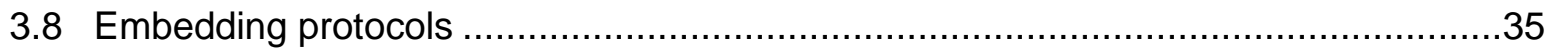




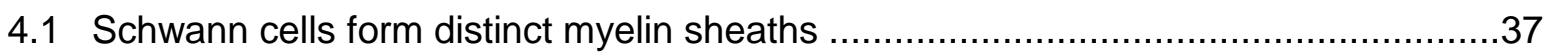

4.1.1 Distinct myelin composition in dorsal and ventral roots..............................38

4.1.2 Different abundance of myelin proteins in dorsal and ventral roots ..................40

4.2 CNP is elevated in myelinating Schwann cells of dorsal roots ................................42

4.2.1 Histological confirmation of increased CNP levels in dorsal roots....................42

4.2.2 CNP transcript levels are enhanced in dorsal root Schwann cells ..................43

4.2.3 Nonmyelinating Schwann cells do not express substantial CNP levels............43

4.3 Ablation of CNP influences the noncompact myelin compartment...........................45

4.3.1 Paranodal loops and Schmidt-Lanterman incisures remain unaltered .............45

4.3.2 Inner tongues are smaller in the PNS of adult CNP deficient mice ................47

4.3.3 Cytoplasmic channels are less frequent in adult CNP mutants.......................49

4.3.4 The inner mesaxon is fully compacted in CNP mutants ................................50

4.4 CNP deficiency causes hypermyelination of small fibers ......................................52

4.4.1 Adult onset hypermyelination of peripheral nerves in CNP mutant mice ..........52

4.4.2 Unaltered myelin periodicity in CNP deficient animals .................................53

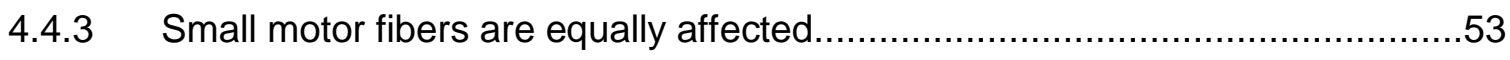

4.4.4 No evidence for focal hypermyelination or myelination threshold shift .............56

4.4.5 Molecular alterations caused by the CNP deficiency ....................................57

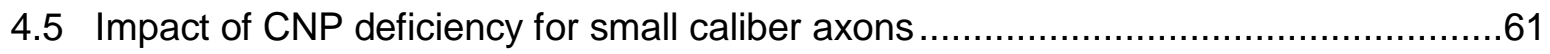

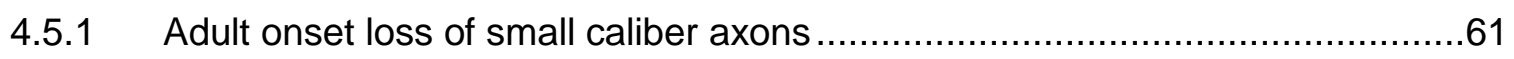

4.5.2 Remak bundles remain unaffected by CNP deficiency ..................................64

4.5.3 No indication of inflammation within peripheral nerves of CNP mutants ..........65

4.5.4 CNP deficient animals display altered sensory nerve characteristics..............66

4.5.5 CNP mutants display impaired sensation ................................................66

5 Discussion .........................................................................................................70

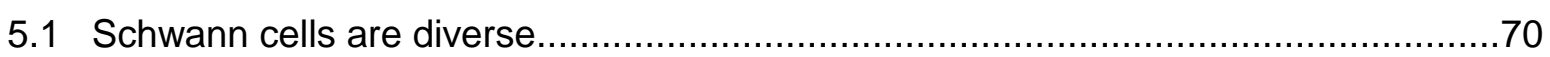

5.2 CNP is enriched in SC surrounding small caliber axons ......................................72

5.3 CNP has an impact on the noncompact and compact myelin compartment .............73

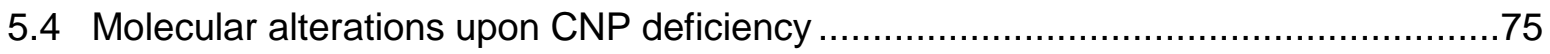

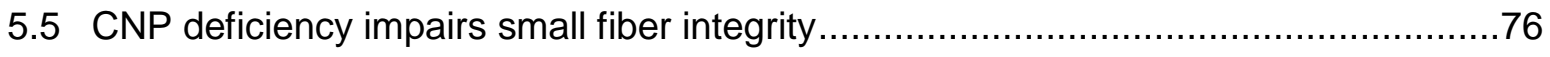

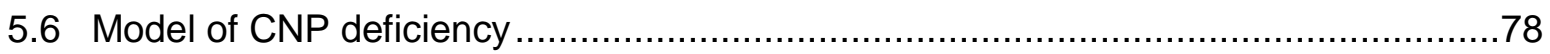

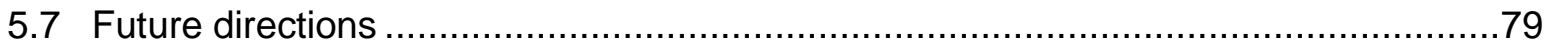

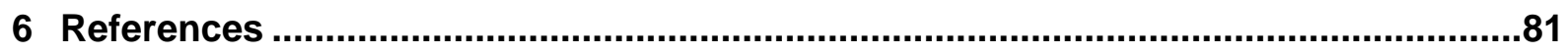




\section{List of figures}

Fig I: $\quad$ Myelin structure in vertebrates and invertebrates................................................ 3

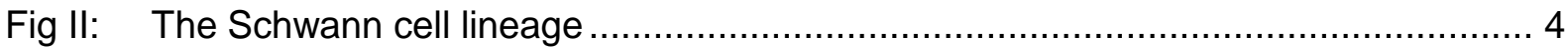

Fig III: Scheme of a myelinated peripheral axon with unrolled sheath .............................. 7

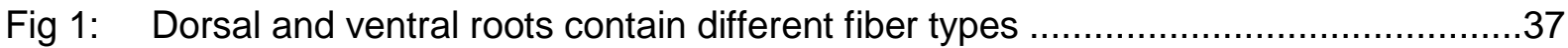

Fig 2: Purified myelin of dorsal and ventral roots displays different protein compositions..39

Fig 3: Distinct myelin protein abundances in dorsal and ventral roots ............................41

Fig 4: Staining intensity for CNP is higher in dorsal roots..........................................42

Fig 5: CNP transcript levels are elevated in dorsal roots................................................43

Fig 6: Nonmyelinating Schwann cells show no considerable CNP expression ...................44

Fig 7: Unaltered paranodal loops and Schmidt-Lanterman incisures in CNP-/- animals....46

Fig 8: Smaller inner tongues in adult CNP mutants................................................. 48

Fig 9: Cytoplasmic channels are less frequent in adult CNP mutants ............................49

Fig 10: The inner mesaxon is more compacted in CNP deficient animals .........................51

Fig 11: Hypermyelination of small caliber axons in CNP deficient mice ...........................55

Fig 12: No focal hypermyelination and unaltered myelination threshold ............................56

Fig 13: Myelin specific mRNA profiles are unaltered when CNP is ablated ........................57

Fig 14: Activation of MAPK and AKT signaling in CNP deficient nerves............................59

Fig 15: Increase of myelin proteins and lipid related genes in adult CNP-/- mice ................60

Fig 16: CNP deficiency causes adult onset sensory axonal loss ....................................62

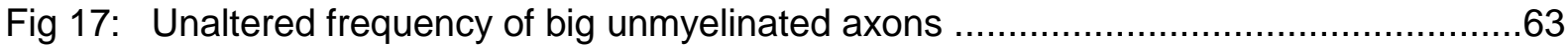

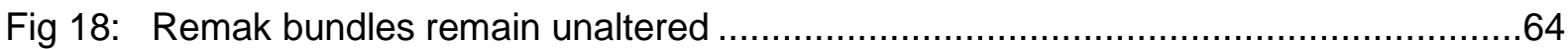

Fig 19: No indication of inflammation within the PNS of CNP deficient animals ..................65

Fig 20: Altered electrophysiological characteristics of CNP deficient peripheral nerves ......67

Fig 21: Impaired sensation and higher order motor coordination ..................................68

Fig 22: Model of SC diversity and the differential impact of CNP on distinct PNS fibers .....78 


\section{Abbreviations}

\begin{tabular}{|c|c|}
\hline ALS & Amyotrophic lateral sclerosis \\
\hline APS & Ammonium persulfate \\
\hline BDNF & Brain-derived neurotrophic factor \\
\hline Bp & Base pairs \\
\hline BSA & Bovine serum albumin \\
\hline${ }^{\circ} \mathrm{C}$ & Degrees Celsius \\
\hline Caspr & Contactin-associated protein \\
\hline CNP & 2',3'-Cyclic nucleotide 3'-phosphodiesterase \\
\hline CNS & Central nervous system \\
\hline Cx29 & Connexin 29 \\
\hline DAB & Diaminobenzidine \\
\hline DAPI & 4'-6-Diamidino-2-phenylindole \\
\hline $\mathrm{ddH}_{2} \mathrm{O}$ & Clean water \\
\hline DR & Dorsal root \\
\hline ECM & Extracellular matrix \\
\hline EDTA & Ethylenediaminetetraacetic acid \\
\hline EM & Electron microscopy \\
\hline ERK & Extracellular signaling regulated kinase \\
\hline GAPDH & Glycerin aldehyde 3-phosphate dehydrogenase \\
\hline GS & Goat serum \\
\hline HS & Horse serum \\
\hline IGF1 & Insulin-like growth factor 1 \\
\hline $\mathrm{IHC}$ & Immunohistochemistry \\
\hline IL & Internodal length \\
\hline $\mathrm{kDa}$ & Kilodalton \\
\hline KO & Constitutive mutant \\
\hline Krox20 & Early growth response protein 2 (Egr2) \\
\hline M & Molar \\
\hline MAG & Myelin associated glycoprotein \\
\hline MAPK & Mitogene activated protein kinase \\
\hline MBP & Myelin basic protein \\
\hline MDL & Major dense line \\
\hline MEK1DD & Gain of function Mek1 (MAPK signaling pathway) \\
\hline$\mu \mathrm{M}$ & Micromolar \\
\hline $\mathrm{mM}$ & Millimolar \\
\hline $\mathrm{mA}$ & Milliampere \\
\hline $\min$ & Minute \\
\hline $\mathrm{ml}$ & Milliliter \\
\hline MS & Mass spectrometry \\
\hline$\mu g$ & Microgram \\
\hline$\mu l$ & Microliter \\
\hline$\mu \mathrm{m}$ & Micrometer \\
\hline MyP2 & Peripheral myelin protein 2 \\
\hline$n$ & Number of samples \\
\hline NFkB & Nuclear factor kappa-light-chain-enhancer of activated B ce \\
\hline ng & Nanogram \\
\hline
\end{tabular}




\begin{tabular}{|c|c|}
\hline $\mathrm{nm}$ & Nanometer \\
\hline Nrg & Neuregulin \\
\hline n.s. & Non-significant \\
\hline Oct6 & POU domain, class 3 , transcription factor 1 (POU3F1) \\
\hline OD & Oligodendrocytes \\
\hline $\mathrm{o} / \mathrm{n}$ & Over night \\
\hline $\mathrm{p}$ & P-value \\
\hline$P$ & Postnatal day \\
\hline P0 & Myelin protein zero \\
\hline PAGE & Polyacrylamid gel electrophorese \\
\hline PBS & Phosphate buffered saline \\
\hline PCR & Polymerase chain reaction \\
\hline PFA & Paraformaldehyde \\
\hline $\mathrm{PI3K}$ & Phosphoinositide-3 kinase \\
\hline PLP & Proteolipid protein \\
\hline PMP22 & Peripheral myelin protein 22 \\
\hline PNS & Peripheral nervous system \\
\hline qRT-PCR & Quantitative real time PCR \\
\hline RNA & Ribonucleic acid \\
\hline Rpm & Rounds per minute \\
\hline$R Q$ & Relative quantity \\
\hline RT & Room temperature \\
\hline $\mathrm{s}$ & Seconds \\
\hline SC & Schwann cell(s) \\
\hline SCAP & SREBP cleavage activating protein \\
\hline SCP & Schwann cell precursor \\
\hline SD & Standard deviation \\
\hline SDS & Sodium dodecyl sulfate \\
\hline SEM & Standard error of the mean \\
\hline Sept2 & Septin 2 \\
\hline SLI & Schmidt-Lanterman incisures \\
\hline Sox10 & SRY-related HMG-box transcription factor 10 \\
\hline SREBP & Sterol regulatory element binding protein \\
\hline TBS & Tris buffered saline \\
\hline TEMED & Tetramethylendiamin \\
\hline WT & Wildtype (control) \\
\hline$w / v$ & Weight per volume \\
\hline U & Unit (for enzyme activities) \\
\hline UV & Ultraviolet \\
\hline V & Volt \\
\hline VR & Ventral root \\
\hline $\mathrm{v} / \mathrm{v}$ & Volume per volume \\
\hline
\end{tabular}




\section{Summary}

In the peripheral nervous system (PNS) Schwann cells (SC) engulf, insulate and support motor and sensory axons. Nonmyelinating SC are associated with axons smaller than $1 \mu \mathrm{m}$ in diameter, whereas myelinating SC form a compact myelin sheath around axons larger than $1 \mu \mathrm{m}$ in diameter to enable rapid saltatory impulse propagation. During peripheral nerve regeneration motor and sensory SC express distinct trophic factors and extracellular matrix components to allow appropriate reinnervation. This implies that motor and sensory SC display a differential molecular architecture also in the intact PNS. It was unknown if differences exist with regard to the myelin sheath. Therefore, this study first aimed to identify differences between motor and sensory SC with respect to their myelin sheath composition. For this purpose, comparative proteome analyses of dorsal and ventral root myelin were performed. Numerous differences in the molecular myelin constitution, beyond the differential expression of classical myelin proteins, were observed. In particular, the noncompact myelin protein 2',3'-cyclic nucleotide 3-phosphodiesterase (CNP) displayed substantially higher expression levels in dorsal compared to ventral roots. This suggests a differential impact of CNP for motor and sensory fibers. In the central nervous system (CNS) CNP is involved in axonal support independent of myelination and its deficiency causes a severe neurodegeneration. Hence, this study further aimed to uncover the role of CNP in the PNS and its importance for different fiber types. Immunostainings revealed that CNP is enriched in myelinating SC that ensheath small caliber motor and sensory axons. As in the CNS, CNP ablation caused a reduction in size of the noncompacted myelin compartment. Moreover, CNP deficient mice exhibited an adult onset hypermyelination of small caliber peripheral nerve fibers. In order to understand the underlying mechanism of continued myelination, MAPK/ERK and PI3K/AKT signaling pathways were investigated. Surprisingly, the activities of both pathways increased in the absence of CNP, suggesting that CNP is a negative regulator of both pathways in mature nerves. Together, CNP deficiency caused a remarkable imbalance of the noncompacted and compacted myelin compartments which was most pronounced in SC of small fibers. Noncompacted myelin is thought to serve as a transport network for trophic factors and metabolites. Therefore the reduction of this compartment may hamper axonal support, which may have caused the observed loss of predominantly small caliber axons in adult CNP deficient mice. Taken together, CNP plays a role in axonal support and postdevelopmental negative regulation of myelination and is crucial for the integrity of small to mid-sized caliber axons in the PNS. Moreover, myelinating SC display individual myelin sheath compositions according to the size of the underlying axon, which may resemble precise adjustments to axonal demands. Understanding the myelin biology of different fibers may contribute to our understanding of motor and sensory peripheral neuropathies. 


\section{Introduction}

\subsection{The evolution of neuroglia}

An important step in the evolution of complex nervous systems was the differentiation in central ganglia plus nerve cord (central nervous system, CNS) and discrete peripheral nerves to connect the CNS with peripheral organs (Bullock and Horridge, 1965; Arendt et al., 2008). Neurons of the CNS and PNS evolved into highly specialized cells for precise and fast impulse propagation. Their processes, especially the axons commonly extend over long distances, which impedes the supply of distant neuronal domains. Therefore a second major step in nervous system evolution was the development of glial cells and their supporting association with neurons. As evolution progressed the ratio of glial cells to neurons increased (data for frontal cortex in Sherwood et al., 2006) such that pure numbers and complexity of neuroglia outnumbered the quantity of neurons by far (Oberheim et al., 2006; Reichenbach, 1989; Hartline, 2011, 2008). In the mammalian nervous system, we distinguish several different CNS and PNS (peripheral nervous system) glial cell types that took over discrete but partially overlapping tasks which neurons have abandoned or reduced in evolution. For example, the supply of nutrients and trophic factors is carried out by astrocytes and oligodendrocytes in the central nervous system as well as by Schwann cells in the peripheral nervous system. In addition, the removal of pathogens and dead cells is taken over by microglia (CNS) and Schwann cells (SC) and recruited macrophages in the PNS. Furthermore, astrocytes (CNS) and terminal SC (PNS) are involved in clearance of transmitters and modulation of synaptic transmission. Finally, the insulation of axonal segments, together with the ion homeostasis, are the best studied glial tasks which are taken over by oligodendrocytes in the CNS and Schwann cells in the PNS. Within the PNS mammals have additionally evolved glial cells that also ensheath neuronal cell bodies (satellite cells in the ganglia), olfactory ensheathing cells which are limited to the olfactory system and enteric ganglia, that are specifically associated with the enteric nervous system (summarized in Verkhratsky and Butt, 2013, Glial Physiology and Pathophysiology Chapter 3 Neuroglia; PNS glia reviewed in Jessen, 2004; Hanani, 2005; Corfas et al., 2004). As the work in this thesis is restricted to the analysis of peripheral nerves and their glia, the following will primarily focus on glial development and myelination in the PNS. 


\subsubsection{Evolution of myelin}

The bigger and more complex the organisms became, the greater was the demand for fast conduction, rapid reaction, metabolic efficiency, space economy together with stronger interconnection and more rapid information processing. Two opposing strategies, which are the increase of fiber size or the insulation of fibers, evolved in parallel to enhance conduction speed. In vertebrates as well as in some invertebrates insulation was achieved through development of multilayered myelin, an elaborated glial membrane that is wrapped around the axon and that contains only minimal amounts of electrical conductive cytoplasm in between the layers to optimize electrical insulation of enwrapped parts of the axons (illustrated in Fig I; reviewed in Hartline and Colman, 2007; Hartline, 2008). In higher vertebrates sheaths became compacted, which further raised resistance and elaborated compactness within the nervous system. The compaction of these specialized membranes, which contain about $70 \%$ lipids, especially cholesterol and glycosphingolipids (Norton and Autilio, 1965; Fu et al., 1998) is mediated by specific transmembrane (e.g. Myelin Protein zero, P0) and membrane associated proteins (e.g. myelin basic protein, MBP). Their mutations cause inappropriate myelination and compaction which is frequently associated with axonal loss (Giese et al., 1992; Readhead et al., 1987). Hence, the nearly fused compact myelin membranes in mammals appear as a layered structure with electron dense major dense lines (opposing intracellular sides of membranes) and interperiod lines (opposing extracellular membrane sides) (illustrated in Fig I). Additionally, the isolation simultaneously reduces the energy demands of neurons as the generation of action potentials and therefore ion fluxes are mainly restricted to specialized nodal areas (nodes of Ranvier in higher order vertebrates).

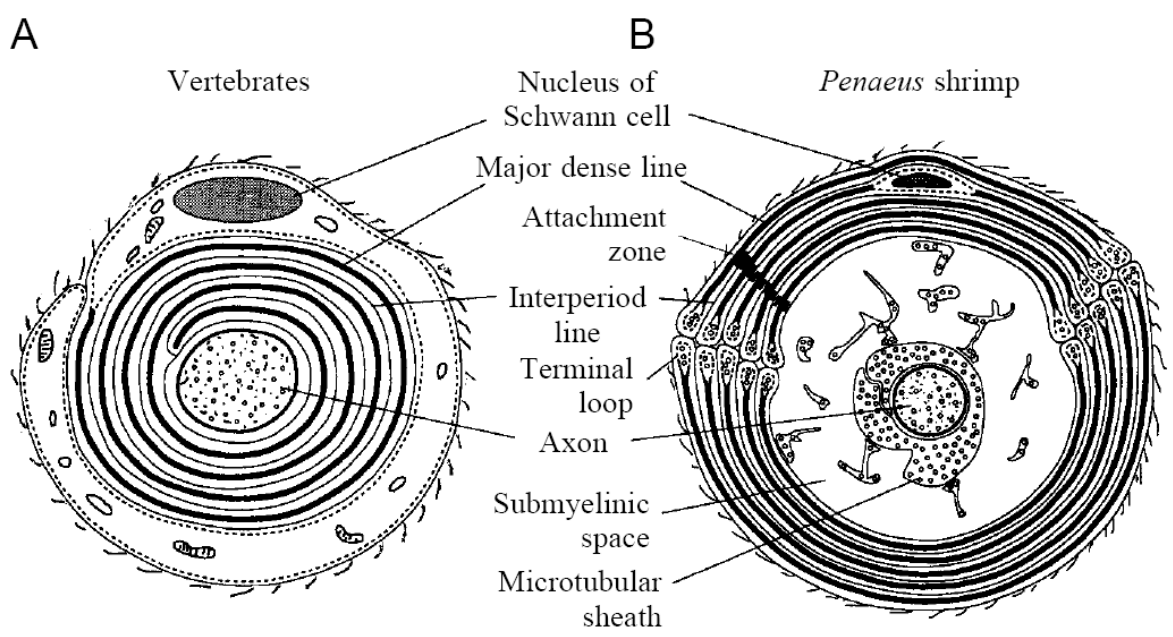

Fig I: Myelin structure in vertebrates and invertebrates

In vertebrates Schwann cells with a compacted spiraled myelin sheath (A) have evolved. In parallel, invertebrates as the Penaeus shrimp (B) developed a compact multilayered sheath with some noncompact areas and a submyelinic space. Both structures enormously increase conduction speed. (Adapted from Xu and Terakawa, 1999) 


\subsection{Schwann cells}

\subsubsection{The Schwann cell lineage}

In the PNS the isolation of nerve fibers is carried out by Schwann cells (SC). They originate from multipotent progenitors of the neural crest (Douarin et al., 1991) in early embryonic development. These cells migrate to the developing peripheral nerves and transform to Schwann cell precursors (SCP). Reaching their target, SCP, which are in contact with a group of axons, develop into immature Schwann cells that engulf the axons and form defined axon-Schwann cell bundles. Within the bundles fibers above $1 \mu \mathrm{m}$ in diameter are sorted out and gain a $1: 1$ relationship with a single Schwann cell that transforms into a promyelinating SC which will further mature and myelinate the engulfed internode. In contrast, axons smaller than $1 \mu \mathrm{m}$ in diameter remain grouped and engulfed by nonmyelinating Schwann cells to form mature Remak bundles (Fraher et al., 1990; Friede and Bischhausen, 1982; Pannese et al., 1987; Williams and Wendell-Smith, 1971). The SC development is illustrated in Fig II (Jessen and Mirsky, 2005, 2010).

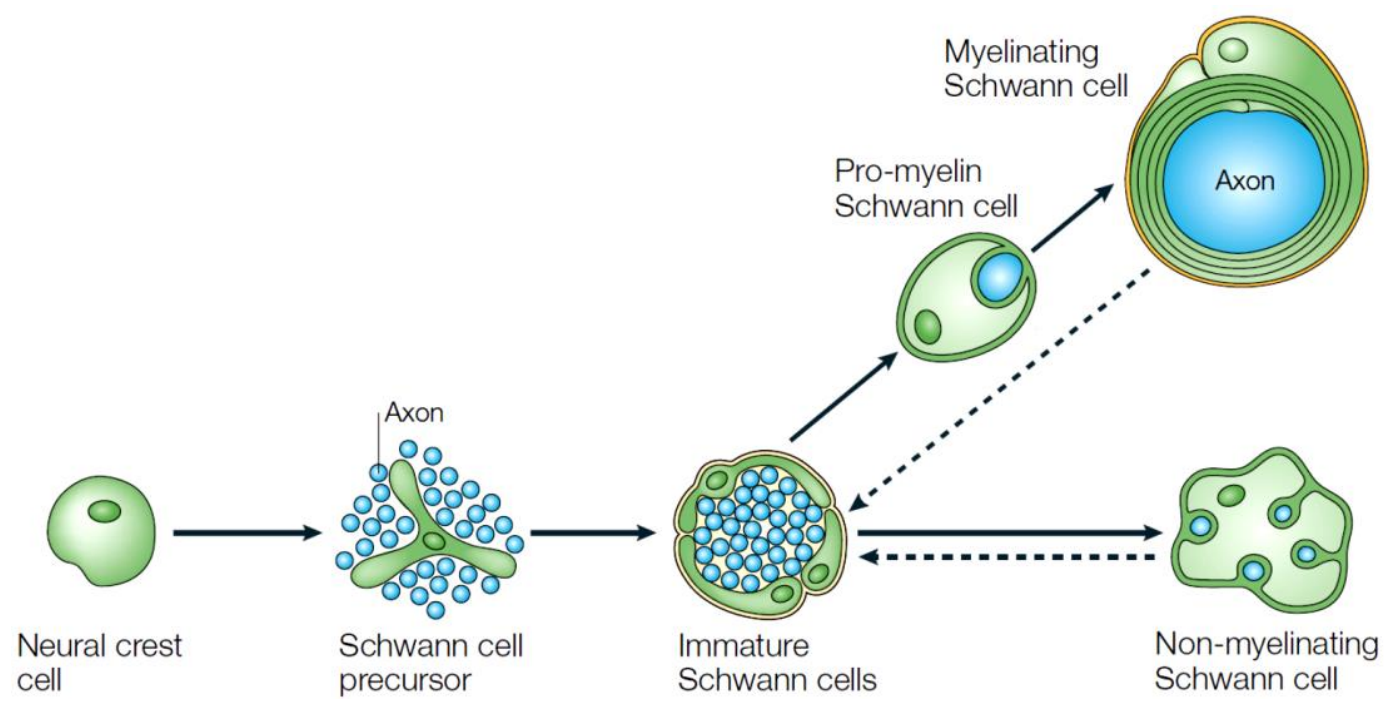

Fig II: The Schwann cell lineage

This drawing shows the Schwann cell lineage progression from migrating neural crest cells to Schwann cell precursors (SCP) that are in contact with groups of unsorted axons. SCP develop into immature SC that engulf groups of axons and radially sort big axons into $1: 1$ relationships to give rise to myelinating SC. Small axons are separated from each other by nonmyelinating SC but remain unmyelinated in Remak bundles. (Adapted from Jessen and Mirsky, 2005)

All phases and transitions are accompanied by individual marker expression. Transition from SCP to immature SC is irreversible, whereas mature Schwann cells can dedifferentiate to immature like repair cells (dashed lines in Fig II) upon peripheral nerve injury. 


\subsubsection{Regulation of Schwann cell development}

During early PNS development, it has been shown that growing axons and cells of the Schwann cell lineage are interdependent on each other (Morris et al., 1999; Garratt et al., 2000; Jessen and Mirsky, 2005). Insight came from observations that an ablation of the SC lineage specific trophic factor Sox10 or the ErbB3 receptor results in a lack of SCP and SC, which secondarily causes the degeneration of originally developed peripheral axons (Riethmacher et al., 1997; Britsch et al., 2001; Meyer and Birchmeier, 1995). On the other hand, the axonally expressed master regulator Neuregulin1 (Nrg1) inhibits neuronal development from neural crest cells (Shah et al., 1994) but promotes survival and proliferation of SCP and SC. This interdependence adjusts numbers of SC to axons (Birchmeier and Nave, 2008; Newbern and Birchmeier, 2010). From immature stages on, SC survival becomes independent of axons. Instead, they establish an autocrine survival loop which comprises the expression of growth factors like neurotrophin 3 (NT3), insulin-like growth factor 2 (IGF2), platelet-derived growth factor beta (PDGFB) and others (Jessen and Mirsky, 2005). This autocrine survival loop is pivotal for SC survival during peripheral nerve injury and regeneration. Although their survival is ensured, the fate of Schwann cells after the immature SC stage is determined by axonal derived signals such as Nrg1, IGF1 and the brain derived neurotrophic factor (BDNF). Those factors trigger radial sorting of large caliber axons and promote myelination initiation within the associated SC (Meintanis et al., 2001; Cheng et al., 2000; Taveggia et al., 2005). Promyelinating SC upregulate intrinsic feed forward signaling cascades involving Krox20, Oct6, Sox10 and NFkB expression but also continue to respond to cell-extrinsic trophic factors such as Nrg1, BDNF, IGF1 (Taveggia et al., 2005; Jessen and Mirsky, 2008). In early postnatal life promyelinating SC start to extend their plasma membrane up to one thousand fold within days so that rapid myelin growth can be observed (reviewed in Sherman and Brophy, 2005). As myelination precedes the compact myelin sheath is formed.

\subsubsection{Regulation of myelin sheath thickness}

Investigations in the early $20^{\text {th }}$ century revealed, that the myelin sheath thickness is proportional to the axonal diameter (Donaldson and Hoke, 1905) assuming that it is optimized for maximal nerve conduction velocities (Smith and Koles, 1970; Waxman, 1980). Furthermore, it was noted that not only the radial but also longitudinal dimension of the sheath is proportional to the size of the ensheathed axon defining a specific internodal length (Friede and Bischhausen, 1980, 1982). In the peripheral nervous system myelination begins at around birth and continues to adulthood (Jessen and Mirsky, 2005). As myelin grows, 
newly formed material is added at the innermost layer of the sheath at the adaxonal side. At the same time the sheath also expands longitudinally, thereby defining nodes of Ranvier. When development proceeds the cytoplasm between the myelin layers is removed and the sheath gets compacted from outside-in (referring to CNS myelination in Snaidero et al., 2014). Like in SC fate decision, myelin sheath thickness is also at least partially regulated by axonal Nrg1. Thus, axonal Nrg1-type III binds to ErbB receptors of the SC surface, which further integrate the axonal signals mainly via the MAPK/ERK pathways and/or PI3K/AKT pathway to promote lipid synthesis (Pertusa et al., 2007) and myelin protein expression (Newbern and Birchmeier, 2010; Michailov et al., 2004; Taveggia et al., 2005; Birchmeier and Nave, 2008). Disturbed axonal signaling can substantially impair myelination. For example, axonal Nrg1-type III can act bidirectionally as reduced levels result in hypomyelination, whereas on the other hand an increase in axonal Nrg1-type III causes a severe hypermyelination (Michailov et al., 2004). SC specific defects in the signaling pathways can provoke comparable effects. For instance the conditional ablation of ErbB2 (neuregulin receptor) in immature SC causes a striking hypomyelination (Garratt et al., 2000). Furthermore, defects in downstream signaling likewise affect myelination. Conditional inactivation of extracellular-signaling related kinases 1 and 2 (ERK1/2) in SC dramatically reduce myelination (Newbern et al., 2011). Opposing effects can be observed in dominant active Mek1DD mutants (constitutive active MAPK signaling) which display a hypermyelination by increased myelin protein and lipid synthesis (Ishii et al., 2013; Sheean et al., 2014). Interestingly, the inducible reactivation of Raf (upstream activator within the MAPK/ERK pathway) in mature myelinated SC induces demyelination and dedifferentiation (Napoli et al., 2012). Together these data impose a tight control of myelination with upregulation of promyelinating signals in early development and a coordinated downregulation as myelination reaches the appropriate myelin sheath thickness. Thus, when SC mature, the activity of the promyelinating PI3K/AKT and ERK/MAPK signaling pathways as well as the active translational machinery are downregulated to end myelination (Cotter et al., 2010; Sheean et al., 2014). Afterwards steady state levels of myelin protein and lipid production maintain the myelin sheaths throughout life. 


\subsubsection{Noncompact myelin and the axon-glia interaction}

The compact myelin sheath is optimized for electrical insulation to enable fast impulse propagation. Simultaneously it minimizes the access to external factors. Therefore a fundamental task of SC is the trophic and metabolic support of shielded axons to preserve axonal function and long-term PNS integrity. To this end, SC assemble a complex network of cytoplasm filled noncompact myelin regions that comprise Schmidt-Lanterman incisures (SLI) which are shortcuts through the compact myelin sheath, the inner tongue which is the actual axon-glia interface, the paranodal loops which are the lateral turns of the sheath and the inner as well as outer mesaxon, adjacent to first and last layers of the compact myelin (dark purple in Fig III). Together they form a network of transport routes, that are further connected by "autotypic" gap junctions and adherence junctions (Balice-Gordon et al., 1998; Tricaud et al., 2005; brown in Fig III). Presumably, these tracts facilitate the transport of myelin components themselves, as the sheath is growing at the inner mesaxon (shown for CNS myelin in Snaidero et al., 2014). Moreover, they allow the transport of metabolites for the most distal SC regions (innermost myelin layers and inner tongue) and the engulfed axon (via the axon-glia interface). Hence, pathological alterations within the noncompact myelin compartment may cause demyelination, triggered by insufficient transport of myelin components, or axonal degeneration by shortage of metabolites. The arrangement of the compartments is illustrated in Fig III.

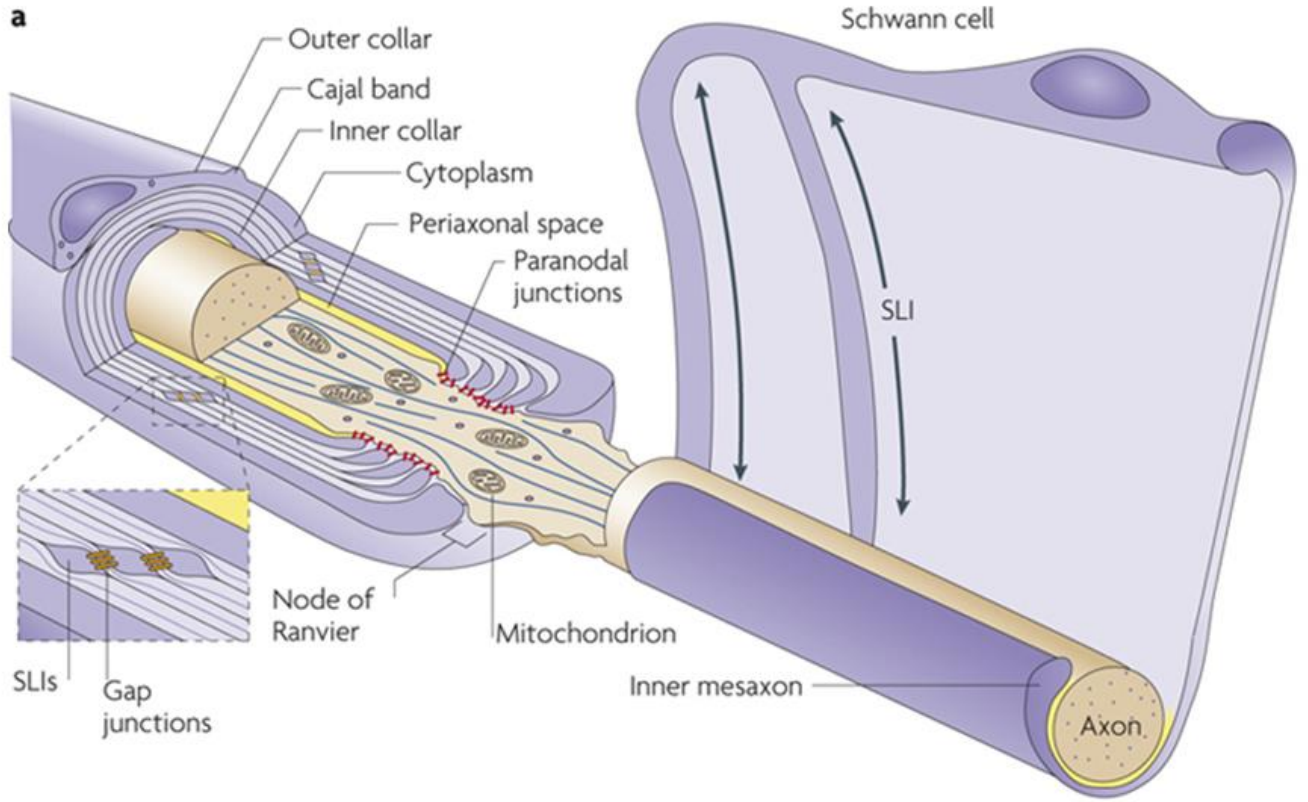

\section{Fig III: Scheme of a myelinated peripheral axon with unrolled sheath}

This scheme displays the organization and interconnections (gap junctions, brown) within the noncompact myelin compartment (dark purple) that comprises inner and outer mesaxons, Schmidt-Lanterman incisures (SLI) and paranodal loops. Noncompact myelin forms a transport network through the compact myelin sheath (light purple). (Adapted from Nave, 2010a) 
To underline the proposed involvement of noncompact myelin defects in the etiology of peripheral neuropathies, some glial defects which affect the conjuction and morphology of noncompact myelin are highlighted. For example impaired paranodal loop and SchmidtLanterman incisure morphology, as seen when the small integral membrane protein of late endosomes (SIMPLE) is mutated, contributes to peripheral nerve degeneration seen in CMT1C patients and according mouse models (Lee et al., 2013). Furthermore, derangements of noncompact myelin as detectable in MAG (myelin associated glycoprotein) mutants may play a fundamental role in pathological processes within peripheral nerves (Yin et al., 1998) which is also observable in aged mice and humans. Disruption of the innermyelinic transport has also been reported to cause axonal degeneration and demyelination as seen by several mutations of the gap junctions protein Connexin 32 (Cx32) typically found in men and mice with x-linked Charcot-Marie-Tooth disease (CMTX) (Scherer et al., 1995; Hahn et al., 2000, 2001). In addition to hampered innermyelinic transport processes, also decreased intercellular transfer along the axon-glia interface impairs nervous system integrity. For example, mice with an oligodendrocyte-specific reduction of the monocarboxylate transporter 1 (MCT1; likewise reduced in ALS patients) display axonal degeneration probably due to chronic undersupply of the engulfed internodal segments (Lee et al., 2012). Altogether, it is believed that the balance of compact and noncompact myelin as well as the efficient transport within the SC and across the SC-axon junction are necessary for long-term axonal survival and PNS integrity.

Substantial advances in Schwann cell research have been made in the investigation of the active involvement of SC in PNS homeostasis through direct axon-glia communication. There is increasing evidence that Schwann cells fuel peripheral axons, as oligodendrocytes and astrocytes do in the CNS (Pellerin et al., 1998; Fünfschilling et al., 2012; Lee et al., 2012). Peripheral nerve ex vivo studies by Vega and coworkers (2003) have shown that SC take up glucose which is most likely followed by a supply of lactate to the axon. A severe peripheral neuropathy in mice that exhibit non-functional mitochondria in SC (mitochondrial transcriptional factor A; Tfam; specifically ablated in SC), further manifested the proposed metabolic support to peripheral axons by SC (Viader et al., 2011). Confirmation of a direct metabolic coupling in the PNS came from on-demand breakdown of stored glycogen by myelinating SC to dynamically provide energy for active peripheral axons (Brown et al., 2012). Hence, SC not only give rise to the myelin sheath but also support the underlying axon. 


\subsubsection{Additional Schwann cell functions}

Apart from myelination and metabolic support Schwann cells are linked to many other processes crucial for peripheral nerve integrity. They are involved in the regulation of axonal diameter by an impact on neurofilament phosphorylation (Hsieh et al., 1994; Cole et al., 1994; Yin et al., 1998). Furthermore they promote ion channel clustering during development and maintenance of nodal morphology throughout life. Therefore, axonal and SC membranes at the nodal, paranodal and juxtaparanodal regions are interconnected with cell-adhesion molecules and adaptor proteins that simultaneously cluster and anchor sodium and potassium channels (Poliak and Peles, 2003). Schwann cells are furthermore immune competent cells as they are capable to express major histocompatibility complexes (MHCs) and other immunomodulatory proteins. Once SC recognize an antigen they start to attract immune cells. Additionally they are neuroprotective upon acute injury and can benefit peripheral nerve integrity during inflammatory processes (Armati et al., 1990; Armati and Pollard, 1996; Ydens et al., 2013). Another myelin independent feature of Schwann cells is their formation of a basal lamina and their ability to dedifferentiate and to form Büngner repair cells which become crucial in peripheral nerve repair after injury. When SC dedifferentiate, promyelinating programs (including myelin protein and lipid production) and positive myelin regulators are downregulated, whereas negative regulators of myelination (mostly active before myelination) will be reexpressed as seen for instance for c-Jun and Notch (Parkinson et al., 2008; Woodhoo et al., 2009). In order to promote axonal regeneration the dedifferentiated SC align to form tracts (bands of Büngner) and upregulate specific regeneration promoting ECM molecules such as laminins (Wallquist et al., 2002) which facilitate directed axonal regrowth. Simultaneously, dedifferentiated SC upregulate the expression of neurotrophic factors to promote axonal regrowth as reported for instance for Netrin 1 (Madison et al., 2000). In summary, SC take over diverse functions and are essential to maintain the PNS integrity in several independent ways. 


\subsubsection{Different myelinating Schwann cell types}

As described above, peripheral nerves are capable of regeneration. In several regeneration studies, especially in nerve graft experiments, a preferential regrowth of axons into their original tracts has been noted. Motor axons therefore show "preferential motor reinnervation" dependent on end organ presence (muscle) and SC characteristics (Höke et al., 2006; Madison et al., 2009). Similarly, sensory axons tend to reinnervate sensory tracts more efficiently (Höke et al., 2006). On molecular level denervated SC of motor and sensory nerves have been found to express "phenotype specific" genes like nerve growth factor (NGF), brain-derived growth factor (BDNF), vascular-endothelial growth factor (VEGF), insulin-like growth factor (IGF1) and hepatocyte growth factor (HGF) which are upregulated in sensory nerves, on the other hand the glial derived neurotrophic factor (GDNF) and pleiotrophin (PTN) are increased in motor nerve tracts (Höke et al., 2006; Jesuraj et al., 2012). These data suggest that SC have a "phenotypic memory" which is only lost during expansion in vitro or during prolonged periods of denervation. Moreover, it has been shown that recombinant overexpression of NGF indeed increases accuracy of sensory reinnervation (Hu et al., 2010). To broaden the spectrum further (Brushart et al., 2013) it has been reported that SC of different peripheral nerves in fact express distinct RNA profiles of the above mentioned growth factors, implying a variety of distinct myelinating SC types within the PNS. Apart from growth factors, cellular adhesion molecules are found in different abundances in denervated SC of motor and sensory tracts as shown for L2/HNK1 that is specifically expressed in SC formerly associated with motor axons (Martini et al., 1994). Nerve fibers on the other hand express specific neuronal cell adhesion molecule (NCAM) and polysialic acid (PSA), which interact with the ECM molecules of SC. Regrowing axons fail to show any preference for their appropriate tracts if these molecules are ablated (Franz et al., 2005). All together, it is evident that motor and sensory SC display differences in peripheral nerve regeneration. The phenotypic memory of SC, which is essential for proper reinnervation, on the other hand imposes, that SC may as well be different before a nerve injury. However, general differences within the population of myelinating SC apart from growth factors and adhesion molecules remain largely unknown. 


\subsection{2',3'-Cyclic Nucleotide 3'-Phosphodiesterase (CNP)}

Immense contribution to our understanding of myelin functionality has been made by the analysis of single myelin proteins. One of those well studied myelin proteins is CNP. In the 1960s a new enzyme, specifically catalyzing the hydrolysis of 3'-phosphoester bonds in 2',3'cyclic nucleotides, was discovered in the intestinal mucosa. Due to its catalyzed reaction it was named 2',3'-cyclic nucleotide 3'-phosphodiesterase - CNP (Whitfeld et al., 1955). Later this protein was found to be enriched in the brain that as well yielded catalytically active CNP (Drummond and Perrott-Yee, 1961; Drummond et al., 1962). Refined investigations have further restricted CNP to white matter regions of the brain as well as peripheral nerves. Here, its activity was specifically linked to the myelin sheath, which was 10 times stronger in the CNS compared to PNS samples (Kurihara and Tsukada, 1967). Within myelinating oligodendrocytes and SC, CNP was found on the cytoplasmic side of the plasma membrane in all noncompact myelin regions (Braun et al., 1988; Trapp et al., 1988). CNP expression can be found already in the oligodendrocyte and SC lineage in embryonic stages and is therefore one of the earliest markers of myelinating glial cells (Sprinkle et al., 1978a; Scherer et al., 1994).

\subsubsection{Molecular characteristics of CNP}

The CNP gene is well conserved and shares $96 \%$ sequence homology between rat and mouse and $86 \%$ homology between rat and human (Gravel et al., 1994). Due to two alternative promoters, the CNP gene gives rise to two mRNAs and protein isoforms (46 kDa and $48 \mathrm{kDa}$, additional N-terminal mitochondrial localization sequence) (Bernier et al., 1987; Kurihara et al., 1990; Lee et al., 2005). Whereas oligodendrocytes and SC generate both mRNAs (mainly CNP1) and express both proteins, non-glial cells outside the nervous system only express the CNP2-mRNA and the mitochondrial linked CNP2 protein. Interestingly, the shorter CNP2-mRNA contains both promoter regions and can therefore give rise to both protein isoforms. Within the nervous system, glial precursor cells also mainly express the mitochondrial CNP2, whereas in active myelination phases the CNP1 isoform, which is localized to noncompact myelin is additionally upregulated (Scherer et al., 1994; O'Neill et al., 1997). The transcriptional regulation of CNP1 and CNP2 expression is not yet well understood. Insight came from in vitro observations that showed that enhanced cAMP levels together with a putative activator can induce especially CNP1 transcription likely resembling processes during development and active myelination (Gravel et al., 2000). 


\subsubsection{Catalytic activity of CNP}

Originally CNP was identified due to its unique site-specific catalytic activity. Ever since, the importance of the catalytic function of CNP has been under debate as there was lack of evidence for a physiological substrate within the nervous system. However, the catalytic domain of CNP was mapped to the C-terminus, which is further reported to be highly conserved among vertebrates (Lee et al., 2001). As previously mentioned, the CNP2 isoform is associated with mitochondria, except when the mitochondrial localization signal is phosphorylated, which prevents its translocation (Lee et al., 2006). Mitochondrial CNP seems to be catalytically active and thus influences 2',3'-cyclic nucleotide levels, which trigger the $\mathrm{Ca}^{2+}$-dependent permeability transition pore opening in mitochondria of apoptotic cells or in disease (Azarashvili et al., 2009, 2010, 2014). Here, 2',3'-cAMP and 2',3'-cNADP have been suggested as possible substrates. 2',3'-cAMP originates from RNA processing and could be hydrolyzed to neuroprotective adenosine (Thompson et al., 1994; Ren et al., 2009; Jackson et al., 2009). Indeed, the CNS has been shown to express the extracellular 2',3'-cAMP - adenosine pathway. Therein, CNP efficiently hydrolyses 2',3'-cAMP to 2'-AMP which is further degraded to adenosine. This reaction cycle, which is mainly carried out by oligodendrocytes, is highly efficient compared to the production of adenosine from 3',5'cAMP within the brain. This prevents the cytotoxic accumulation of 2',3'-cAMP and simultaneously provides neuroprotective adenosine locally. This pathway is strongly induced post injury but additional mutation of CNP worsens the outcome of experimental brain injuries (Stone et al., 2009; Verrier et al., 2012, 2013). SC also express adenosine receptors (Stevens et al., 2004), we therefore hypothesize that the extracellular 2',3'-cAMP-adenosine pathway may also play a role in the PNS as described above for the CNS.

\subsubsection{CNP functions independent of the catalytic activity}

Numerous other observations that may not be related to enzymatic function further propose CNP as structural protein essential for cell dynamics and morphology. It has been proposed that CNP is a cytoskeleton associated protein. Hence, it preferentially binds to tubulin heterodimers and induces microtubule assembly as well as the stabilization of the tubulin cytoskeleton. Accordingly, in cultured oligodendrocytes it promotes process outgrowth, which was elaborated when CNP was overexpressed (Lee et al., 2005). Importantly, deletion of the C-terminal domain (catalytic region) of CNP interferes with the microtubule stability. Along with the impact on the tubulin cytoskeleton, CNP has further been shown to bind to membranes in an isoprenylation-dependent manner. Therefore it may serve as an adjustable linker of cytoskeleton and the adjacent plasma membranes (Braun et al., 1991; Bifulco et al., 
2002). Moreover, CNP is regarded as a linker of RNA granules to the cytoskeleton as CNP can furthermore directly bind to RNAs and prevent active translation by interfering with the formation of the translation initiation complex. This translational repression may be a fundamental role of CNP in coordinated MBP-mRNA-granule transport within glial cells (Gravel et al., 2009). Together, these observations in combination with the potential catalytic function propose versatile roles for CNP with regard to cellular integrity.

\subsubsection{Overexpression and deficiency of CNP}

To further gain insight into the physiological role of CNP within the nervous system, several mouse models that harbour CNP mutations have been established. Initially, overexpression of human CNP revealed an involvement of CNP in myelination regulation. Thus, a shift towards an earlier peak of the myelin gene expression indicated an accelerated maturation of oligodendrocytes. Ultrastructurally, these mice exhibit redundant myelin, myelin vacuoles and widespread deficits in myelin compaction (lack of MDL). That was at least partially explained by defective MBP-mRNA pathfinding or spatial competition (Gravel et al., 1996; Yin et al., 1997). Thereafter, CNP deficient mice have been engineered by replacing the CNP open reading frame by the Cre-recombinase sequence. Homozygous mice are viable and inconspicuous in development and juvenile stages but start to develop a phenotype at four months, which deteriorates with age leading to premature death at around 9-11 months. Examining their central nervous system a striking neurodegeneration with pronounced white matter atrophy and astrogliosis as well as microgliosis can be observed. Interestingly, the CNS myelin ultrastructure was reported to be normal (Lappe-Siefke et al., 2003). Later, refined investigations of their spinal cords and optic nerves uncovered degeneration of small caliber fibers as early as postnatal day 5 . Additionally abnormally enlarged inner tongues as well as the accumulation of myelin spheroids have been observed throughout life (Edgar et al., 2009). Moreover, a progressive disorganization of nodal structures in CNP deficient optic nerves occurs even before the onset of axonal degeneration (Rasband et al., 2005). Together, the pathological alterations indicate a role of CNP in regulation and maintenance of noncompact myelin structures that furthermore have an impact on the compact myelin sheath. Recently published ultrastructural observations of fastened compaction within the developing CNS of CNP deficient mice (Snaidero et al., 2014) confirmed the importance of CNP as myelin regulator and structural coordinator. So far, the role of CNP in SC and its importance for PNS integrity had not been analyzed. 


\subsubsection{CNP in disease}

Peripheral neuropathies are commonly accompanied by demyelination and thus reduced myelin protein expression. Decreased CNP levels have early been reported for mouse mutants with a defective myelin sheath for instance tremblerJ (PMP22 point mutation; Lewis et al., 1984) and twitcher mice (GalC deficiency; Mikoshiba et al., 1985). It remained elusive if reduced CNP levels are directly involved in disease etiology. Furthermore, a potential involvement of CNP function in nervous system degeneration and regeneration has been suggested by LeBlanc and coworkers (1992) who observed decreased CNPase activity during degeneration phases in experimental peripheral neuropathies followed by an increase in activity during regeneration. CNP was also connected to several human diseases for instance schizophrenia (Peirce et al., 2006) as the CNP gene lies within a chromosomal region that is linked to the disease. Moreover, the brains of schizophrenic patients frequently show reduced CNP levels. This can be nicely resembled by aged CNP heterozygous mice, that also show a catatonia-depression syndrome, a schizophrenia-associated disorder (Hagemeyer et al., 2012). Additionally, decreased levels of CNP have further been observed in patients with Down syndrome or Alzheimer's disease (Vlkolinský et al., 2001). Moreover, multiple sclerosis patients frequently show elevated CNP levels in their cerebrospinal fluid (Sprinkle et al., 1978b; Suda et al., 1984). More recently, CNP has been shown to have Tcell-attractive properties and may therefore directly influence development of multiple sclerosis (Muraro et al., 2002). Together, these data suggest a role of CNP in a variety of diseases possibly referring to a final common pathway in degeneration of central white matter. Surprisingly, the connection to human disease has only been shown for reduced CNP levels but no loss- or gain-of-function mutations have been reported to date. However, this work will provide insight into pathological alterations within the PNS of CNP deficient mice. 


\subsection{Aim of the study}

Motor and sensory SC of injured peripheral nerves differ with regard to the expression of regeneration promoting factors to specifically promote the regrowth of appropriate axons. The "phenotypic memory" implies that differences between the two SC types are established in development and can be observed also in uninjured nerves. We hypothesized, that specific characteristics of motor and sensory SC not only comprise extracellular matrix components and trophic factors but potentially involve basic differences in the myelin constitution, as SC are associated with distinct axonal subtypes that likewise display different features. In this work the postulated myelin sheath diversity was addressed by comparative proteome analyses and quantitative proteinbiochemical investigations of purified myelin from motor and sensory nerves. Finding variability in their myelin composition, the question about the differential importance of single myelin proteins for discrete fiber types arose. Having found, that the noncompact myelin protein CNP is indeed differentially expressed, the second and major part of this work addressed the role of CNP function within the PNS. Initial investigations focused on the question of whether CNP, which is ten times less abundant in the PNS compared to the CNS, has a similar impact on SC functionality and peripheral nerve integrity as it was shown for oligodendrocytes in the CNS. To this end, peripheral nerves of CNP deficient mice were compared to nerves of wildtype animals. Histological and electron microscopic analyses aimed to uncover morphological alterations particularly in SC but also on the axonal side. Special emphasis was placed on the differentiation of changes within the noncompact and compact myelin compartment. Furthermore, analyses of gene regulation and activity of signaling pathways provided insight in underlying pathomechanisms. In relation to the differential protein expression between SC types, CNP is a good candidate to investigate the particular importance of myelin proteins for discrete fiber populations. Therefore, refined morphological and biochemical analyses of different peripheral nerves emphasized the specific impact of CNP function on large and small fiber integrity in the PNS. This work aimed to combine the analysis of a general SC diversity with respect to their myelin sheaths, and the detailed investigation of one differentially expressed protein (CNP) with special emphasis to its particular importance for the integrity of discrete peripheral nerve fiber subpopulations. 


\section{Materials}

\subsection{Chemicals and consumables}

Except otherwise stated all chemicals and consumables are purchased from Sigma-Aldrich, Merck, Serva, Falcon, Eppendorf and Invitrogen.

\section{$\underline{\text { General }}$}

BSA

Ketamine, $10 \%$

Low fat milk powder

Rompun, $2 \%$
BioMol

CP-Pharma

Frema

Bayer

Merck

Kindler / Science Services

Plano

Electron Microscopy Science (EMS)

Serva

Merck

Serva

Merck

Science Services

Serva

Sigma-Aldrich

Science Services

SPI-Chem

\section{$\underline{\text { Protein biochemistry }}$}

Acrylamide

Serva

Ammonium persulfate (APS)

BioRad

Bromphenol blue

Sigma

Complete Mini (Protease inhibitors)

Roche

Formaldehyde $37 \%$

Merck

Glycine

Sigma

2-Mercaptoethanol

Fluka

PhosStop (Phosphatase inhibitors)

Roche 
Prestained Page Ruler Plus

SDS (Dodecylsulfate sodium salt)

TEMED

Trizma Base

Tween20 (Polysorbate)

Molecular biology

Agarose

GelRed

GenRuler 1 kb DNA ladder

GenRuler 100 bp DNA ladder

GoTaq polymerase

Power SybrGreen

Trizol Reagent

Superscript III-reverse Transcriptase

$\underline{\text { Immunohistochemisty }}$

Aqua Polymount

Goat serum

Haematoxylin (hemalum solution)

Horse serum

Paraformaldehyde, pure

\section{$\underline{\text { Consumables }}$}

Copper grids (100Mesh, Guitarr, Slot)

Coverslips

Histobond slides

PVDF Western Blotting membranes

Superfrost Plus slides

$\underline{\text { Kits }}$

Agilent RNA 6000 Nano Kit

DAB Substrate Kit

DC Protein Assay Kit

Genomic DNA-Isolation Kit

GoTaq DNA polymerase

RNeasy Mini / Micro Kit
Fermentas

Serva

Serva

Sigma

Merck

AppliChem

Biotrend

Fermentas

Fermentas

Promega

Applied Biosystems

Invitrogen

Invitrogen

Polysciences

Gibco

Merck

Gibco

Serva

Gilder Grids

Thermo Scientific Menzel-Gläser

Marienfeld

Roche

Thermo Scientific Menzel-Gläser

Agilent

Zytomed

BioRad

Nexttec

Promega

Qiagen 
SuperScript III Reverse Transcriptase

Vectastain Elite ABC Kit

Western Lightning Plus ECL

\section{Machines}

AFS II

Agilent 2100 Bioanalyzer

Axiophot Observer inverted

Centrifuge 5415R

Diamond knife Histo (45\%/Ultra $45^{\circ}$ )

Embedding center AP280-1/2/3

EMTP (Epon infiltration)

EM900

EM912AB

HPM100

Intas ChemoStar

Light Cycler 480

NanoDrop200 Spectraphotometer

Optima TLX Ultracentrifuge

Precellyse24

PCR-machine

Sled microtome

Thermomax microplate reader

Tissue Processor HMP110

Ultracut

UV illuminator

Wet Chamber Blotting System

7500 Fast Real-Time PCR System
Invitrogen

Vector

Perkin Elmer Life Sciences

Leica

Agilent

Zeiss

Eppendorf

Diatome

Microm

Lynx

Zeiss

Zeiss

Leica

Intas

Roche

Thermo Scientific

Beckman

Bertin Technologies

PeqLab

Microm

MGW Biotech

Microm

Leica

Intas

BioRad

Applied Biosystems 


\subsection{Solutions and buffers}

\section{Avertin}

$2 \%[\mathrm{w} / \mathrm{v}] \quad$ 2,2,2-Tribrome ethanol 99\%

$2 \%[\mathrm{v} / \mathrm{v}] \quad$ Amyl alcohol

Mix at $40^{\circ} \mathrm{C}$ for $30 \mathrm{~min}$ while stirring. To be filtered before storage at $-20^{\circ} \mathrm{C}$.

\section{Anesthetic}

$4 \mathrm{mg} \quad$ Xylazine (2\% Rompun, BayerVital)

$60 \mathrm{mg} \quad$ Ketamine (10\% Ketamin, WDT)

Intraperitoneal injection of $6 \mathrm{mg}$ Ketamine and $90 \mathrm{mg}$ Xylazine per $\mathrm{kg}$ of bodyweight per mouse.

\section{1x Phosphate buffered saline (PBS)}

$170 \mathrm{mM} \quad$ Sodium chloride

3,4 mM Potassium chloride

$4 \mathrm{mM}$ di-Sodium hydrogen phosphate

1,8 $\mathrm{mM}$ di-Potassium hydrogene phosphate

Adjust to $\mathrm{pH} 7.4$ with $1 \mathrm{~N} \mathrm{NaOH}$.

\subsection{Phosphate buffer (working solution)}

$0,2 \mathrm{M} \quad \mathrm{NaH}_{2} \mathrm{PO}_{4}$ (Stock solution)

$0,2 \mathrm{M} \quad \mathrm{Na}_{2} \mathrm{HPO}_{4}$ (Stock solution)

Mix in $1: 5$ ratio and add equal amount of $d_{d d} \mathrm{H}_{2} \mathrm{O}$.

\section{Fixatives}

16\% Paraformaldehyde (PFA stock solution)

$16 \%[\mathrm{w} / \mathrm{v}] \quad$ Paraformaldehyde

Stir for $20 \mathrm{~min}$ at $60^{\circ} \mathrm{C}$ and subsequently increase $\mathrm{pH}$ with $\mathrm{NaOH}$ to allow full dissolution. Store at $-20^{\circ} \mathrm{C}$.

\section{4\% PFA in PBS (Fixative)}

$4 \%[\mathrm{v} / \mathrm{v}] \quad$ Paraformaldehyde

$0,1 \mathrm{M} \quad$ Phosphate buffer

$0,8 \%[\mathrm{w} / \mathrm{v}] \quad$ Sodium chloride

Adjust $\mathrm{pH}$ to 7.4 before use. 
K\&S fixative (according to Schultz and Karlsson, 1965)

$25 \%[v / v] \quad 16 \%$ Paraformaldehyde

$2,5 \%[\mathrm{v} / \mathrm{v}] \quad$ Glutardialdehyde

$50 \%[\mathrm{v} / \mathrm{v}] \quad 0.2 \mathrm{M}$ phosphate buffer

$0,08 \mathrm{M} \quad$ Sodium chloride

Adjust $\mathrm{pH}$ to 7.4 before use.

Molecular biology

dNTPs (50x stock solution)

$25 \mathrm{mM}$ of dATP, dGTP, dCTP, dTTP (Boehringer, Mannheim)

TBE 20x (stock solution)

$1.8 \mathrm{M} \quad$ TrisBase

$1.8 \mathrm{M} \quad$ Boric acid

$200 \mathrm{mM} \quad$ EDTA

$\underline{\text { Protein biochemical solutions }}$

\section{Sucrose buffer (Homogenisation)}

$320 \mathrm{mM} \quad$ Sucrose

$10 \mathrm{mM} \quad$ Tris- $\mathrm{HCl} \mathrm{pH} 7.4$

$1 \mathrm{mM} \quad$ Sodium hydrogen carbonate

$1 \mathrm{mM} \quad$ Magnesium chloride

Add proteinase inhibitors and optionally phosphatase inhibitors before use.

\section{Running buffer (Laemmli buffer working solution)}

$25 \mathrm{mM} \quad$ TrisBase

$192 \mathrm{mM} \quad$ Glycine

$1 \%[\mathrm{w} / \mathrm{v}] \quad$ SDS

Transfer buffer (1x for wet chamber blotting)

$48 \mathrm{mM} \quad$ TrisBase

$39 \mathrm{mM} \quad$ Glycine

$20 \%[v / v] \quad$ Methanol 
Tris buffered saline with Tween20 (TBST)

$50 \mathrm{mM} \quad$ Tris- $\mathrm{HCl}(\mathrm{pH} \mathrm{7.4)}$

$150 \mathrm{mM} \quad$ Sodium chloride

$0.05 \%[\mathrm{v} / \mathrm{v}] \quad$ Tween20

Protein loading dye (4x)

40\% [w/v] Glycine

$240 \mathrm{mM} \quad$ Tris- $\mathrm{HCl} \mathrm{pH} 6.8$

$8 \%[\mathrm{w} / \mathrm{v}] \quad$ SDS

0,04\% [w/v] Bromphenole blue

SDS separation gel

10-16\% [v/v] Acrylamide / Bisacrylamide 29:1

$0,4 \mathrm{M} \quad$ Tris- $\mathrm{HCl} \mathrm{pH} 8.8$

$0,1 \%[\mathrm{w} / \mathrm{v}] \quad$ SDS

$0,03 \%[\mathrm{v} / \mathrm{v}] \quad$ Ammonium persulfate

$0,08 \%[\mathrm{v} / \mathrm{v}] \quad$ TEMED

SDS stacking gel

$4 \%[\mathrm{v} / \mathrm{v}] \quad$ Acrylamide / Bisacrylamide 29:1

$125 \mathrm{mM} \quad$ Tris- $\mathrm{HCl} \mathrm{pH} 6.8$

$0,1 \%[\mathrm{w} / \mathrm{v}] \quad$ SDS

0,05\% [w/v] Ammonium persulfate

$0,1 \%[\mathrm{w} / \mathrm{v}] \quad$ TEMED

Sucrose solution for myelin purification

$\begin{array}{ll}0.29 \mathrm{M} & \text { Sucrose } \\ 0.85 \mathrm{M} & \text { Sucrose }\end{array}$

Add protease inhibitors to the solutions.

Gel fixative (for silver and coomassie stainings)

$40 \%[\mathrm{v} / \mathrm{v}] \quad$ Ethanol

$10 \%[\mathrm{v} / \mathrm{v}] \quad$ Acetic acid 


\section{Solutions for electron microscopy}

Reynolds lead citrate solutions (Reynolds, 1963)

$1.33 \mathrm{~g} \quad$ Lead nitrate

$1.76 \mathrm{~g} \quad$ Sodium citrate

Solve in $30 \mathrm{ml} \mathrm{ddH_{2 }} \mathrm{O}$ while shaking. After 30 min add $8 \mathrm{ml}$ of $1 \mathrm{M} \mathrm{NaOH}$ to adjust the $\mathrm{pH}$ to 12. Add $\mathrm{ddH}_{2} \mathrm{O}$ to $50 \mathrm{ml}$. Sterile filter before use.

\section{Uranyl acetate}

$4 \%[\mathrm{w} / \mathrm{v}] \quad$ Uranyl acetate

Aqueous solution has to be filtered before use.

\section{Epoxy resin (Epon)}

$21.4 \mathrm{~g} \quad$ Glycid ether 100

$14.4 \mathrm{~g} \quad$ DDSA (Serva, 20755)

$11.3 \mathrm{~g} \quad$ MNA (Serva, 29452)

Stir for $10 \mathrm{~min}$ and subsequently add $0.84 \mathrm{ml}$ DMP-30. Stir for another $20 \mathrm{~min}$.

\section{Formvar solution}

$1.25 \%[\mathrm{w} / \mathrm{v}] \quad$ Formvar

$50 \mathrm{ml} \quad$ Chloroform

Stir for at least $30 \mathrm{~min}$. Protect from light. Store at room temperature.

Richardson's Methylene blue / Azure II blue (Richardson et al., 1960)

$1 \%[\mathrm{w} / \mathrm{v}] \quad$ Azure II in $\mathrm{dd}_{2} \mathrm{O}$ (Stock solution 1 )

$1 \%[\mathrm{w} / \mathrm{v}] \quad$ Methylene Blue in 1\% Borax (Sodium borate) (Stock solution 2)

Mix equal amount of stock solutions and filter before use.

\section{$\underline{\text { Histological staining solutions }}$}

Citrate buffer (0.01 M, pH 6.0)

$1.8 \mathrm{mM} \quad$ Citric acid

$8.2 \mathrm{mM} \quad$ Sodium citrate

Always prepare freshly and adjust $\mathrm{pH}$ to 6.0 .

Tris buffer (50 mM, pH 7.6)

$50 \mathrm{mM} \quad$ Tris- $\mathrm{HCl} \mathrm{pH} 7.6$

$0.9 \%[\mathrm{w} / \mathrm{v}] \quad$ Sodium chloride

Prepare freshly and adjust $\mathrm{pH}$ to 7.6. 


\section{Tris buffered milk}

$50 \mathrm{mM} \quad$ Tris- $\mathrm{HCl} \mathrm{pH} 7.6$

$0.9 \%[\mathrm{w} / \mathrm{v}] \quad$ Sodium chloride

$2 \%[w / v] \quad$ Low fat milk powder

Stir for $20 \mathrm{~min}$ at room temperature and filter before use.

\section{Blocking solution (PBS / BSA)}

$40 \mathrm{mM} \quad$ Sodium dihydrogen phosphate

$160 \mathrm{mM} \quad$ Disodium hydrogen phosphate

$1.8 \%[\mathrm{w} / \mathrm{v}] \quad$ Sodium chloride

$1.0 \%[\mathrm{w} / \mathrm{v}] \quad$ Bovine serum albumin

$1.0 \%[\mathrm{w} / \mathrm{v}] \quad$ Goat serum

\section{Blocking buffer for teased fiber stainings}

$\begin{array}{ll}10 \%[\mathrm{v} / \mathrm{v}] & \text { Horse serum } \\ 0.1 \%[\mathrm{v} / \mathrm{v}] & \text { Tween20 }\end{array}$

Add freshly to phosphate buffered saline (1x PBS).

\section{Mayer's haematoxylin solution (nuclear stain)}

$0.1 \%[\mathrm{w} / \mathrm{v}] \quad$ Hematoxylin

$0.02 \%[\mathrm{w} / \mathrm{v}] \quad$ Sodium iodate

$5 \%[\mathrm{w} / \mathrm{v}] \quad$ Potassium aluminium sulfate

Add following substances while shaking.

$5 \%[\mathrm{w} / \mathrm{v}] \quad$ Chloral hydrate

$0.1 \%[\mathrm{w} / \mathrm{v}] \quad$ Citric acid

Solution turns violet blue. Has to be filtered before use.

\section{HCl-Alcohol}

$0.09 \%[\mathrm{v} / \mathrm{v}] \quad$ Hydrogen chloride fuming

$70 \%[\mathrm{v} / \mathrm{v}] \quad$ Ethanol

\section{Scott's solution}

$0.2 \%[\mathrm{w} / \mathrm{v}] \quad$ Potassium hydrogen carbonate

$2 \%[\mathrm{w} / \mathrm{v}] \quad$ Magnesium sulfate 


\subsection{Antibodies}

\section{Primary antibodies}

\begin{tabular}{|c|c|c|c|}
\hline Actin & Sigma \#A3853 & mouse & $1: 5000$ \\
\hline AKT & Cell signaling \#4691 & rabbit & $1: 1000$ \\
\hline $\mathrm{p}-\mathrm{AKT}$ & Cell signaling \#3787 & rabbit & $1: 1000$ \\
\hline Caspr & Neuromab (\#P97846) & mouse & $1: 750$ (WB) / $1: 200(\mathrm{IHC})$ \\
\hline CD3 & Serotec (\#MCA1477) & rat & $1: 150(\mathrm{IHC})$ \\
\hline CNPase & Sigma \#C5922 & mouse & $1: 5000(\mathrm{WB}) / 1: 150(\mathrm{IHC})$ \\
\hline Cx29 & Life Tech \#34-4200 & rabbit & $1: 500$ \\
\hline DAPI & Roche (\#10236276001) & all DNA & $1: 20000(\mathrm{IHC})$ \\
\hline DM20/PLP (A431) & selfmade Dept. Nave & rabbit & $1: 5000(\mathrm{WB})$ \\
\hline GAPDH & Stressgene \#CSA-335 & mouse & $1: 5000$ \\
\hline MAC3 & BP-Pharmingen (\#553322) & rat & $1: 400(\mathrm{IHC})$ \\
\hline MAG & Chemicon \#MAB1567 & mouse & $1: 500(\mathrm{WB}) / 1: 50(\mathrm{IHC})$ \\
\hline MAPK & Cell signaling \#4695 & rabbit & $1: 1000$ \\
\hline p-MAPK & Cell signaling \#9101 & rabbit & $1: 1000$ \\
\hline MBP & Dako \#A0623 & rabbit & $1: 5000(\mathrm{WB}) / 1: 500(\mathrm{IHC})$ \\
\hline MBP & Serotec (\#MCA409S) & rat & $1: 400(\mathrm{IHC})$ \\
\hline MyP2 & Santa Cruz \#49304 & goat & $1: 200(\mathrm{WB})$ \\
\hline Nav1.6 & Alomone Labs (\#ASC009) & rabbit & $1: 500(\mathrm{IHC})$ \\
\hline Peripherin & Millipore (\#AB1530) & rabbit & $1: 400(\mathrm{IHC})$ \\
\hline Sept2 & PTG \#11397 & rabbit & $1: 500$ \\
\hline Sirt2 & Southwood et al., 2006) & rabbit & $1: 2000$ \\
\hline Tuj1 (tubulin beta III) & Covance (\#PRB-435P) & rabbit & $1: 400(\mathrm{IHC})$ \\
\hline
\end{tabular}

\section{$\underline{\text { Secondary antibodies }}$}

$\begin{array}{llll}\text { Mouse-HRP } & \text { Dianova }(\# 115-035-003) & \text { goat } & 1: 5000(\mathrm{WB}) \\ \text { Rabbit-HRP } & \text { Dianova }(\# 111-035-003) & \text { goat } & 1: 5000(\mathrm{WB}) \\ \text { Goat-HRP } & \text { Dianova }(\# 705-035-003) & \text { donkey } & 1: 5000(\mathrm{WB}) \\ \text { Mouse-A488 } & \text { Invitrogen (\#A21202) } & \text { donkey } & 1: 2000(\mathrm{IHC}) \\ \text { Mouse-A555 } & \text { Invitrogen (\#A31570) } & \text { donkey } & 1: 2000(\mathrm{IHC}) \\ \text { Rabbit-A488 } & \text { Dianova (\#711-545-152) } & \text { donkey } & 1: 2000(\mathrm{IHC}) \\ \text { Rabbit-A555 } & \text { Dianova (\#111-545-144) } & \text { donkey } & 1: 2000(\mathrm{IHC}) \\ \text { Rat-Dyeligh633 } & \text { YoProteins (\#309) } & \text { donkey } & 1: 500(\mathrm{IHC}) \\ \text { Rat-Biotin } & \text { Vector (\#BA-9400) } & \text { rabbit } & 1: 1000(\mathrm{IHC})\end{array}$




\subsection{Primers}

All oligonucleotides were synthesized by the AGCT-laboratory of the Max Planck Institute for Experimental Medicine.

\section{$\underline{\text { CNCE genotyping primer }}$}

CNP fwd: GCCTTCAAACTGTCCATCTC (Exon3 upstream of Bgl-II side) (\#2016)

CNP rev: CCCAGCCCTTTTATTACCAC (Exon3 downstream of Bgl-II side) (\#7315)

Cre fwd: CCTGGAAAATGCTTCTGTCCG (sense in Cre sequence) (\#4193)

Cre rev: CAGGGTGTTATAAGCAATCCC (antisense in Cre sequence) (\#4192)

\section{qRT-PCR primer}

bACT fwd: CGCTCAGGAGGAGCAATG rev: TGACAGGATGCAGAAGGAGA

CNP fwd: CGCTGGGGCAGAAGAATA rev: AAGGCCTTGCCATACGATCT

CycPh fwd: CACAAACGGTTCCCAGTTTT rev: TTCCCAAAGACCACATGCTT

HmgCR fwd: CAACCTTCTACCTCAGCAAGC rev: CACAGTGCCACATACAATTCG

MAG fwd: TCTACCCGGGATTGTCACTG rev: GCAGCCTCCTCTCAGATCC

MBP fwd: GCCTGTCCCTCAGCAGATT rev: GCCTCCGTAGCCAAATCC

PO fwd: TGCCCTGCTCTTCTCTTCTT rev: CCTGTCCGTGTAAACCACAA

PLP fwd: TCAGTCTATTGCCTTCCCTAGC rev: AGCATTCCATGGGAGAACAC

RPLP0 fwd: GATGCCCAGGGAAGACAG rev: ACAATGAAGCATTTTGGATAATCA

RSP13 fwd: CGAAAGCACCTTGAGAGGAA rev: TTCCAATTAGGTGGGAGCAC

SCAP fwd: CTCAGGGGCAATCACAGC rev: TGAGCAAATGTTTGGCTGAC

dT TTTTTTTTTTTTTTTTTTTTTTTVN anchored oligo-dT mix for cDNA synthesis

N9 NNNNNNNNN random nonamers for cDNA synthesis 


\section{Methods}

\subsection{Animals and behaviour}

All investigations were performed in accordance with the German animal welfare law and the Lower Saxony State regulation for animal experiments. Animals were bred and kept in the animal facility of the Max Planck Institute of Experimental Medicine. Animals were kept in a 12h-light-dark-cycle and all handling and investigations were carried out in the light phase.

For this work C57BI6 animals were used as wildtype controls and homozygous CNP-Cre

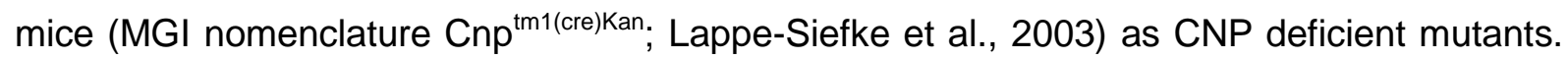
Animals were obtained from heterozygous breedings. Whenever possible, littermates of the same gender were housed in groups to reduce stress due to social isolation.

\section{Genotyping}

Tail biopsies were digested and gDNA harvested following manufacturer's instructions using the Genomic DNA-Isolation Kit for Tissue and Cells (NextTec). Eluted DNA was diluted 1:5 to exclude overload of the PCR and occurrence of unspecific bands. $0.5 \mu$ ldiluted DNA was used for genotyping.

CNP-PCR

$\begin{array}{ll}0.3 \mu \mathrm{l} & \text { Primer 4192 (10 pM) } \\ 0.3 \mu \mathrm{l} & \text { Primer 4193 (10 pM) } \\ 1 \mu \mathrm{l} & \text { Primer 2016 (10 pM) } \\ 1 \mu \mathrm{l} & \text { Primer 7315 (10 pM) } \\ 4 \mu \mathrm{l} & \text { 5x GoTaq Buffer } \\ 2 \mu \mathrm{l} & \text { dNTPs (10 mM) } \\ 0.1 \mu \mathrm{l} & \text { GoTaq } \\ 11.3 \mu \mathrm{l} & \text { dd }_{2} \mathrm{O}\end{array}$

Program:

$95^{\circ} \mathrm{C} \quad 180 \mathrm{~s}$ melting $56^{\circ} \mathrm{C} \quad 30 \mathrm{~s}$ annealing $72^{\circ} \mathrm{C} \quad 60 \mathrm{~s}$ elongation $95^{\circ} \mathrm{C} \quad 30 \mathrm{~s}$ melting back to step $2 \rightarrow 37$ cycles $56^{\circ} \mathrm{C} \quad 60 \mathrm{~s}$ final annealing $72^{\circ} \mathrm{C} \quad 600 \mathrm{~s}$ final elongation

For visualization GelRed was added in a $1: 20000$ ratio to each PCR reaction. PCR products were separated with $1.5 \%$ agarose gels in TBE buffer. The wildtype allele was presented by a $700 \mathrm{bp}$ band, whereas the CNP-Cre allele gave rise to a $400 \mathrm{bp}$ band. 


\section{Behaviour}

All sensory and motor behavioural tests were carried out blinded and with standardized methods. For sensory assessments animals were placed in the testing cages on the grid or plexiglass base of the dynamic plantar aesthesiometer (Ugo Basile, Borghi, Italy) and habituated to the setup for two hours. For mechanical sensitivity testing the plantar surface of the hindpaw was stimulated with a $0.5 \mathrm{~mm}$ Von-Frey-type filament. During testing phase the mechanical force increased by $1 \mathrm{~g} / \mathrm{s}$ and the stimulus intensity that induced paw withdrawal was recorded. If the paw was not lifted within $20 \mathrm{~s}$ the test was stopped to prevent tissue damage. Each animal was tested 5 times per hindpaw and the mean of all recordings per mouse was calculated. In order to assess the thermal sensitivity the plantar surface was irradiated with an infrared beam (40\% beam power). Paw withdrawal latencies were calculated from 5 independent trials per hindpaw per animal.

Additionally motor abilities of CNP and control animals were investigated. Therefore, the animal was placed on an $8 \mathrm{~mm}$ round bar with rough surface and the holding time (maximum $3 \mathrm{~min}$ ) was measured (Bar test). Results were displayed as sum of the 5 trials. Furthermore, in the grip strength test the animal had to grab a horizontal T-bar which was connected to a gauge. As the animal was pulled away the maximum force before the animal lost the grip was traced. The mean grip strength was calculated from five independent tests per mouse. Motor coordination was further investigated as the mouse had to pass a $2 \mathrm{~m}$ grid $(1 \mathrm{~cm}$ grid size) and all complete slips through the grid were counted (Grid slip test).

\section{Electrophysiological assessment}

Animals were anesthetized with Xylazine / Ketamine to have a time window of 20 min to perform all recordings under deep anesthesia. One pair of needle electrodes (Schuler Medizintechnik) was placed close to the sciatic notch (proximal) and one pair next to the tibial nerve in vicinity to the ankle (distal). Motor recording electrodes were positioned in the small foot muscle of the plantar surface. Compound muscle action potentials (CMAPs) were recorded after proximal and distal supramaximal stimulation of the sciatic and respective tibial nerve. The difference of responses, together with the distance of the stimulation electrodes allowed the calculation of the motor nerve conduction velocity (mNCV). For sensory recordings distal electrodes next to the tibial nerve were used for stimulation and the electrodes in proximity to the sciatic notch served as recording electrodes. The sensory nerve fibers were stimulated 10 times consecutively and the mean trace (sensory nerve potential) was calculated automatically. In combination with the distance of the electrodes the sensory nerve conduction velocity (sNCV) was calculated from the latency of the response. 


\subsection{Tissue preparation}

\section{$\underline{\text { Peripheral nerve preparation }}$}

Animals were sacrificed by cervical dislocation. Subsequently sciatic and saphenous nerves, as well as dorsal and ventral roots were carefully dissected. For immunohistological assessments nerves were immersion fixed for $24 \mathrm{~h}$ at $4^{\circ} \mathrm{C}$ in $4 \%$ PFA. Nerves for electron microscopic investigations were transferred to K\&S-fixative (4\% PFA and $2.5 \%$ glutardialdehyde, according to Schultz and Karlsson, 1965) which allows better structural preservation. To allow refined ultrastructural analyses saphenous nerves were cryofixed under high pressure (HPF). Therefore freshly dissected nerves were cut into suitable pieces and mounted in $0.2 \mathrm{~mm}$ carriers using $20 \%$ polyvinylpyrrolidone in PBS (PVP) as filler. Subsequently the samples were frozen under high pressure thus preventing tissue damage by ice crystal formation. HPF samples were stored in liquid nitrogen before freeze substitution. For RNA profiling or protein analyses the tissue was either snap frozen and stored at $-80^{\circ} \mathrm{C}$ or was immediately processed after dissection.

\section{$\underline{\text { Teased fiber preparation }}$}

Sciatic nerves of adult mice were freshly cut and transferred into cold PBS. Using fine forceps the endoneuria were carefully removed and nerves were split longitudinally into smaller proportions. Small nerve samples were transferred to a droplet of cold PBS on a superfrost slide. Thereafter, fibers were carefully separated to achieve areas where axons are completely separated from each other. Reaching a good separation of fibers the samples were dried. Teased fibers were stored at $-20^{\circ} \mathrm{C}$ if not processed immediately.

\subsection{Electron microscopy}

K\&S-fixed nerves were postfixed in $2 \%$ osmium tetraoxide and subsequently dehydrated and embedded in epoxy resin (protocol page 35). High pressure frozen material was processed using $0.1 \%$ tannic acid as mordant and $2 \%$ osmium tetraoxide for postfixation. Subsequent steps of the freeze substitution (protocol page 35) from warm up and dehydration over epon infiltration to final polymerization were performed automated using the AFS II (Leica). Epon embedded nerves were cut in longitudinal or transverse direction as $0.5 \mu \mathrm{m}$ semithin or $50 \mathrm{~nm}$ ultrathin sections (Ultracut $S$ ultramicrotome, Leica). Semithin sections were stained with methylene blue / Azure II blue (according to Richardson, 1960) for 1 min to visualize lipid rich tissue (myelin). Ultrathin sections were contrasted with $4 \%$ uranyl acetate for 30 min 
and lead citrate for $7 \mathrm{~min}$ (according to Reynolds, 1963). The contrasting of ultrathin sections of HPF samples was adapted to 15 min uranyl acetate and 3 min lead citrate incubation. Electron micrographs were taken with the EM900 (Zeiss; 3.500x - 30.000x magnification) or EM912AB (Zeiss; high magnification) electron microscopes which are equipped with wideangle dual speed 2K-CCD-Cameras (TRS, Moorenweis) using the ImageSP software (TRS, SysProg, ImageSP vers. 1.1.4.62). Image analysis was carried out with ImageJ vers1.42q.

\subsection{Histology}

After peripheral nerves had been immersion fixed for $24 \mathrm{~h}$ in 4\% PFA they were washed with PBS and embedded in 2\% low melting agarose (BactoAgar, BD) to allow for easy arrangement of nerves. The tissue was dehydrated, paraffin wax infiltrated (protocol page 36) and positioned in wax blocks. $5 \mu \mathrm{m}$ sections were assembled and mounted on slides and dried over night at $37^{\circ} \mathrm{C}$. For stainings sections were rehydrated (protocol page 36).

\section{$\underline{\mathrm{DAB}}$}

Within the rehydrated sections the endogenous peroxidase was inactivated by incubation with 3\% hydrogen peroxide for 5 min followed by several washing steps with Tris buffered milk. To reduce unspecific staining, sections were incubated for $20 \mathrm{~min}$ in blocking solution (containing inactivated goat serum and bovine serum albumin) followed by overnight incubation with the primary antibody (for dilution see page 24) in Antibody-Diluent (DAKO) at $4^{\circ} \mathrm{C}$. Subsequently, sections were washed with Tris buffered milk and incubated for $30 \mathrm{~min}$ at RT with the specific biotinylated secondary antibody in Antibody-Diluent (DAKO). After washing the sections with Tris buffered milk the streptavidin-coupled HRP-conjugated bridging antibody was applied following manufacturers instructions (Vectastain Elite ABC kit; Vector). Repeated washing steps with Tris-buffer were followed by the enzyme based detection of the antigen with the help of the diaminobenzidine (DAB) precipitation reaction (DAB Substrate Kit, Zytomed). Afterwards, the sections were counterstained with hematoxylin and dehydrated (protocol page 36 ) before mounting.

\section{Fluorescent labeling}

For immunofluorescence labeling rehydrated sections were blocked for 20 min with goat serum and bovine serum albumin followed by overnight incubation (at $4^{\circ} \mathrm{C}$ ) with primary antibodies in blocking solution (for dilutions see page 24). Following subsequent washing steps with Tris buffered milk, sections were incubated for 60 min with the corresponding fluorescently labeled secondary antibodies. DAPI was commonly added to the secondary 
antibody dilutions to gian a nuclear counterstain. Finally, sections were washed and mounted with Aquapolymount.

\section{Teased fiber staining}

Teased fibers were postfixed on slide for $5 \mathrm{~min}$ in freshly prepared $4 \%$ PFA and permeabilized with icecold methanol for additional $5 \mathrm{~min}$. After three subsequent washes with phosphate buffered saline (PBS) for 5 minutes, the fibers were blocked for $2 \mathrm{~h}$ with 10\% horse serum and $0.1 \%$ Tween20 in PBS at room temperature. Hereafter, the tissue was incubated over night at $4^{\circ} \mathrm{C}$ with the primary antibodies (dilutions see page 24). The slides were washed three times with PBS. Appropriate secondary antibodies (page 24) were applied to the fibers for 1 hour at room temperature. After final washing with PBS fibers were mounted with Aquapolymount.

\section{Light microscopy}

Light microscopic images of semithin sections or DAB stainings were taken with the Zeiss Image Z1 microscope. For overview images the 20x (Plan-Neofluar 20x 0.50 Air; Zeiss) and for tile images the 100x (Plan-Neofluar 100x 1.30 Oil, Zeiss) objectives were used. Images were taken with the help of the ZEN2011 (Zeiss) software. Fluorescent images were captured with the Axiophot Observer $Z$ (inverted, Zeiss). Overview images were taken with the 20x objective, whereas high magnification images were taken using the $63 \mathrm{x}$ (CApochromat, Zeiss) objective. For image acquisition ZEN2012 (Zeiss) software and for image analysis ImageJ vers1.42q software was used.

\subsection{RNA isolation and analysis}

\section{$\underline{\text { RNA extraction }}$}

Snap frozen or freshly prepared dorsal and ventral roots were homogenized in Trizol (Invitrogen) by shaking twice for $10 \mathrm{~s}$ at $5500 \mathrm{rpm}$ using the Precellyse24 (Bertin Technologies). Total RNA was extracted following manufacturer's instructions for "RNA extraction from lipid rich tissue" with the help of RNeasy Mini- or MicroRNA Kits (Qiagen). RNA concentrations and qualities were analyzed with Agilent RNA 6000 Nano Kits (Agilent) or the NanoDrop200 Spectraphotometer (Thermo Scientific). 


\section{$\underline{\text { cDNA synthesis }}$}

For quantitative mRNA comparisons equal amounts of total RNA (per experiment $200 \mathrm{ng}$ $1 \mu \mathrm{g}$ ) were reversely transcribed to cDNA providing excess of random nonamers (N9) and oligodesoxythymidine primers (dT) as well as dNTPs using the SuperScript III reverse transcriptase (Invitrogen).

First strand cDNA synthesis

$8 \mu \mathrm{l} \quad$ RNA ( 500 ng)

$2 \mu \mathrm{l} \quad$ Oligo dT mix primer $(0.6 \mu \mathrm{M})$

$2 \mu \mathrm{l} \quad \mathrm{N} 9$ random nonamer primer $(0.12 \mathrm{mM})$

Incubated for 2 min at $70^{\circ} \mathrm{C}$ to denature all double stranded RNAs and primers. Briefly put on ice before proceeding.

$4 \mu \mathrm{l} \quad 5 \times 1^{\text {st }}$ strand buffer

$2 \mu \mathrm{l} \quad 100 \mathrm{mM} \mathrm{DTT}$

$2 \mu \mathrm{l} \quad 10 \mathrm{mM}$ dNTPs

$1 \mu \mathrm{l} \quad$ SuperScript III reverse transcriptase $(200 \mathrm{U} / \mu \mathrm{l})$

Mastermix 2 was added to each reaction. The final $20 \mu \mathrm{l}$ reaction was incubated for $10 \mathrm{~min}$ at $25^{\circ} \mathrm{C}$ followed by $45 \mathrm{~min}$ incubation at $50^{\circ} \mathrm{C}$ and final incubation at $55^{\circ} \mathrm{C}$ for another $45 \mathrm{~min}$. Synthesized cDNAs were diluted to $1 \mathrm{ng} / \mu \mathrm{l}$.

\section{$\underline{\text { Quantitative real time polymerase chain reaction ( } \mathrm{RRT}-\mathrm{PCR})}$}

Quantitative real time PCRs were carried out using the SybrGreen system (Applied Biosystems) and 1-2 ng of cDNA per reaction.

Pipette scheme per reaction (4 technical replicates were used)

$2 \mu \mathrm{l} \quad \mathrm{cDNA}$

$5 \mu \mathrm{l} \quad$ Power SybrGreen master mix

$0.2 \mu \mathrm{l} \quad$ forward primer $(100 \mu \mathrm{M})$

$0.2 \mu \mathrm{l} \quad$ reverse primer $(100 \mu \mathrm{M})$

$2.6 \mu \mathrm{l} \quad \mathrm{ddH}_{2} \mathrm{O}$

Program:

$50^{\circ} \mathrm{C} \quad 120 \mathrm{~s}$ warm up

$95^{\circ} \mathrm{C} \quad 600 \mathrm{~s}$ melting

$60^{\circ} \mathrm{C} \quad 20 \mathrm{~s}$ annealing

$72^{\circ} \mathrm{C} \quad 30 \mathrm{~s} \quad$ elongation and detection

$95^{\circ} \mathrm{C} \quad 10 \mathrm{~s} \quad$ melting $\rightarrow$ back to step $3 \rightarrow 45$ cycles 
Raw data were collected with the Light cycler 480 (Roche, 384 well plates) or the 7500 Fast real-time PCR system (Applied Biosystems, 96 well plated), both equipped with according softwares (LightCycler®480 software 1.5.0SP4 / 7500FastSystem SDS software). Relative quantities $(R U)$ were calculated from the four technical replicates using the internal software. Data analysis was carried out using Microsoft Excel 2007. To standardize for differences in the cDNA input, all tested genes were normalized to the following standard genes: ribosomal protein large P0 (RPLP0), ribosomal protein S13 (RSP13), Cyclophilin (CycPh) and betaActin (bAct). The average of all biological replicates was calculated and all values were related to wildtype dorsal root levels that were set to 1 .

\subsection{Proteinbiochemical analysis}

\section{$\underline{\text { Protein extraction }}$}

Freshly dissected or snap frozen material was homogenized in sucrose buffer, which additionally contained protease inhibitors as well as phosphatase inhibitors if neccessary. Protein concentrations of the homogenates were measured using the BioRad DC ${ }^{\mathrm{TM}}$ Protein Assay system (BioRad) according to producer's protocol. First, bovine serum albumin in concentrations from $0.2-3 \mu \mathrm{g} / \mu \mathrm{l}$ was measured to calculate a standard curve. All samples were quantified relative to the standards. To ensure loading of equal amounts samples were adjusted to the same concentration.

\section{Myelin protein purification}

Most protein biochemical analyses were carried out on purified myelin. Therefore preferably freshly dissected peripheral nerve tissue was homogenizes (Precellyse24: 2x 10s at $5500 \mathrm{rpm}$ ) in $1 \mathrm{ml}$ of $0.29 \mathrm{M}$ sucrose containing protease inhibitors. Centrifugation tubes were prepared with $1 \mathrm{ml}$ of $0.85 \mathrm{M}$ sucrose solution. The $1 \mathrm{ml}$ homogenates were carefully layered over the $0.85 \mathrm{M}$ sucrose without mixing the solutions. After centrifugation at $35000 \mathrm{rpm}$ (using the TLS55 rotor, $\sim 75000 \mathrm{~g}$ ) for $30 \mathrm{~min}$, the interphases were transferred to new centrifugation tubes and clean water was added in excess to wash out the sucrose. These solutions were centrifuged for another $20 \mathrm{~min}$ at $35000 \mathrm{rpm}$ (TLS55 rotor, 75.000 g) to receive a protein pellet. Supernatants were carefully removed. Pellets were resuspended in clean water and incubated for $15 \mathrm{~min}$ on ice to solve cytoplamic proteins. After final low speed centrifugation at $16000 \mathrm{rpm}$ for $15 \mathrm{~min}$ (TLS55 rotor, 12000 g) the supernatants were removed carefully and myelin protein pellets were resolved in a small volume $(20-50 \mu \mathrm{l})$ of $1 \mathrm{x}$ Tris buffered saline. Protein concentrations were measured as described above. 


\section{Protein separation with SDS-PAGE}

Freshly prepared SDS-polyacrylamide gels $(10-16 \%)$ were loaded with $1-20 \mu \mathrm{g}$ of total homogenate or purified myelin (in protein loading dye with 5\% B-mercapthoethanol). Only for detection of the myelin associated glycoprotein (MAG) non-reducing conditions were chosen. After focusing the proteins in the stacking gel at low voltage, protein separation was carried out at $\sim 80 \mathrm{~V}$ using the BioRad Protein Electrophoresis device. Single proteins herein separated according to their charge, which corresponds to their size.

\section{$\underline{\text { Western Blotting }}$}

Proteins were transferred to methanol activated polyvinylidene difluoride (PVDF) membranes by wet chamber western blotting (BioRad). Therefore sandwiches of filter, filterpapers, gel and membrane were inserted into the wet chamber, filled with cold transfer buffer. According to the size of the protein of interest, gels were blotted for $1.5-2 \mathrm{~h}$ at $80 \mathrm{~V}$ and $4^{\circ} \mathrm{C}$. Afterwards, gels were incubated in Brilliant blue G250 coomassie (BioRad) to control for the transfer of proteins and equal loading. Membranes were subsequently blocked for $1 \mathrm{~h}$ in either 5\% milk in TBST or in 5\% BSA in TBST for most phosphorylated proteins. In the following primary antibody incubations were carried out over night at $4^{\circ} \mathrm{C}$ while rotating the membranes (For antibody dilutions see page 24). Membranes were washed four times for $15 \mathrm{~min}$ in TBST to get rid of unbound primary antibodies. Afterwards appropriate secondary antibodies were applied for $45 \mathrm{~min}$ at room temperature. Following several washes with TBST, proteins were detected using the Western Lightning Plus-ECL-Kit (Perkin Elmer Life Sciences). To control for equal loading membranes were incubated with actin or GAPDH as internal standards. Quantifications of immunoblots were carried out using the "Gele" analysis plugin of ImageJ. All values were normalized to the standard proteins on the same gel. Quantities were displayed as relative values related to wildtype dorsal roots that were set to 1.

\section{Silver impregnation of SDS-gels}

Silver impregnations were performed to unspecifically visualize all proteins within peripheral nerve lysates or purified myelin samples. Therefore, $0.5 \mu \mathrm{g}$ proteins were loaded on freshly prepared SDS-gels. SDS-PAGE was performed as described above. After separation of proteins the gels were silver stained according to a protocol modified from Blum (1987) and Mortz (2001). First, gels were fixed with $40 \%$ ethanol, $10 \%$ acetic acid for $1 \mathrm{~h}$ and washed twice with $30 \%$ ethanol and once with $\mathrm{ddH}_{2} \mathrm{O}$ for 20 min each. The fixed gels were then 
incubated with sodium thiosulfate $(0.8 \mathrm{mM})$ for 1 min followed by short washes in $\mathrm{dd}_{2} \mathrm{O}$ for $1 \mathrm{~min}$ in total. The gels were further impregnated with $0.2 \%$ silver nitrate (containing $0.05 \%$ formaldehyde solution (37\%)) for $20 \mathrm{~min}$ and rinsed with $\mathrm{dd}_{2} \mathrm{O}$ for $1 \mathrm{~min}$. For subsequent chemical development the prepared gels were incubated with $3 \%$ sodium carbonate (containing $0.05 \%$ formaldehyde solution (37\%)) until the staining was sufficient. Replacing the developing solution by $5 \%$ acetic acid stopped the reaction. The stainings were fixed by 10 min incubation with $5 \%$ acetic acid followed by $3 \times 10$ min washes with water to flush all remaining chemicals.

\section{Proteome analysis}

Myelin was purified from dorsal and ventral roots of adult animals ( $n=3$ per group). All samples were analyzed with silver staining to ensure equal qualities of the myelin preparation. The purified myelin was used for gel-free proteome analysis which was carried out by Dr. Stefan Tenzer at the Institute of Immunology, University of Mainz. Each sample was analyzed in 4 technical replicates. Thus, purified proteins were digested with trypsin to receive shorter peptides that have subsequently been investigated using mass spectrometry (LC-MS). The peptide sequences were aligned with the UniProtKB/SwissProt Protein Knowledgebase (mouse protein data set) for protein identification (detailed description in Patzig et al., 2011). The quantitative dorsal root myelin protein data were compared to data obtained for ventral root myelin to unravel differences in protein expression.

\subsection{Data analysis}

All technical replicates were combined to one mean data point for each sample. Biological replicates were single data points (n-numbers). For each group (time points or genotypes) the mean, standard deviation (SD) and the standard error of mean (SEM) was calculated. When the statistical comparison only included two groups (e.g. wildtype versus CNP deficient mice) the student's t-test was performed using Excel 2007. Calculated p-values above 0.05 were assigned as not significant (ns) in the diagrams. When the statistical test yielded $p$ values smaller than 0.05 the comparison was considered as significantly different. P-values smaller than 0.05 are displayed with $\left({ }^{*}\right),>0.01$ with $\left(^{* *}\right)$ and $p$-values below 0.001 with $\left(^{* * *}\right)$. When multiple comparisons had to be performed 2-way-Anova tests with Bonferroni post hoc test and frequency distributions were performed using GraphPad Prism vers. 5.04. 


\subsection{Embedding protocols}

\section{$\underline{\text { Conventional embedding of tissue in Epoxy resin protocol }}$}

0.1 M Phosphate buffer (PB) $\quad 3 \times 10 \mathrm{~min} \quad 4^{\circ} \mathrm{C}$

$2 \%$ Osmium tetraoxide in $0.1 \mathrm{M} \mathrm{PB} 4 \mathrm{~h} \quad 4^{\circ} \mathrm{C}$

$0.1 \mathrm{M}$ Phosphate buffer $\quad 3 \times 10 \mathrm{~min} \quad 4^{\circ} \mathrm{C}$

$30 \%$ Ethanol $20 \mathrm{~min} \quad 4^{\circ} \mathrm{C}$

$50 \%$ Ethanol $20 \mathrm{~min} \quad 4^{\circ} \mathrm{C}$

$70 \%$ Ethanol $\quad 20 \mathrm{~min} \quad 4^{\circ} \mathrm{C}$

$90 \%$ Ethanol $20 \mathrm{~min} \quad 4^{\circ} \mathrm{C}$

$100 \%$ Ethanol $\quad 3 \times 10 \mathrm{~min} \quad 4^{\circ} \mathrm{C}$

2-Propanol $\quad 10 \mathrm{~min} \quad 4^{\circ} \mathrm{C}$

Propylene oxide $\quad 3 \times 10 \mathrm{~min} \quad \mathrm{RT}$

$2: 1$ Propylene oxide : Epon $2 \mathrm{~h} \quad \mathrm{RT}$

$1: 1$ Propylene oxide : Epon $2 \mathrm{~h} \quad \mathrm{RT}$

$1: 2$ Propylene oxide : Epon $\quad 4 \mathrm{~h} \quad \mathrm{RT}$

pure Epon $4 \mathrm{~h} \quad \mathrm{RT}$

Transfer tissue from bucket to embedding molds and polymerize for $24 \mathrm{~h}$ at $60^{\circ} \mathrm{C}$

\section{Automated freeze substitution protocol}

$\begin{array}{lll}0.1 \% \text { Tannic acid in acetone } & 100 \mathrm{~h} & -90^{\circ} \mathrm{C} \\ \text { Icecold acetone } & 4 \times 30 \mathrm{~min} & -90^{\circ} \mathrm{C} \\ 2 \% \text { Osmium tetraoxide in acetone } & 7 \mathrm{~h} & -90^{\circ} \mathrm{C} \\ & 14 \mathrm{~h} & \text { warm up to }-20^{\circ} \mathrm{C} \text { by } 5^{\circ} \mathrm{C} / \mathrm{h} \\ & 16 \mathrm{~h} & -20^{\circ} \mathrm{C} \\ & 2.4 \mathrm{~h} & \text { warm up to } 4^{\circ} \mathrm{C} \text { by } 10^{\circ} \mathrm{C} / \mathrm{h} \\ & 1 \mathrm{~h} & 4^{\circ} \mathrm{C} \\ \text { cold acetone } & 3 \times 20 \text { min } & 4^{\circ} \mathrm{C} \\ 1: 1 \text { Epon : acetone } & 3 \mathrm{~h} & \mathrm{RT} \\ 90 \% \text { Epon in acetone } & \text { overnight } & \text { RT } \\ \text { Pure Epon (freshly made) } & 6 \mathrm{~h} & \text { RT }\end{array}$

Remove nerve tissue from sample carrier and place in suitable embedding mold. Polymerization for $24 \mathrm{~h}$ at $60^{\circ} \mathrm{C}$. 
Paraffin wax embedding protocol

$\begin{array}{lll}50 \% \text { Ethanol } & \text { once } & 1 \mathrm{~h} \\ 70 \% \text { Ethanol } & \text { twice } & 2 \mathrm{~h} \\ 96 \% \text { Ethanol } & \text { twice } & 1 \mathrm{~h} \\ 100 \% \text { Ethanol } & \text { twice } & 1 \mathrm{~h} \\ 2-\text { Propanol } & \text { once } & 1 \mathrm{~h} \\ \text { Xylol } & \text { twice } & 2 \mathrm{~h} \\ \text { Paraffin wax }\left(60^{\circ} \mathrm{C}\right) & \text { twice } & 2 \mathrm{~h}\end{array}$

$\underline{\text { Rehydration protocol for wax sections }}$

$\begin{array}{lll}\text { Warm up } & 10-20 \mathrm{~min} & 60^{\circ} \mathrm{C} \\ \text { Xylene } & \text { twice } 10 \mathrm{~min} & \mathrm{RT} \\ 1: 1 \text { Xylene : 2-Propanol } & 10 \mathrm{~min} & \mathrm{RT} \\ 100 \% \text { Ethanol } & 5 \mathrm{~min} & \mathrm{RT} \\ 90 \% \text { Ethanol } & 5 \mathrm{~min} & \mathrm{RT} \\ 70 \% \text { Ethanol } & 5 \mathrm{~min} & \mathrm{RT} \\ 50 \% \text { Ethanol } & 5 \mathrm{~min} & \mathrm{RT} \\ \mathrm{ddH}_{2} \mathrm{O} & 5 \mathrm{~min} & \mathrm{RT} \\ \text { Citrate buffer } & 5 \mathrm{~min} & \mathrm{RT} \\ \text { Citrate buffer } & 10-15 \mathrm{~min} & \text { boiling in microwave } \\ & 20 \mathrm{~min} & \text { cooling down to RT } \\ \text { Tris buffered milk } & 5 \mathrm{~min} & \mathrm{RT}\end{array}$

$\underline{\text { Counterstaining with hematoxylin (for wax sections) }}$

$\begin{array}{ll}\text { Mayer's hematoxylin solution } & 30 \mathrm{~s} \\ \mathrm{ddH}_{2} \mathrm{O} & \text { shortly } \\ \mathrm{HCl}-\text { Alcohol } & \text { few seconds } \\ \mathrm{ddH}_{2} \mathrm{O} & \text { shortly } \\ \mathrm{Scotts} \text { solution } & 5 \mathrm{~min} \\ \mathrm{ddH}_{2} \mathrm{O} & \text { shortly } \\ 50 \% \text { Ethanol } & 5 \mathrm{~min} \\ 70 \% \text { Ethanol } & 5 \mathrm{~min} \\ 90 \% \text { Ethanol } & 5 \mathrm{~min} \\ 100 \% \text { Ethanol } & 5 \mathrm{~min} \\ 1: 1 \text { Xylene : 2-Propanol } & 10 \mathrm{~min} \\ \text { Xylene } & \text { twice } 10 \mathrm{~min}\end{array}$




\section{Results}

\subsection{Schwann cells form distinct myelin sheaths}

Peripheral nerves comprise all kinds of nerve fibers, ranging from very small unmyelinated predominantly sensory fibers to large strongly myelinated motor fibers. Associated glial cells are grouped into myelinating and nonmyelinating Schwann cells (SC) (Ochoa and Mair, 1969; reviewed in Jessen and Mirsky, 2005). Within the group of myelinating SC differences in the expression of trophic factors and extracellular matrix components have been described (Höke et al., 2006; Madison et al., 2009). To date it has not been investigated if SC that wrap small compared to large caliber axons also differ with regard to their myelin sheath. Thus, the focus of this work is the comparison of the myelin sheaths of motor and sensory SC and the investigation of the role of specific myelin proteins for discrete fibers types. I took advantage of the well described separation of small and large fibers in dorsal (small / sensory) and ventral (large / motor) roots. To demonstrate the differences, semithin sections of wildtype dorsal and ventral roots were prepared as shown in Fig 1. It is easy to identify the dorsal root (left) that contains all kinds of sensory fibers from small unmyelinated C-fibers in Remak bundles, up to larger, slow and also fast conducting A-alpha, beta and delta afferent fibers. In contrast, ventral roots comprise large A-alpha type motor axons which are well myelinated. Additionally they contain a minor proportion of small A-beta and A-gamma motor neurons that innervate muscle spindles (FitzGerald et al., 2011).

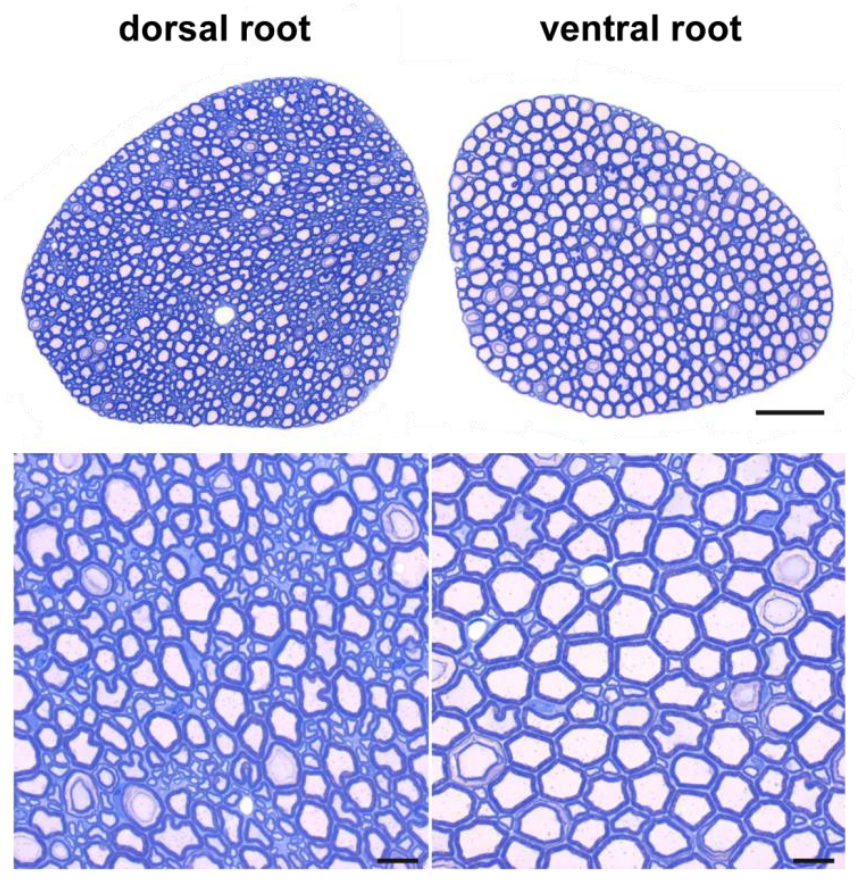

Fig 1: Dorsal and ventral roots contain different fiber types

Semithin cross section of a wildtype dorsal and ventral root (height L4) stained with methylene blue / azure II blue, which displays myelin (blue) and respectively the (unstained) axons. Bottom images more clearly depict differences in fiber sizes of dorsal (left) and ventral (right) roots. (Scale $50 \mu \mathrm{m}$ in overview, bottom scale $10 \mu \mathrm{m}$ ). 


\subsubsection{Distinct myelin composition in dorsal and ventral roots}

Motor and sensory SC exhibit differences regarding extracellular matrix components and growth factors during peripheral nerve regeneration. We hypothesized that motor and sensory SC also differ with regard to their myelin sheaths. Initial comparison of myelin purified from uninjured wildtype dorsal and ventral roots of adult animals showed that the myelin of dorsal and ventral roots indeed display a different composition when proteins were visualized by silver impregnation after SDS-polyacrylamide gelelectrophoresis. The majority of proteins showed stronger band intensities in myelin of the dorsal roots (left arrowheads in Fig 2A). For example CNP, which runs at $46 \mathrm{kDa}$ and $48 \mathrm{kDa}$ showed a stronger signal in dorsal root myelin (highlighted with "CNP"). Additionally, some proteins were enriched in ventral root myelin (right arrowheads in Fig 2A). Mass spectrometry (MS) based quantitative proteome analyses furthermore displayed differentially regulated proteins. Here, the relative abundance of proteins purified from dorsal root myelin was compared to their abundance in ventral root myelin and all differentially expressed proteins were categorized regarding their described functions. Remarkably, these differentially expressed proteins not only comprise classical compact and noncompact myelin proteins, but also proteins linked to the cytoskeleton, cytoskeleton dynamics, enzymes and proteins related to energy metabolism of the cells (Fig 2B). Interestingly, in this MS based comparison the majority of identified proteins were significantly increased in ventral roots. Among those ventrally enriched proteins most seem to be of generally low abundance and may thus not be represented by the protein bands in the silver gels which show only relatively abundant proteins. However, more subtle differences as observed in this first proteome comparison may exist. These observations imply a general diversity within the myelinating SC population and the differences in protein composition likely display individual adaptations of SC to their engulfed axon. 


\section{A}

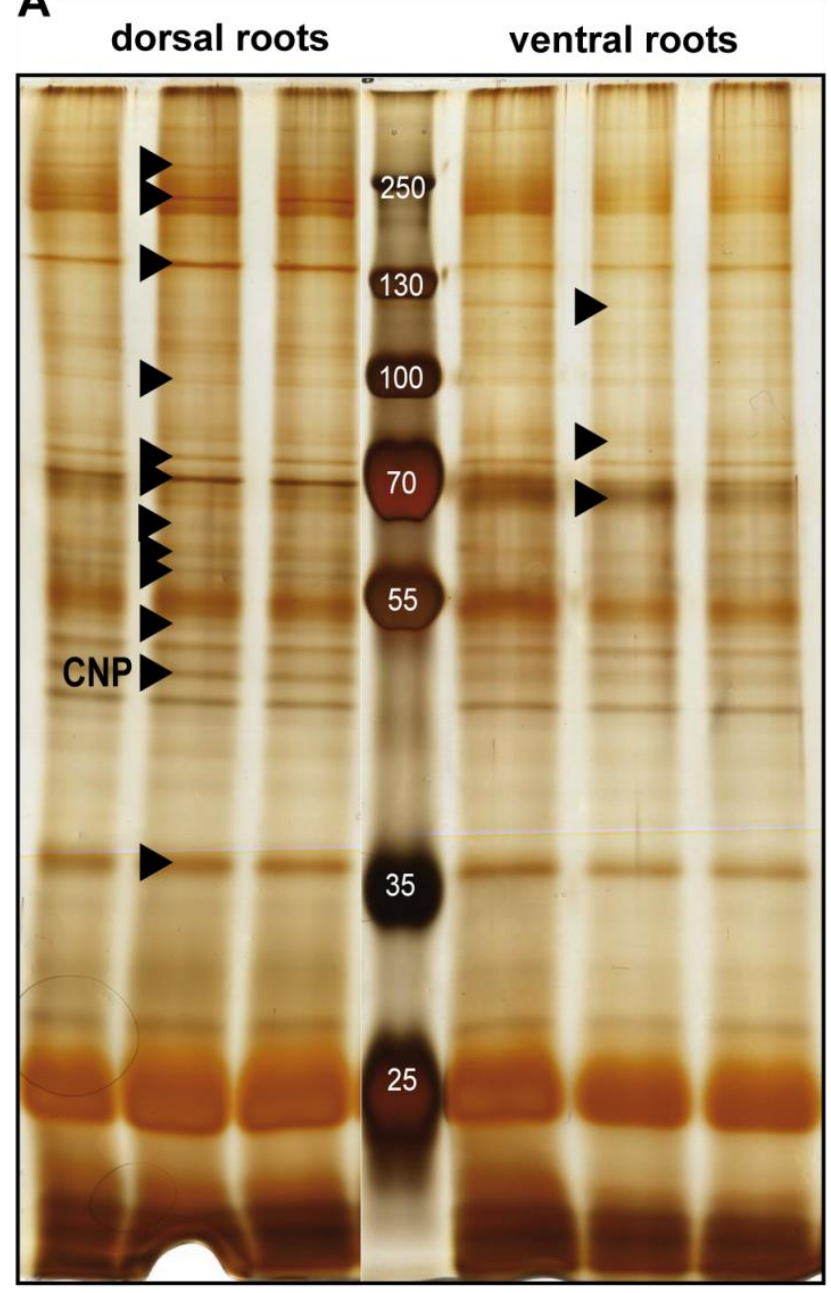

\section{B}

\section{comparative proteome analysis of dorsal and ventral root myelin}

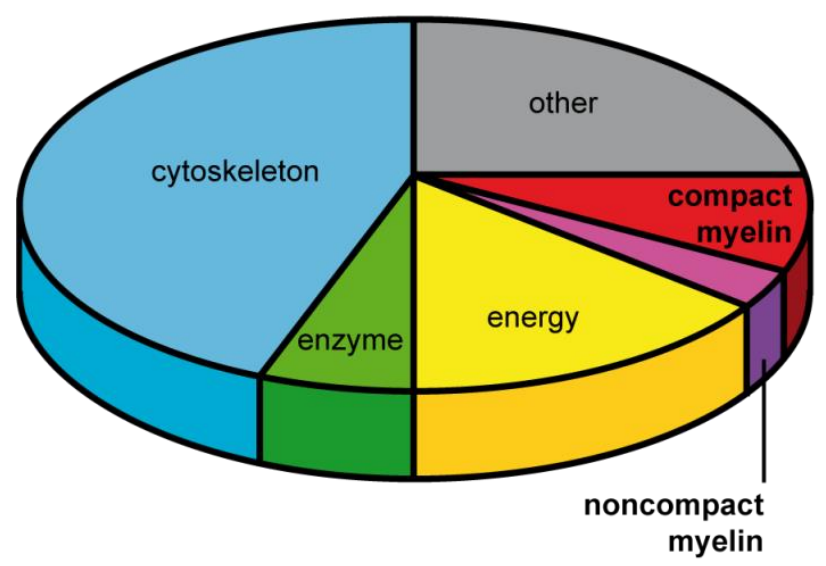

Fig 2: Purified myelin of dorsal and ventral roots displays different protein compositions

A: Silver impregnation of a SDS-gel loaded with purified myelin from dorsal and ventral roots. Arrowheads highlight proteins that are more abundant in dorsal roots (left) or ventral roots (right) when comparing band intensities. This demonstrates a differential protein composition in motor and sensory SC ( $\mathrm{n}=3$ per group).

B: Mass spectrometry based comparisons of dorsal and ventral root myelin proteomes. All proteins that are differentially expressed are included in the pie chart. Categorization of these proteins reveals that not only classical compact and noncompact myelin proteins but also proteins related to cytoskeleton, enzymes and energy functions display differences in their abundance in motor and sensory SC. ( $n=3$ per group). 


\subsubsection{Different abundance of myelin proteins in dorsal and ventral roots}

The expression levels of classical myelin proteins were further investigated on immunoblot level and remarkable differences were detected between myelin of dorsal and ventral roots. The noncompact myelin protein 2',3'-cyclic nucleotide 3'-phosphodiesterase (CNP) displayed a robust difference in protein abundance with increased expression levels in dorsal roots (Fig 3A). In addition, Sirtuin2, which is a NAD ${ }^{+}$dependent deacetylase (Werner et al., 2007), as well as Connexin29 (localized to all cytoplasmic regions of Schwann cells, Altevogt et al., 2002) and Septin2, which is part of the Septin filament skeleton underneath the myelin sheath (Buser et al., 2009) appear to be elevated in dorsal compared to ventral roots. Differences in protein abundance were not only observed for noncompact myelin proteins but also among the tested compact myelin proteins. The proteolipid protein (PLP and its shorter isoform DM20), which is one of the most abundant myelin proteins (Garbern et al., 1997), was found to be even more enriched in Schwann cells surrounding small sensory fibers (dorsal root) compared to Schwann cells engulfing large motor fibers in the ventral root (Fig 3B). Of the myelin proteins tested, only the peripheral myelin protein 2 (MyP2), which is barely detectable in dorsal roots, showed elevated expression levels in ventral roots of adult wildtype animals. This is in line with findings by other groups (Kadlubowski et al., 1984). However, other investigated myelin proteins like Caspr, MAG, PMP22 and MBP did not show obvious differences in their expression levels comparing motor and sensory nerves. To clarify myelin protein abundance differences, band intensities were quantified. Proteins of interest were normalized to their respective loading control (Actin or GAPDH). Data are presented relative to the protein expression level in ventral roots, which was set to 1 (Fig 3C). Among the analyzed proteins CNP showed the most robust difference in expression with 2.7 times higher protein levels in dorsal compared to ventral root samples. These data confirmed the differences that were observed in the proteome analysis. Moreover they prompted at CNP as a candidate protein to study the differential impact of myelin proteins for the integrity of distinct peripheral nerve fiber types. 
A

noncompact

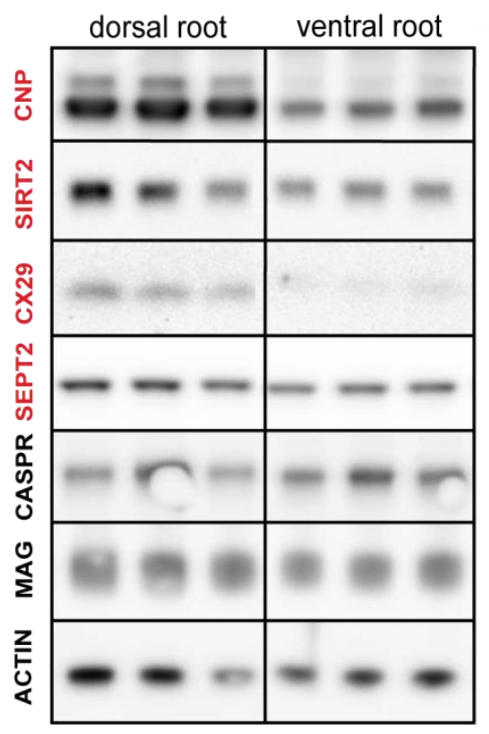

B

compact

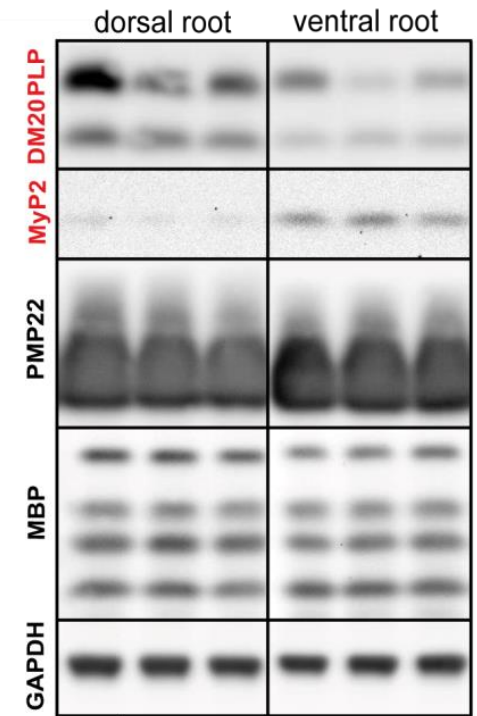

C
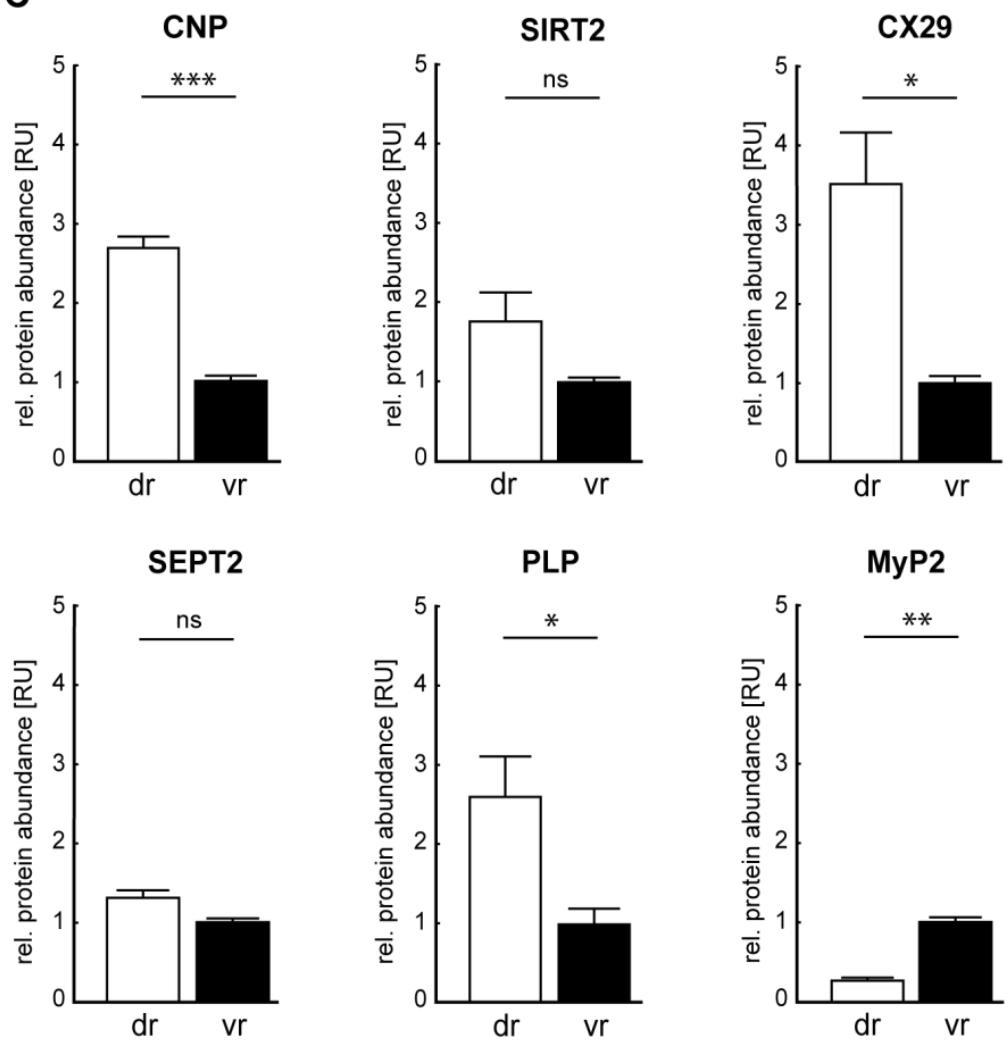

Fig 3: Distinct myelin protein abundances in dorsal and ventral roots

A: Immunoblots of classical noncompact myelin proteins in dorsal and ventral root lysates from 6 months old wildtype animals. CNP shows a remarkable enrichment in dorsal compared to ventral roots. Additionally, Sirtuin2 (Sirt2), Connexin 29 (CX29) as well as Septin2 (SEPT2) are elevated in dorsal roots. ( $\mathrm{n}=3$ per group).

B: Also the classical compact myelin proteins PLP/DM20 and MyP2 display different expression levels in dorsal compared to ventral roots, while others (PMP22 and MPB) are of equal abundance. ( $n=3$ per group).

C: Relative quantification of compact and noncompact myelin protein expression levels in dorsal and ventral root samples from 6 months old wildtypes. CNP is 2.7 times stronger expressed in dorsal roots compared to ventral roots and displays highly significant differences. Also MyP2 which is barely detectable in dorsal roots shows robust difference in its expression with elevated levels in the ventral root. Bar diagrams are only displayed for myelin proteins that appeared differentially regulated on the immunoblots in $A$ and $B$ (highlighted in red). ( $n=3$ per group; Mean + SEM). 


\subsection{CNP is elevated in myelinating Schwann cells of dorsal roots}

\subsubsection{Histological confirmation of increased CNP levels in dorsal roots}

CNP abundance differences have not only been detected on protein level in immunoblots but were further observed when peripheral nerve tissue was stained with an anti-CNP-antibody. Here, dorsal roots displayed numerous CNP positively labeled SC (green), which were only rarely observable in their ventral root counterparts (Fig 4). To unravel the specificity of the CNP signal for SC of different fiber populations, the CNP staining intensities were correlated to the size of the associated fibers. Therefore axons were visualized with the neuron specific beta-Tubulin III marker (Tuj1, grey), which allows to discriminate between large and small fibers. The vast majority of CNP positive staining was associated with small caliber axons. This explains, why the overall staining intensity was much stronger in dorsal compared to ventral roots, because the proportion of small fibers is much higher in dorsal compared to ventral roots. As it is easy to discriminate large and small fibers in ventral roots, a high magnification image of a ventral root shall illustrate the clear association of the SC specific CNP-signals (green) with small axons (Tuj1, staining in grey). These observations were in line with the higher CNP protein levels in dorsal roots seen on the immunoblots.

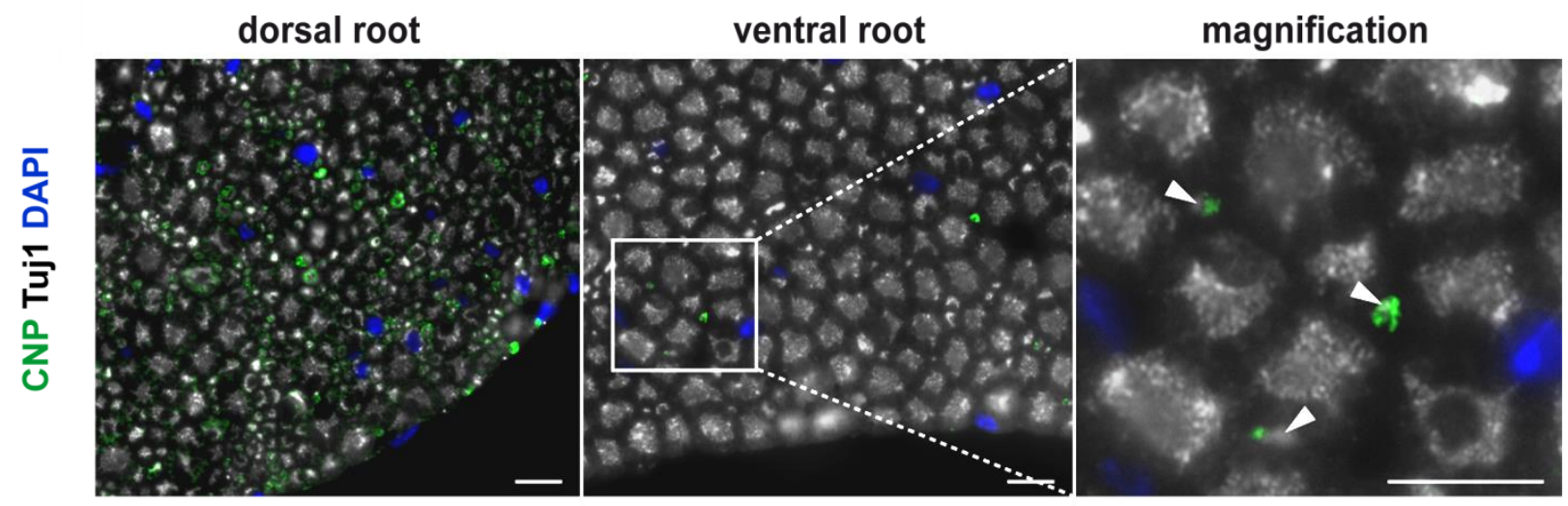

Fig 4: Staining intensity for CNP is higher in dorsal roots

Wax sections of dorsal and ventral roots of 6 months old wildtype animals stained for CNP (green) as well as Tuj1 (grey, to display corresponding axons) and DAPI (nuclear stain). Dorsal roots show more CNP positive areas than respective ventral roots. CNP stained Schwann cells are mostly associated with small caliber axons (magnification). (Scale bars depict $10 \mu \mathrm{m}$ ). 


\subsubsection{CNP transcript levels are enhanced in dorsal root Schwann cells}

Furthermore, quantitative real time PCR (qRT-PCR) analyses comparing CNP specific mRNA abundances in total RNAs from dorsal and ventral root samples revealed elevated CNP transcript levels in dorsal root Schwann cells (Fig 5). Relative to the ventral root level, which was set to one, dorsal roots exhibited 55\% more CNP mRNA. Thus, CNP seems to be differentially regulated on transcriptional and translational level, with more RNA and protein expressed in dorsal compared to ventral root Schwann cells. All these observations raised the question whether different CNP expression levels correlate with a differential impact of CNP function on small versus large peripheral nerve fibers.

A

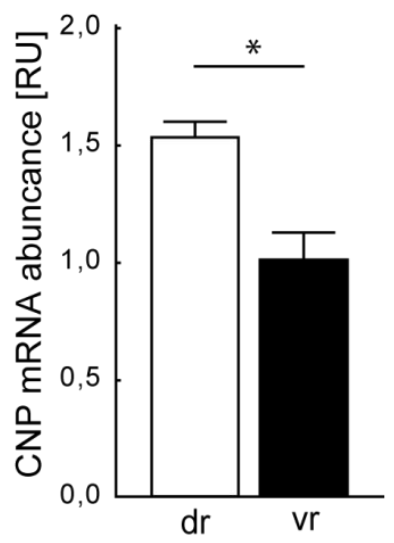

B

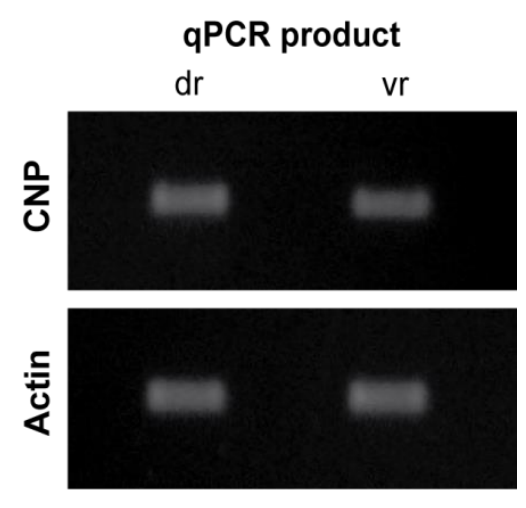

Fig 5: CNP transcript levels are elevated in dorsal roots

A: qRT-PCR analyses of dorsal and ventral roots of 6 months old wildtypes. Dorsal roots display $55 \%$ more CNP specific mRNA transcripts. ( $n=6$; Mean + SEM).

B: qRT-PCR products specific to CNP and Actin for dorsal and ventral roots visualized on agarose gels. Single bands proof specificity of the reactions.

\subsubsection{Nonmyelinating Schwann cells do not express substantial CNP levels}

Among sensory axons myelinated fibers are outnumbered by unmyelinated C-fibers (Ochoa and Mair, 1969). C-fibers are grouped and associated with nonmyelinating Schwann cells that engulf and separate the axons and thus form Remak bundles. As previously described, CNP is robustly expressed in Schwann cells that are associated with small caliber axons in dorsal roots. It has not explicitly been shown if adult nonmyelinating SC expressed CNP. Therefore, wax sections of wildtype sciatic nerves, which contain both, myelinated and nonmyelinated axons, were stained with the C-fiber marker Peripherin (orange; C-fibers and very small myelinated fibers), as well as CNP (green) to investigate overlapping staining patterns. Nonmyelinating SC of Remak bundles (borders of bundles outlined in white, only 
bundles with nucleus were considered) displayed no detectable CNP-staining in their perinuclear cytoplasm (Fig 6). This suggests that CNP expression levels in nonmyelinating SC are below the limits of detection or that they do not express CNP at all. This finding was important for the following experiments as it argues for a specific impact of CNP for myelinating Schwann cells surrounding small caliber axons. Moreover, pathological changes in CNP deficient nerves can thus be attributed to myelinating SC of small axons.

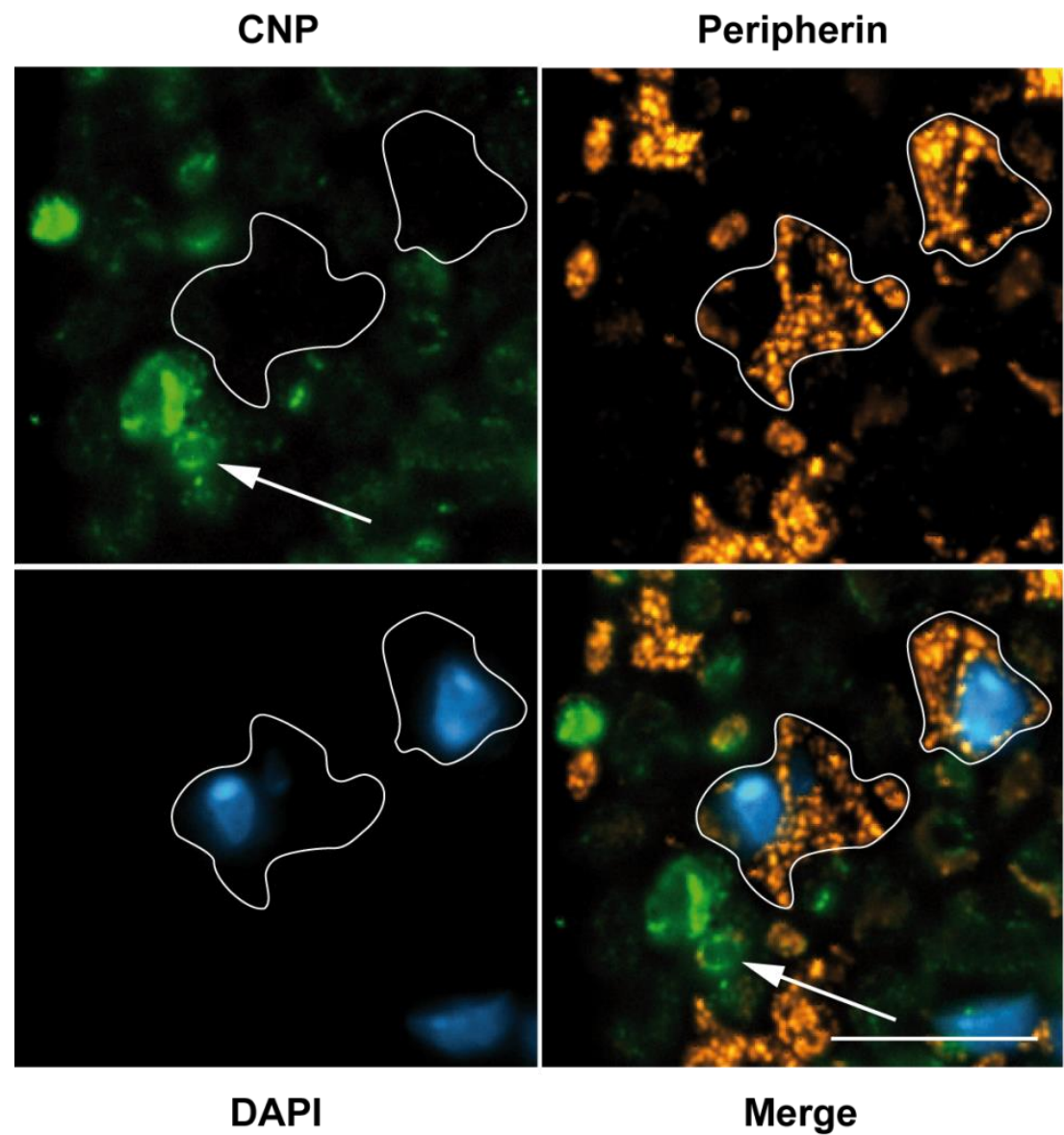

Fig 6: Nonmyelinating Schwann cells show no considerable CNP expression

Wax sections of sciatic nerves of 6 months old wildtypes immunofluorescently labeled for Peripherin (orange), which labels C-fibers, counterstained with CNP (green) and DAPI (blue, nuclear stain). No overlap of CNP and Peripherin-labeled Remak bundles can be observed. In contrast, myelinating SC associated with small caliber axons display a bright CNP staining (arrow), which serves as a positive control. (Scale bar represents $10 \mu \mathrm{m}$ ). 


\subsection{Ablation of CNP influences the noncompact myelin compartment}

Taken together, all observations point towards discrete Schwann cell populations with CNP being robustly expressed in myelinating glial cells that are in contact with small caliber axons. CNP deficient mice enabled us to investigate whether the impact of CNP function varies for small and large fibers as suggested by the different expression levels. It has been shown that CNP is localized to noncompact myelin areas as Schmidt-Lanterman incisures, paranodal loops, the inner and outer mesaxons as well as the cell bodies of Schwann cells (Braun et al., 1988; Trapp et al., 1988). CNP was described as a structural protein which is in intimate contact with the actin and tubulin cytoskeleton as well as with membranes (Braun et al., 1991; Bifulco et al., 2002; Lee et al., 2005). Furthermore, CNP has been shown to have an impact on inner tongue formation in the CNS (Edgar et al., 2009; Snaidero et al., 2014). Thus, we next addressed the morphology of the noncompact myelin compartment, where CNP is localized in the wildtype state. Analyses were mainly performed using saphenous nerves of wildtype and CNP deficient animals.

\subsubsection{Paranodal loops and Schmidt-Lanterman incisures remain unaltered}

Electron microscopic assessments of longitudinal saphenous nerve ultrathin sections did not show obvious alterations in the ultrastructure of paranodal loops (Fig 7A) or nodes of Ranvier nor in the structure of Schmidt-Lanterman incisures (SLI, Fig 7B). As it was difficult to judge the frequency of SLI on ultrathin nerve sections, further investigations on light microscopic level were carried out. To this end, teased sciatic nerve fibers were stained for the myelin associated glycoprotein (MAG; orange in Fig 7C) to visualize all incisures. To reliably recognize the dimensions of single internodes, fibers were costained for the voltage gated sodium channel Nav1.6 (green in Fig 7C) that labels nodes. The relation of SLIs numbers to the length of the axon was equal in CNP mutants and controls (Fig 7D). The thicker the fibers and their myelin, the more SLIs were observed in a given distance (compare different axonal sizes in Fig 7E). No differences were detected between wildtype and CNP deficient animals for any fiber type (Fig 7E). Moreover, internodal length measurements were included in this refined investigation of CNP deficient and wildtype nerve fibers. Therefore, teased fibers were stained for MBP (green in Fig 7F), which is expressed along the entire internode, as well as the paranodal protein Caspr (red in Fig 7F), that visualized the lateral ends of the internode. Internodal length measurements did not show any alteration, neither for small nor for large caliber axons. Thus, longitudinal dimensions of Schwann cells are not affected by the CNP deletion. 
A

\section{paranodal loops}

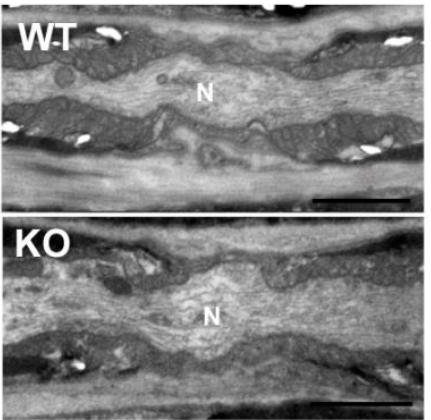

B

\section{Schmidt-Lanterman incisures}

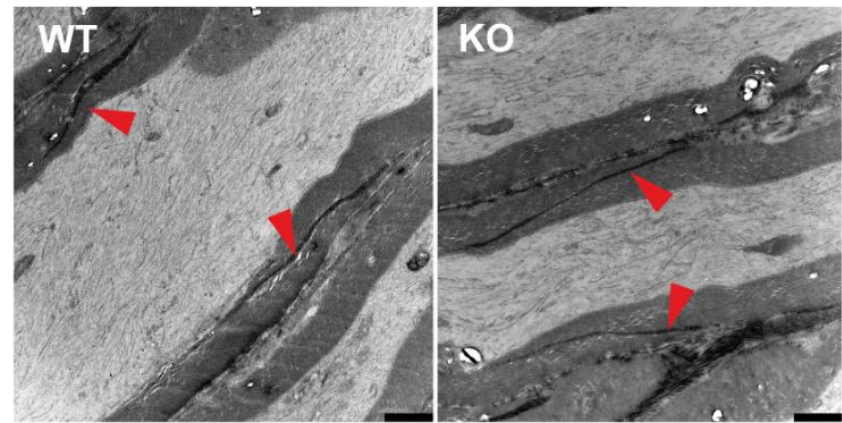

C
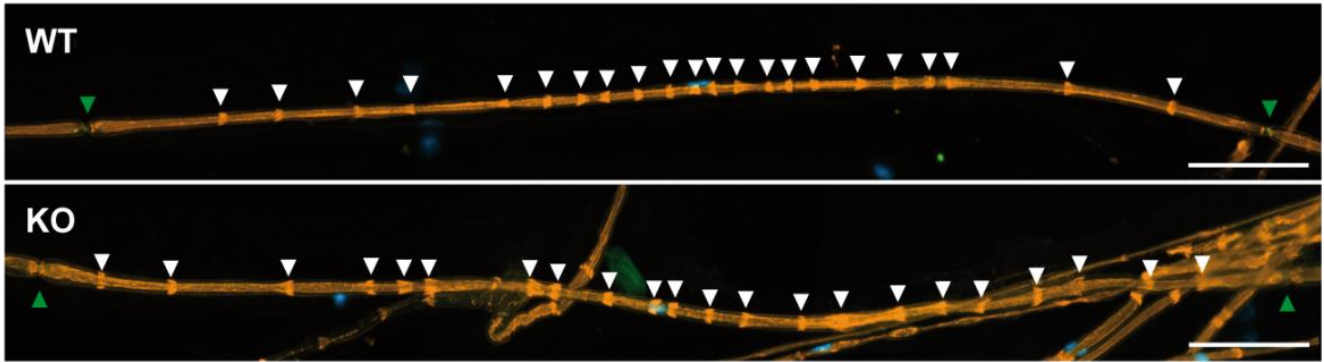

D

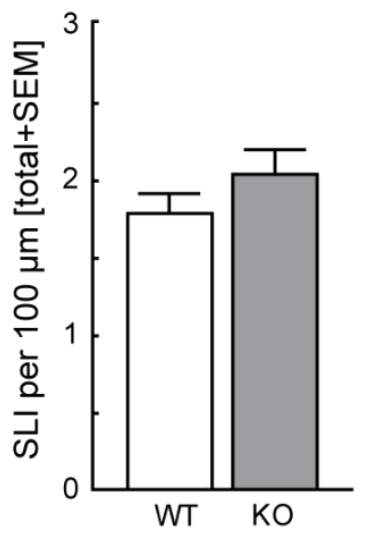

E

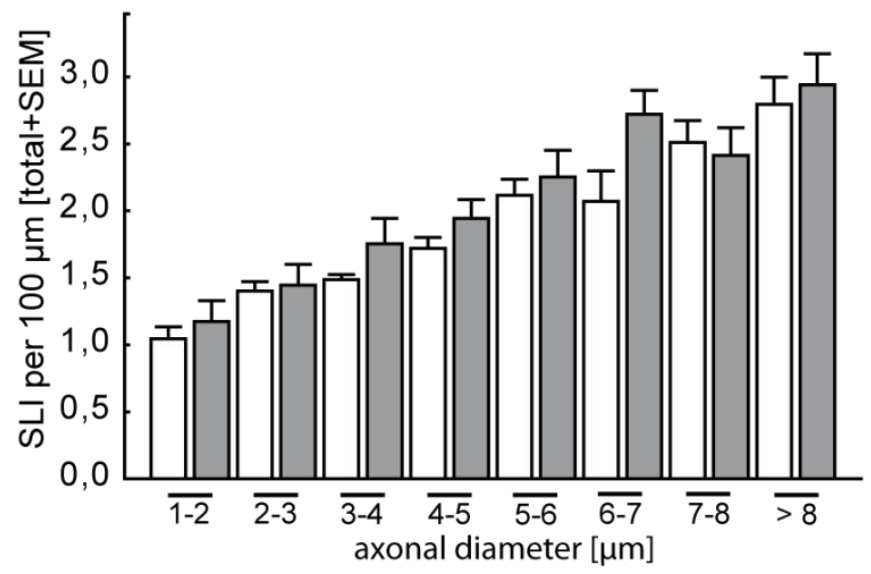

$\mathbf{F}$

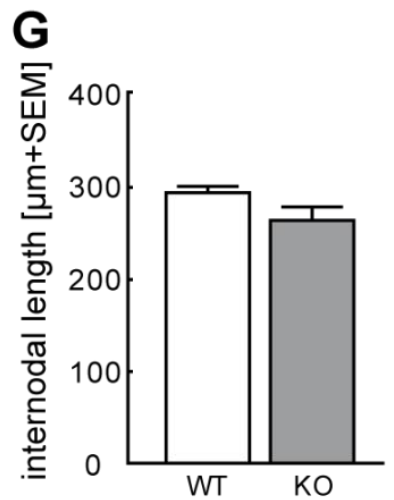

Fig 7: Unaltered paranodal loops and Schmidt-Lanterman incisures in CNP-/- animals

A: Electron micrographs of nodes with adjacent paranodal loops in longitudinal saphenous nerve ultrathin sections of 6 months old wildtype (WT) and CNP deficient animals (KO) display no differences. (Scale bars $1 \mu \mathrm{m}$ ). B: Schmidt-Lanterman incisures (red arrowheads) on electron micrographs of longitudinal ultrathin sections of saphenous nerve display a normal ultrastructure in CNP deficient animals (right) compared to wildtype controls (left) at 6 months of age. (Scale bars display $1 \mu \mathrm{m}$ ).

(Figure legend continued on the next page) 


\subsubsection{Inner tongues are smaller in the PNS of adult CNP deficient mice}

It has previously been shown that the deficiency of CNP causes swollen inner tongues in the central nervous system already at P5 (Edgar et al., 2009). Swellings of the inner tongue may interfere with the normal axon-glia interplay. High pressure frozen dorsal roots from CNP deficient and wildtype animals at 10 days, 1 month and 6 months of age were used to investigate inner tongue features in the PNS. Analyses were performed on small caliber axons because strongest alterations were expected for these fibers as they are associated with the Schwann cells that express most CNP in wildtypes. Inner tongues were categorized as small (left), normal (middle) or enlarged (right image) as illustrated in Fig 8A. Swollen inner tongues, as described for the CNS, were observed very rarely in the PNS. Unaltered frequencies of the three categories between WT and KO at postnatal day 10 possibly demonstrate that CNP function may only play a minor role in the PNS inner tongue formation during developmental myelination. However, in juvenile stages (1 month of age) CNP deficient mice displayed an increase in enlarged inner tongues, which reversed with age. Within dorsal roots of 6 months old animals the amount of enlarged inner tongues was significantly decreased whereas the number of axons with very small inner tongues increased. One possible explanation for the discrepancies between 1 and 6 months of age could be that fibers that showed enlarged inner tongues in early stages may be lost during adulthood. Another possibility would be that during late stages of active myelination the transport within the noncompact compartment might be hampered and material accumulates in the inner tongue (enlarged at $1 \mathrm{~m}$ ), which is slowly released when the sheath is established (smaller at $6 \mathrm{~m}$ ). However, at 6 months of age, when animals start to show symptoms (Lappe-Siefke et al., 2003) this specific part of the noncompact compartment was decreased which may directly influence the axon-glia communication.

\footnotetext{
Continued legend from figure 7

C: Teased sciatic nerve fibers from 6 months old wildtype and CNP deficient animals stained against MAG (orange, SLI, white arrowheads) and Nav1.6 (green, node, green arrowheads) as well as DAPI (blue, nuclei). (Scale bars represent $100 \mu \mathrm{m}$ ).

D: Quantifications of Schmidt-Lanterman incisures (MAG staining in C) along the corresponding internodes in teased fiber preparations of 6 months old CNP deficient (grey) and wildtype control animals (white) show no significant differences. (WT $n=4, K O n=6$; Mean + SEM).

E: All fibers summarized in $D$ have further been related to the axonal diameter. Similarly no group of fibers showed differences in the SLI spacing in CNP mutants compared to wildtypes. (WT $n=4, K O n=6$; Mean + SEM). F: Teased fiber preparation of a 6 months old wildtype animal stained for MBP (green, myelin), Caspr (red, paranode) and DAPI (blue, nuclei). Bottom image illustrates how measurements from paranode to paranode were performed (white lines) using ImageJ. (Scale bars represent $100 \mu \mathrm{m}$ ).

G: Quantification of the internodal length in wildtype (white) and CNP deficient animals (grey) reveals normal sized internodes also when CNP is ablated. $(n=5$; Mean + SEM).
} 
A
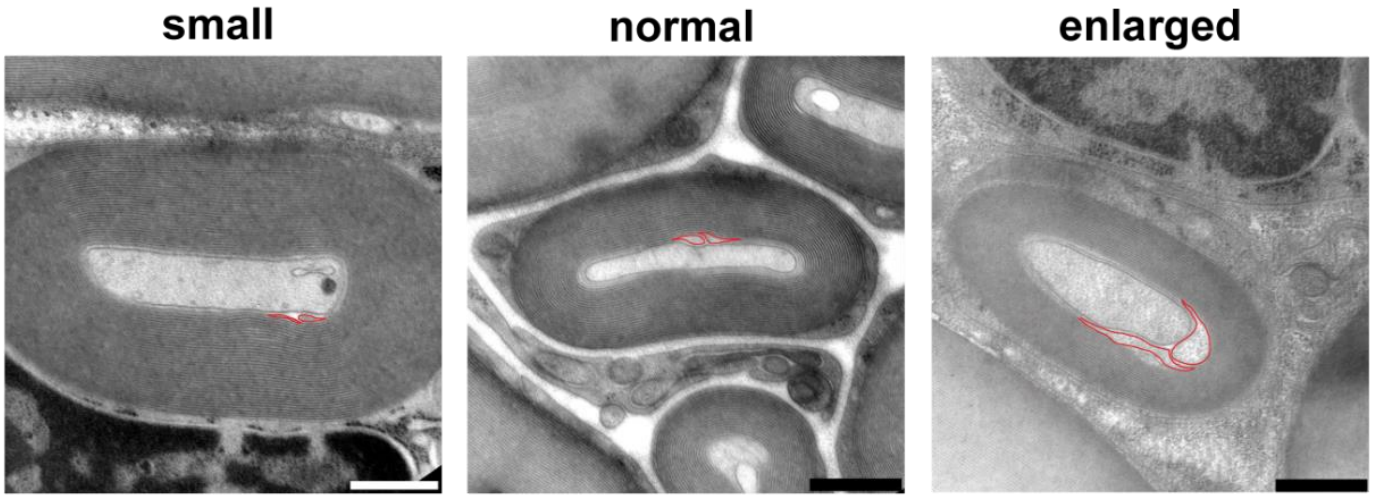

B

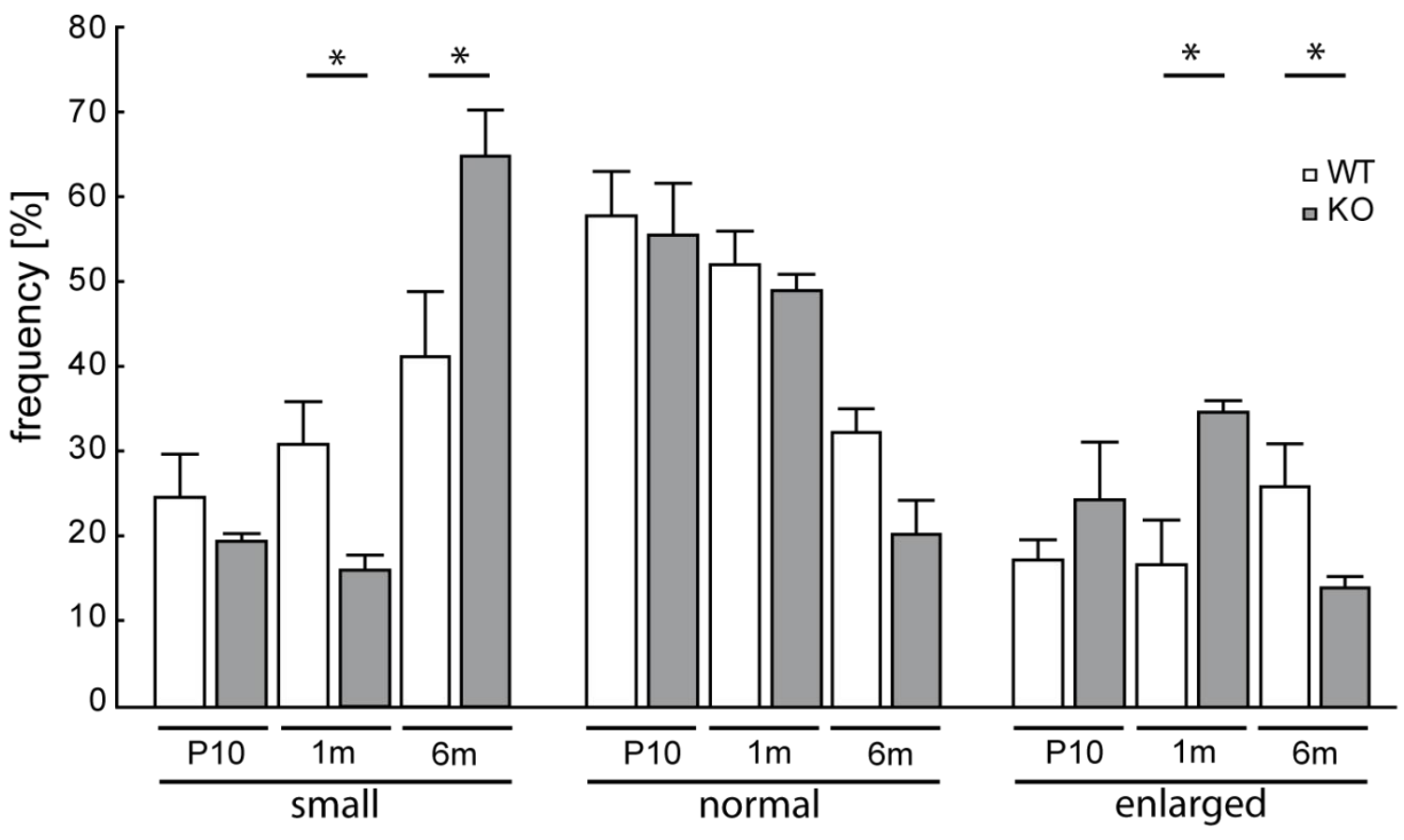

Fig 8: Smaller inner tongues in adult CNP mutants

A: Electron micrographs of high pressure frozen dorsal root axons of 1 month old animals illustrate small, normal and enlarged inner tongues of SC surrounding small caliber axons. (Scale bars display $500 \mathrm{~nm}$ ).

B: Frequency quantification of small, normal and enlarged inner tongues as illustrated in (A) for 10 days, 1 month and 6 months old CNP deficient (grey) and control (white) animals. The frequency of small inner tongues increases with age in both genotypes. CNP deficiency causes a further decrease of inner tongue size in adult animals (small inner tongues at 6 months in WT 41\% and KO 65\%; $n=4$; Mean + SEM). 


\subsubsection{Cytoplasmic channels are less frequent in adult CNP mutants}

Cytoplamic channels, another part of the noncompact myelin network in glial cells, have recently been reported to be reduced when oligodendrocytes lack CNP (Snaidero et al., 2014). The channels represent highly dynamic shortcuts through the compact myelin sheath, which occur in the peripheral nerves in addition to the more static Schmidt-Lanterman incisures. Therefore it is believed that they serve different functions. In peripheral nerves the number of cytoplasmic channels of 10 days old CNP deficient animals was not significantly different from that of wildtypes (illustrated in Fig 9A). In contrast, at 1 month of age a trend to a decreased amount of channels has been observed when the SC were devoid of CNP ( $p=0.07$ in Fig 9B). This decline becomes manifested during adulthood. Hence, small fibers in high pressure frozen dorsal roots of 6 months old CNP deficient animals showed cytoplasmic channels less than half as often as they were observed in small caliber axons in wildtype dorsal root samples (Fig 9B, right bars). Together with the reduced size of inner tongues, these data revealed that adult CNP deficient nerves show a further reduction within the noncompact myelin compartment in the PNS.

A

\section{cytoplasmic channel}

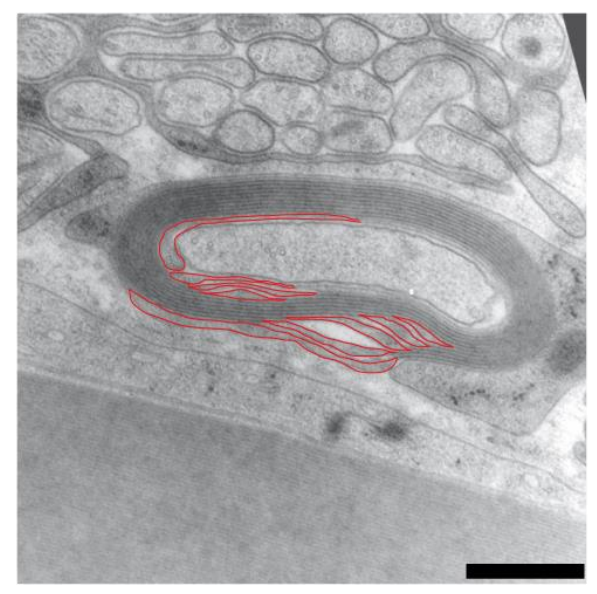

B

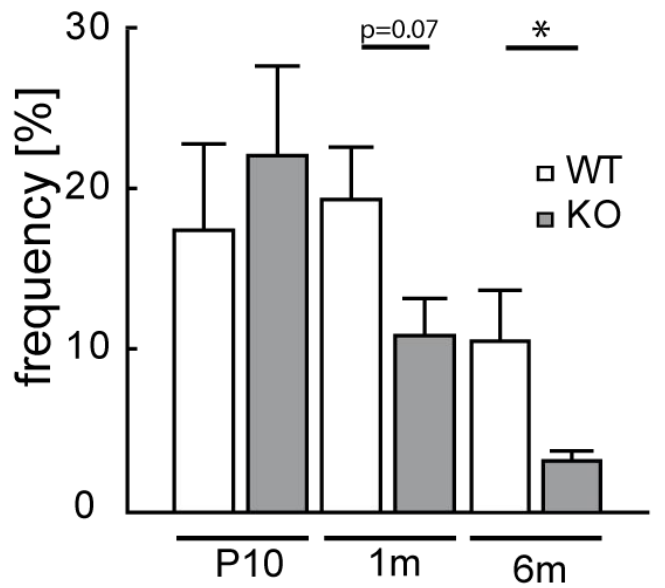

Fig 9: Cytoplasmic channels are less frequent in adult CNP mutants

A: Electron micrograph of a small caliber axon of a wildtype high pressure frozen dorsal root at 1 month of age displays a cytoplasmic channel through the compact myelin sheath (outlined in red). (Scale bar of $500 \mathrm{~nm}$ ).

B: Cytoplasmic channel quantifications for small caliber dorsal root axons of P10, 1 month and 6 months old CNP deficient (grey) and wildtype (white) animals reveal a decrease of cytoplasmic channels with age and further reduction when SC lack CNP ( $10 \%$ in WT and $3 \%$ for KO at 6 months; $n=4$; Mean + SEM). 


\subsubsection{The inner mesaxon is fully compacted in CNP mutants}

During CNS myelination the myelin sheath gets compacted towards the inner mesaxon finally leaving only the inner tongues filled with cytoplasm. When CNP is ablated this process happens faster (Snaidero et al., 2014). The actual process of compaction and the impact of CNP have not been investigated in the PNS so far. To this end analyses of the inner mesaxons of small caliber axons in high pressure frozen dorsal roots of CNP deficient and wildtype animals were performed. Surprisingly, there was no effect of CNP deficiency on the compaction states of the innermost myelin layer at postnatal day 10 (Fig 10), when CNS axons showed striking differences. At one month of age the frequencies of fully and partially compacted inner mesaxons still did not show a difference between the genotypes. On the other hand, SC that form a complete ring of cytoplasm in the innermost myelin layer (right image in Fig 10A) have more frequently been seen in CNP deficient animals compared to controls at one month of age. In contrast, at 6 months of age fibers with a whole cytoplasmic ring were observed equally seldom in controls and mutant animals. In both genotypes a strong increase of fibers with fully compacted inner myelin layer was observed compared to younger time points. Simultaneously, the proportion of small fibers that still contained cytoplasm in the inner myelin layer was considerably reduced when animals aged. Interestingly, at the age of 6 months a significant difference was further detected between CNP deficient and control animals. Schwann cells lacking CNP had more often a completely compacted inner myelin sheath, which goes along with a reduced occurrence of fibers that still contained cytoplasm (Fig 10B). Within the CNS an increased compaction speed has been described during development (Snaidero et al., 2014) which was not detectable in the PNS at young stages. However, an increased state of compaction was reached in peripheral nerves of CNP deficient animals in adulthood. Together, the increased compaction in the CNS and PNS suggests that CNP may equally act as spacer in oligodendrocyte and SC myelin. In summary the refined analyses of all noncompact myelin domains of small caliber peripheral fibers revealed a reduction of the compartment when CNP is absent. This may compromise transport processes within the SC and possibly deteriorates axonal support. 
A
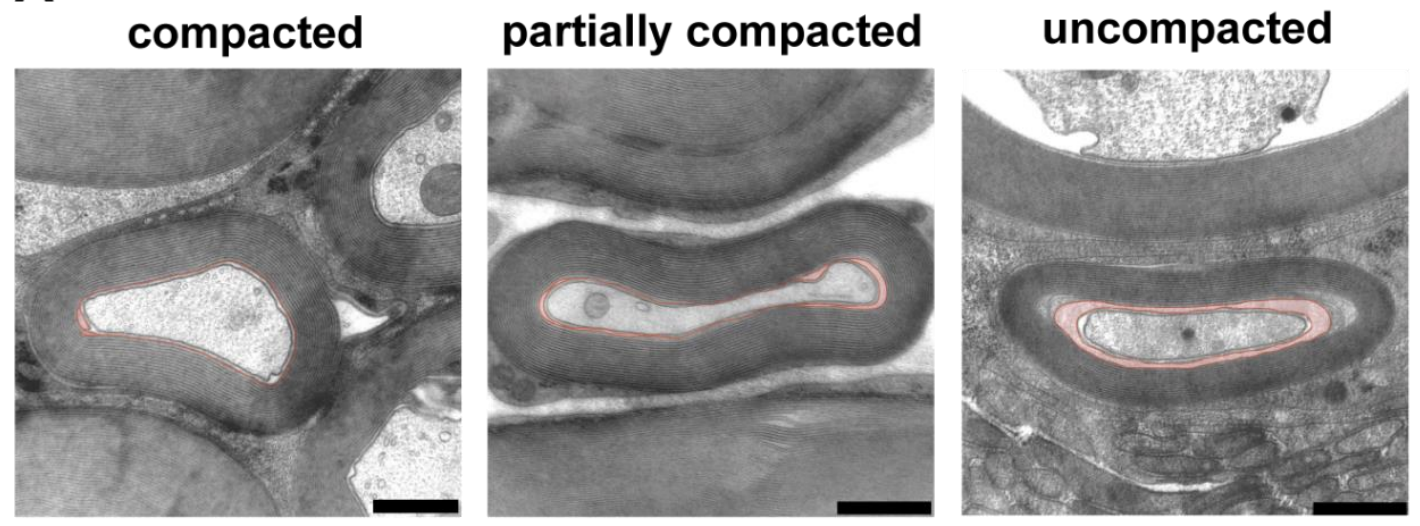

B

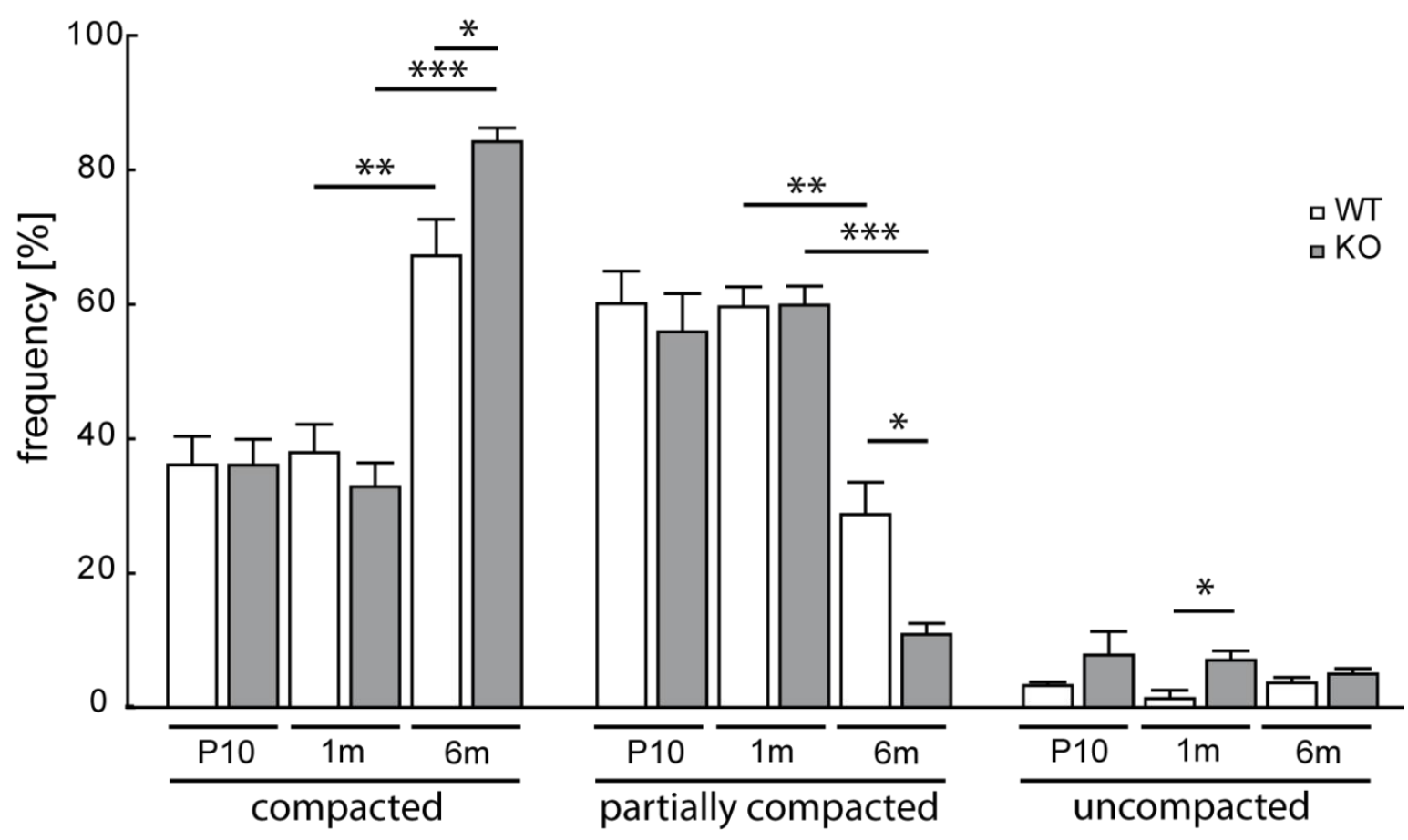

Fig 10: The inner mesaxon is more compacted in CNP deficient animals

A: Electron micrographs of high pressure frozen small caliber dorsal root axons of 1 month old animals depict inner mesaxons which are fully "compacted", that contain some cytoplasm (partially compacted) or that form a whole cytoplasmatic ring (uncompacted) underneath the compact myelin sheath. (Scale bars display $500 \mathrm{~nm}$ ).

B: Quantification of the frequencies of the three categories illustrated in (A) of P10, 1 month and 6 months old CNP deficient (grey) and control (white) animals display an increase in compaction with age and additionally when SC lack CNP. (from $38 \%$ in WT at 1 month to $67 \%$ for WT at 6 months and further up to $84 \%$ in $\mathrm{KO}$ at 6 months; $n=4-5$; Mean + SEM). 


\subsection{CNP deficiency causes hypermyelination of small fibers}

The absence of the noncompact myelin protein CNP provokes changes within its compartment also in the PNS. Even though the compaction speed during central myelination was altered upon CNP deficiency (Snaidero et al., 2014) the myelin sheath thickness was found to be normal in the central nervous system (Lappe-Siefke et al., 2003). We therefore next questioned if this can equally be observed in the PNS or if the compact myelin would follow the decrease of the noncompact myelin to keep the ratio between the two compartments.

\subsubsection{Adult onset hypermyelination of peripheral nerves in CNP mutant mice}

Surprisingly, on light microscopic level (not shown) and more clearly on electron micrographs of sensory peripheral nerves an increased myelin sheath thickness was detectable as illustrated in Fig 11A. Quantifications of the g-ratio (the relation between axonal diameter and the respective myelinated fiber diameter) within saphenous nerves of 6 months old CNP mutant mice revealed a significant reduction compared to wildtype control animals of the same age. Thus, against our expectations, on average the myelin sheaths were thicker in peripheral nerves of adult CNP deficient mice (Fig 11B). Investigations of different axonal subpopulations (small compared to large caliber axons) further uncovered that small caliber fibers displayed the most pronounced hypermyelination when CNP was ablated. On the other hand, fibers above $6 \mu \mathrm{m}$ in diameter did not exhibit differences in their myelin sheath thickness anymore (Fig 11C right column). Even though axonal loss in the CNS has been shown to a small extent already in young animals (P5 in spinal cord; Edgar et al., 2009), CNP deficient mice appeared normal during early life but became severely affected with age (Lappe-Siefke et al., 2003; Wieser et al., 2013). To determine hierarchies of pathological changes younger animals needed to be investigated as well. To this end, the myelin sheath thickness was also quantified at postnatal day 5 as well as at 1 month of age. At P5 the myelin sheath thickness of CNP deficient animals was not significantly different from that of wildtype controls, even though a trend towards decreased g-ratios was already visible. In 1 month old animals this trend reached significance for the smallest fibers, whereas the other fibers remained to display only trend towards an increased myelin sheath thickness. This means that the difference seen at 6 months of age developed in later life, when myelination would normally be finished. Hence, the sheath may fail to stop growing upon CNP deficiency. 


\subsubsection{Unaltered myelin periodicity in CNP deficient animals}

An increase in myelin sheath thickness can either be caused by an alter compaction or by an accumulation of additional myelin wraps. To better understand the CNP function it needed to be discriminated between the two possibilities. Therefore, the spacing of the myelin layers of small hypermyelinated (1-2 $\mu \mathrm{m}$ in diameter) saphenous nerve axons of CNP deficient animals was compared to that of wildtypes. The periodicity of the myelin lamellae was found to be of wildtype level also in CNP deficient mice (Fig 11D). This implies that the hypermyelination described in Fig 11A-C was caused by an addition of ultrastructurally normal myelin wraps throughout adult life. Particularly SC associated with small caliber axons thus produced tremendously more myelin membranes when CNP was ablated. This further suggests a role of CNP as a myelination break or an adult negative regulator of myelination. To rule out, that the observed relative increase in compact myelin is not just secondary to a reduction in axonal diameters at constant myelin levels, the fiber diameters themselves were investigated. The average axonal diameter in 6 months old CNP mutant mice was not altered compared to the mean fiber size of wildtype control animals at the same age (Fig 11E).

\subsubsection{Small motor fibers are equally affected}

All aforementioned investigations of myelination states were performed on saphenous nerves, which contain predominantly sensory fibers. To exclude an impact of the actual nerve characteristic (motor or sensory) on the myelination status during CNP ablation, similar investigations were performed for ventral roots which contain purely motor fibers. A considerable increase in myelin sheath thickness was also observed for motor fibers of 6 months old mutant mice (Fig 11F). G-ratio measurements of different axonal subpopulations within ventral roots revealed hypermyelination of small motor fibers just as it was seen for small sensory fibers in saphenous nerves of CNP deficient animals (Fig 11F diagram). Hence, the hypermyelination is not dependent on the axonal identity but only correlates to the diameter of the enwrapped fiber. Taken together, small caliber axons are hypermyelinated when SC lack CNP. With regard to the reduced noncompact compartment as shown in section 4.3 the imbalance between the two compartments is even amplified by the hypermyelination. This may further impair SC intrinsic and axon-glial transport processes and could thus deteriorate axonal support for small fibers. 
A

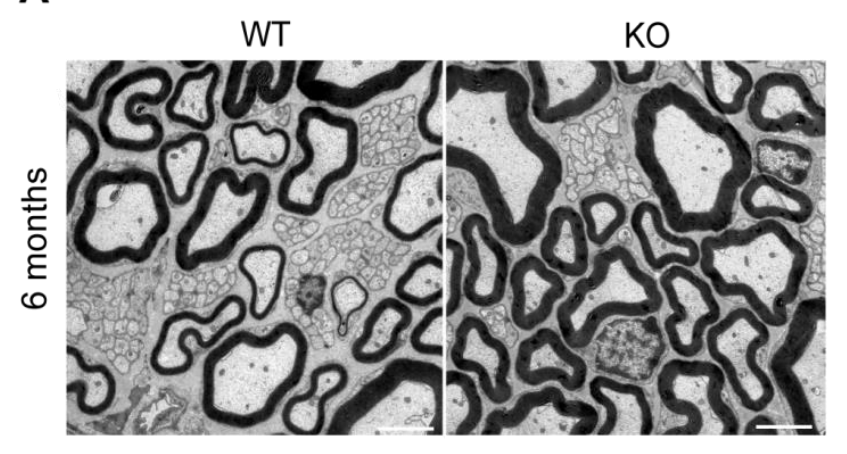

C
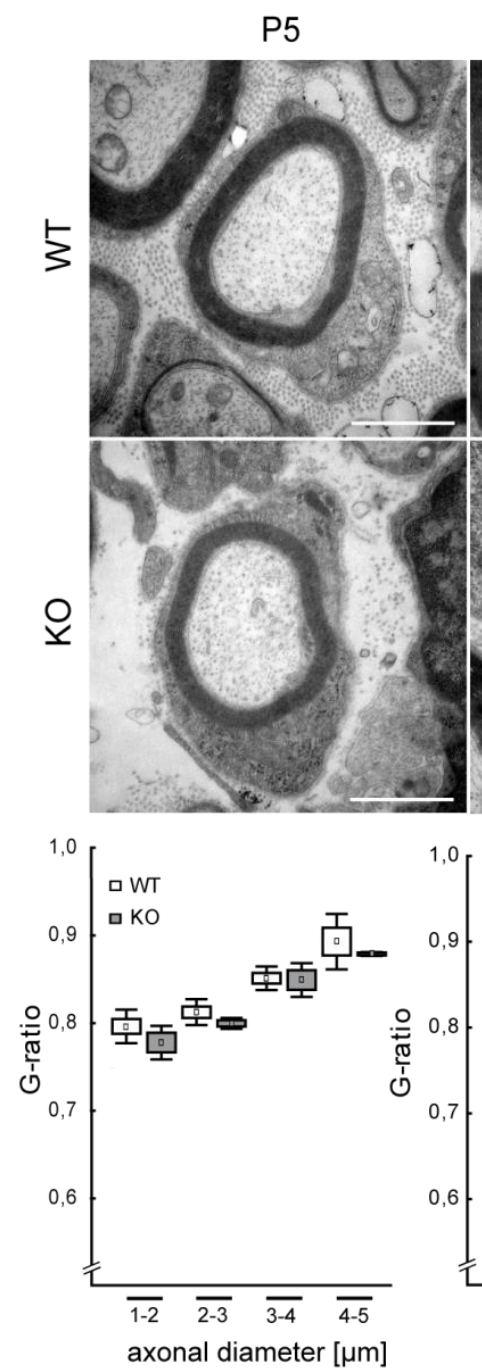

D

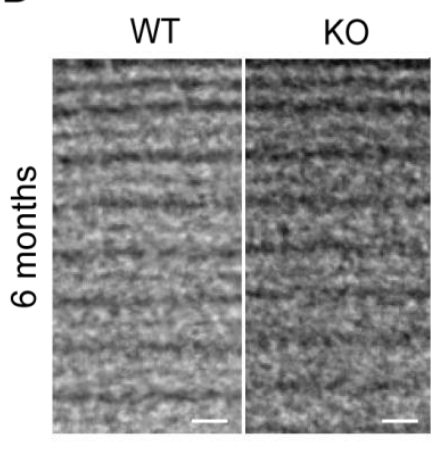

1 month
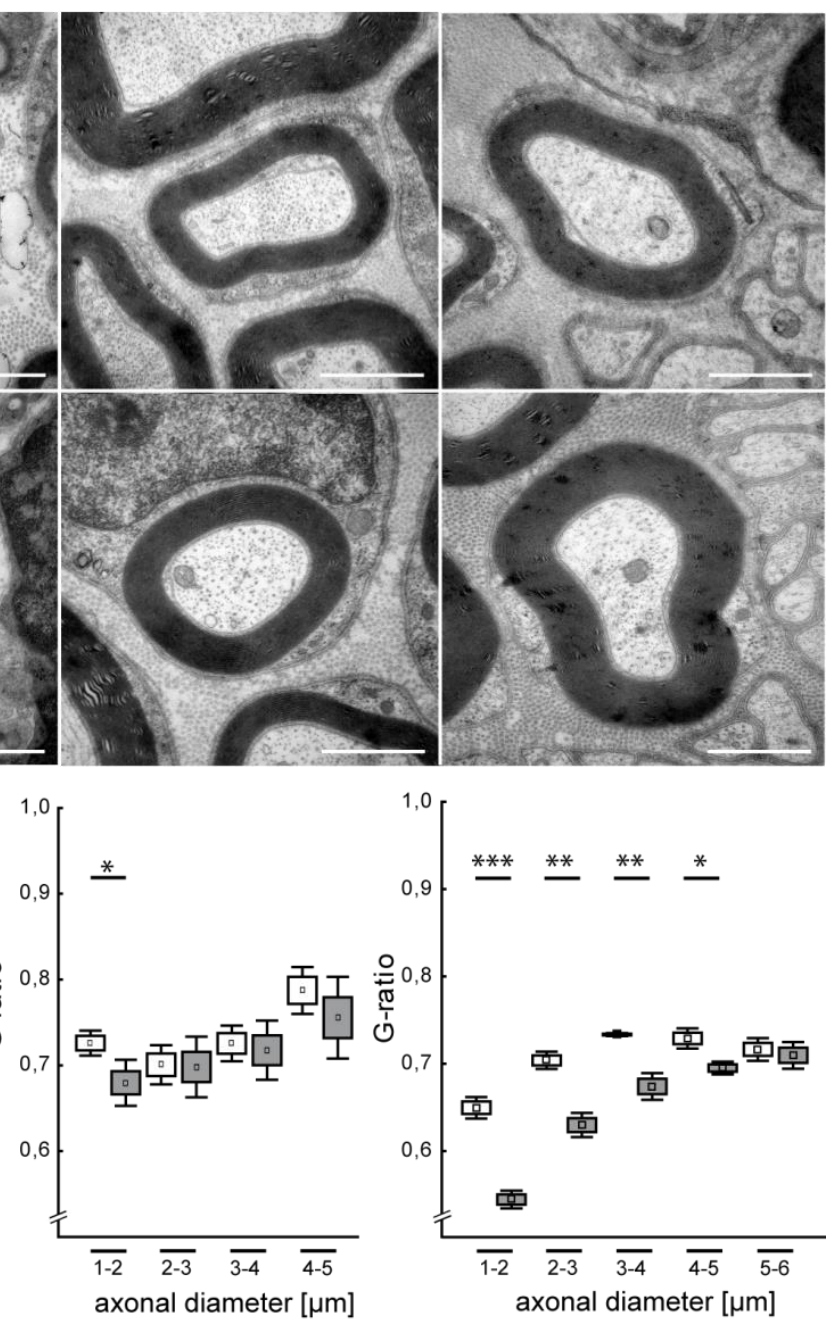

B

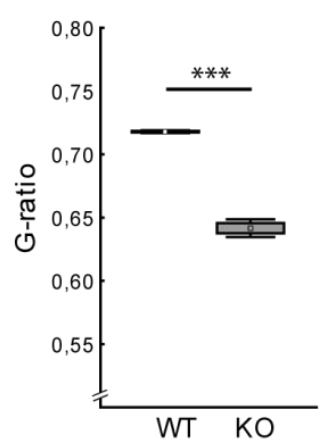

6 months

E

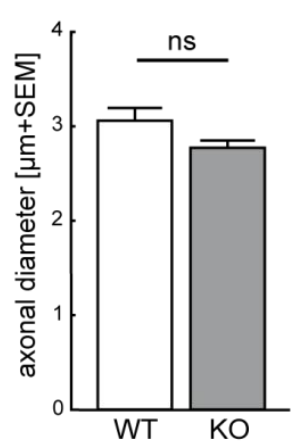





Fig 11: Hypermyelination of small caliber axons in CNP deficient mice

A: Overview electron micrographs of saphenous nerves of 6 months old CNP deficient and wildtype animals to demonstrate the increase in myelin sheath thickness of small caliber axons. (Scale bars of $2.5 \mu \mathrm{m}$ ).

B: Quantification of the mean myelin sheath thickness as ratio of axonal diameter divided by myelinated fiber diameter (g-ratio) for wildtype (white) and CNP-/- animals (grey) at 6 months of age. Mutant mice show a decreased $g$-ratio which reflects thicker myelin. ( $n=3$ per group; Mean, SEM and SD).

C: Representative electron micrographs of small caliber saphenous nerve axons from wildtype and mutant animals at P5, 1 month and 6 months of age. g-ratios were related to the respective axonal diameter. Both genotypes show an increase of the myelin thick thickness with age. The myelin sheath thickness is further increased in small to mid-sized fibers in CNP mutants at 6 months of age. (P5 n=5/3, 1 month $n=3 / 4,6$ months $\mathrm{n}=3$; Mean, SEM and SD; Scale bars depict $1 \mu \mathrm{m}$ ).

D: High magnification micrograph of myelin lamellae around small caliber axons from wildtype and CNP deficient saphenous nerves. The myelin periodicity is unaltered. ( $n=3$ per group; Mean + SEM; Scale bars of $10 \mathrm{~nm}$ ).

E: Axonal diameter quantification of saphenous nerves from 6 months old mutant (grey) and wildtype (white) animals display no significant difference. ( $n=6$ per group; Mean diameter from perimeter + SEM).

F: High magnification electron micrographs of small caliber ventral root fibers of wildtype and CNP deficient animals illustrate that small motor axons are also hypermyelinated. Myelin sheath thickness quantification relative to the axonal diameter displays a decreased g-ratio in CNP deficient (grey) animals which is more pronounced the smaller the fibers are. (Scale bars of $1 \mu \mathrm{m} ; \mathrm{n}=3$; Mean, SEM and SD). 


\subsubsection{No evidence for focal hypermyelination or myelination threshold shift}

In many hypermyelinating mouse mutants for example in Dlg1, PTEN or PMP22 mutants (Cotter et al., 2010; Goebbels et al., 2012; Adlkofer et al., 1995) one special feature is the appearance of focal hypermyelination. During all electron microscopic assessments of CNP deficient mice and wildtype controls for this work myelin tomacula and inclusions, as well as outfoldings were observed very rarely. When quantifying their frequency no significant difference was detectable between CNP deficient mice and wildtype controls (Fig 12A-B). Apart from focal hypermyelination some glial mutations cause sorting defects and myelination threshold shifts which for instance mean, that fibers smaller than $1 \mu \mathrm{m}$ in diameter are sorted and myelinated during development (Guo et al., 2013; Taveggia et al., 2005). Saphenous nerve investigations in 6 months old CNP mutants and respective controls did not show differences in the threshold axonal size for myelination (Fig 12C). That distinct CNP-loss-of-function phenotype compared to other hypermyelinating mouse models imposes different underlying mechanisms that trigger the increased myelin growth.

A
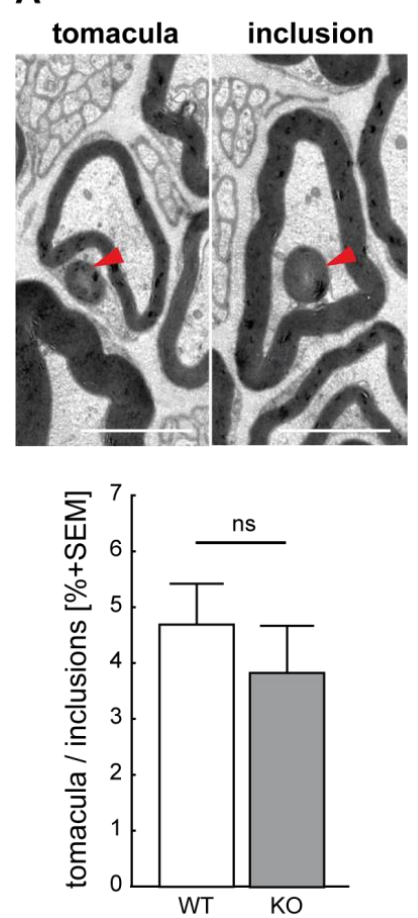

B
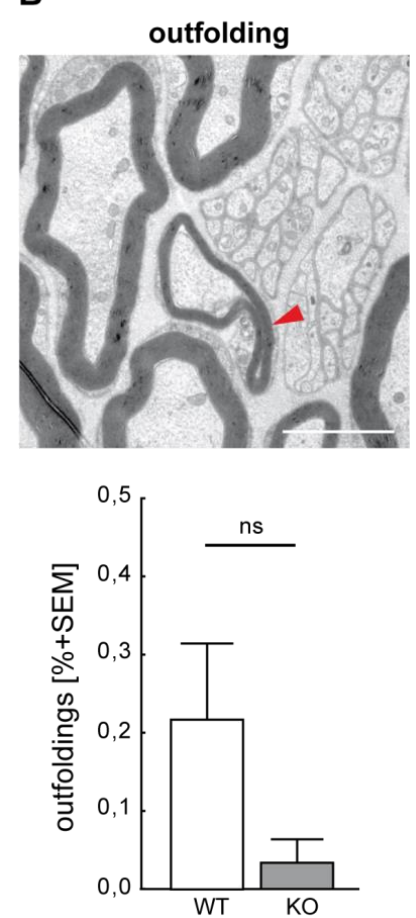

C
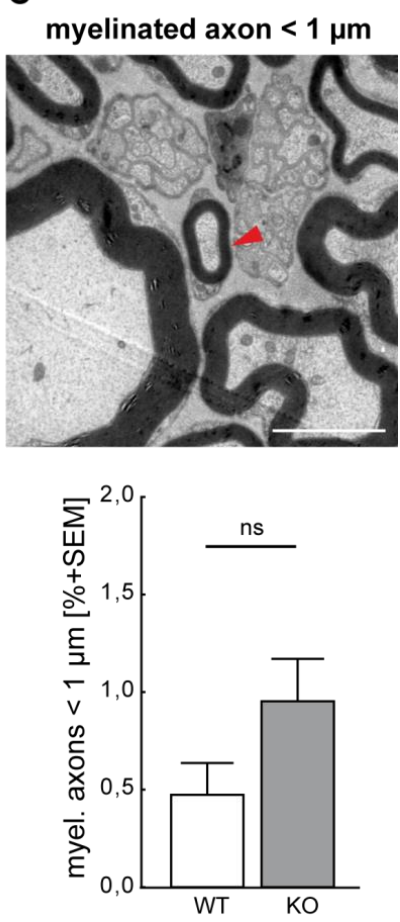

Fig 12: No focal hypermyelination and unaltered myelination threshold

A: Electron micrographs of myelin tomacula (left) and myelin inclusions (right), that can be observed as frequently in saphenous nerves of 6 months old CNP-/- (grey) animals as in wildtype controls (white) of the same age (related to total axonal counts). ( $n=6$; Frequency + SEM; Scale bars depict $2.5 \mu \mathrm{m}$ ).

B: Representative electron micrograph of a myelin outfolding (arrowhead). Frequency quantifications show no significant differences between wildtype (white) and CNP deficient animals (grey) at 6 months of age. Outfoldings are only very rarely visible and especially in the wildtype group a high variability in their frequencies is observed. $(n=6$; Frequency + SEM; Scale bar of $2.5 \mu \mathrm{m})$.

C: Electron micrograph of a myelinated axon below $1 \mu \mathrm{m}$ in diameter. Quantifications of these fibers show no significant changes in the myelination threshold in CNP deficient mice at 6 months of age (grey) compared to age matched wildtype controls (white). ( $n=6$; Frequency + SEM; Scale bar depicts $2.5 \mu \mathrm{m}$ ). 


\subsubsection{Molecular alterations caused by the CNP deficiency}

The most remarkable effect on peripheral nerves of CNP deficient mice was the hypermyelination of small caliber axons. To unravel underlying mechanisms responsible for the ongoing myelination in adult life, peripheral nerves were further investigated on molecular level.

\subsubsection{No obvious alterations of the transcriptional control of myelination}

CNP overexpressing mouse mutants show a shifted peak of myelin gene expression. They display highest levels of myelin protein specific mRNA transcripts at two weeks of age compared to the normal peak at 3 weeks in control animals (Gravel et al., 1996). On the other hand, CNP deletion may delay the peak in myelin gene expression out of the critical myelination window. This might impair the end of myelination and could therefore explain the continous myelination especially in sensory nerves. To test this hypothesis, myelin specific RNA profiles were generated in a timeline from postnatal day 5 to 12 months of age for dorsal and ventral root tissue (Fig 13). In general, CNP specific mRNA levels as well as those of PLP were found to be higher in dorsal compared to ventral roots. Other myelin specific transcripts (PO, MBP and MAG) on the other hand showed relatively equal abundances. Against expectations CNP deficient tissue did not show obvious alterations of the RNA profiles compared to wildtype nerve samples. Only MBP and MAG transcripts were slightly but not significantly increased in mutant nerves. None of the tested genes showed a shifted peak of expression. Especially after one month of age when additional myelin is accumulated in CNP deficient animals no differences were observed. This suggests that the hypermyelination provoked by CNP deficiency is caused posttranscriptionally.
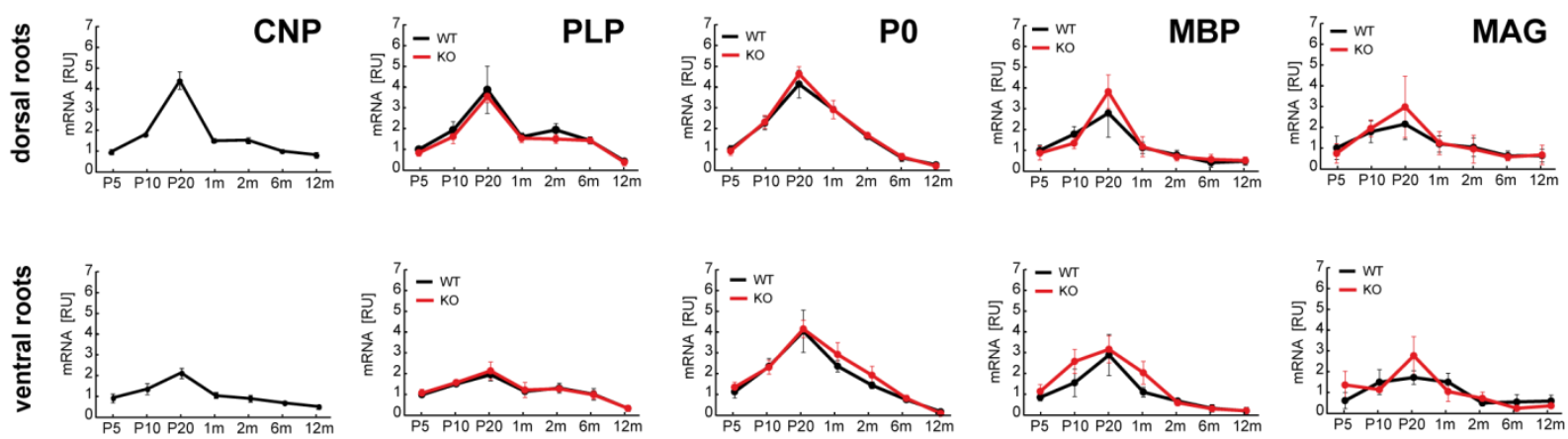

Fig 13: Myelin specific mRNA profiles are unaltered when CNP is ablated

Dorsal (upper row) and ventral root (lower row) mRNA profiles for CNP, PLP, P0, MBP and MAG in wildtype animals (black) and CNP mutants (red). Animals were analyzed at P5, P10, P20, P30, 2 months, 6 months and 12 months of age. The peak in myelin gene expression can be observed at 3 weeks of age in mutants and controls. No significant differences of RNA expression levels can be detected at any time point between wildtype and CNP-/- mice. ( $n=4$ per group and time point; Mean + SEM). 


\subsubsection{CNP deficient mice display activated signaling pathways}

The MEK/ERK and PI3K/AKT pathways are known to influence myelination especially by translational control. Alterations within these signaling pathways can provoke myelination defects in either direction (Taveggia et al., 2005; Newbern et al., 2011; Ishii et al., 2013; Sheean et al., 2014). Investigation of these pathways within CNP deficient mice on immunoblot level revealed alterations for both pathways. To gain insight into the activity state of the signaling pathways the ratio between phosphorylated and unphosphorylated protein isoforms has to be considered. However, during development (1 month of age) only minor changes in phosphorylated protein isoforms relative to the unposphorylated protein levels were observed for MAPK and AKT (Fig 14A). Contrary, immunoblot analyses of adult animals (6 months of age) revealed that both pathways show an increase in their active isoforms PMAPK and PAKT relative to the unphosphorylated proteins in dorsal roots of CNP deficient animals compared to wildtype controls (Fig 14B). Single pathway activation may induce demyelination and dedifferentiation of SC as shown for adult Raf activation (MAPK pathway; Napoli et al., 2012). Interestingly, the ratio of both pathways (MAPK signaling / AKT signaling) remained unaltered as relative levels of phosphoproteins increased similarly (Fig 14B right diagram) in dorsal roots of 6 months old CNP deficient animals. The balanced upregulation of both pathways probably prevents disadvantageous effects that have been observed if only one pathway was deregulated. Instead, it may drive the ongoing myelination.

\subsubsection{Increased expression of myelin specific and lipid related proteins}

The balanced increase in MAPK and AKT signaling in adult sensory nerves potentially caused enhanced myelin production in a developmental fashion. Indeed, myelin basic protein (MBP) as well as peripheral myelin protein 2 (MyP2) were found to be increased upon CNP deletion in adult dorsal roots as shown on immunoblots in Fig 15A. Apart from their specific protein constitution, myelin membranes are especially rich in lipids. Additionally, qRT-PCR analysis revealed an increase of $\mathrm{HmgCoA}$-reductase, the key regulator of lipid synthesis (Basson et al., 1988), and SCAP, which is involved in regulation of lipid transport (Verheijen et al., 2009), on mRNA level in dorsal roots of CNP mutant mice (Fig 15B). Together, this data pointed to enhanced myelin protein and lipid production in adulthood when Schwann cells are devoid of CNP. It has to be further investigated if this induction of myelin protein and myelin lipid production is actually downstream of the increased MAPK and AKT activity.

Taken together, CNP deletion has no impact on transcriptional regulation of myelination and may therefore cause posttranscriptional alterations within the Schwann cells. However, CNP ablation activated MAPK and AKT signaling which may enhance adult myelin production resulting in hypermyelination of small caliber axons. 
A

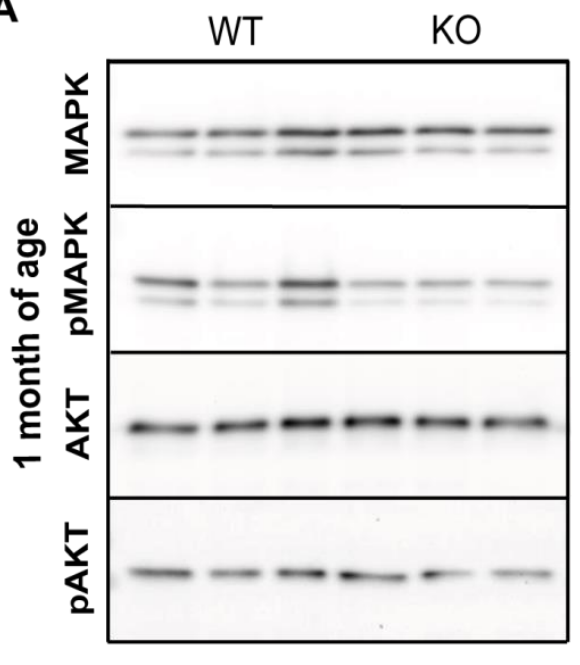

B

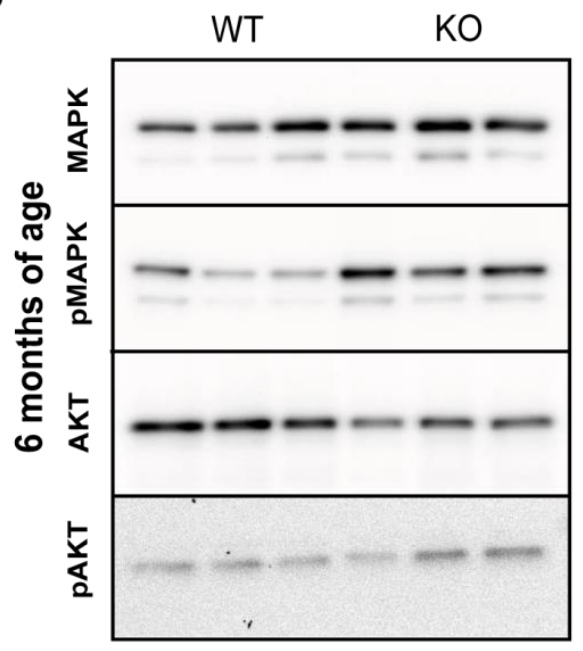

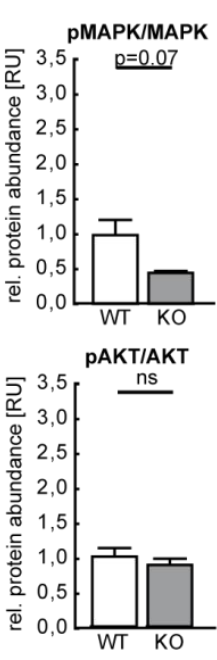
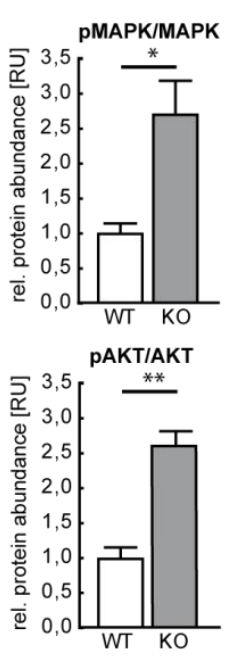

ratio of MAPK and AKT signaling

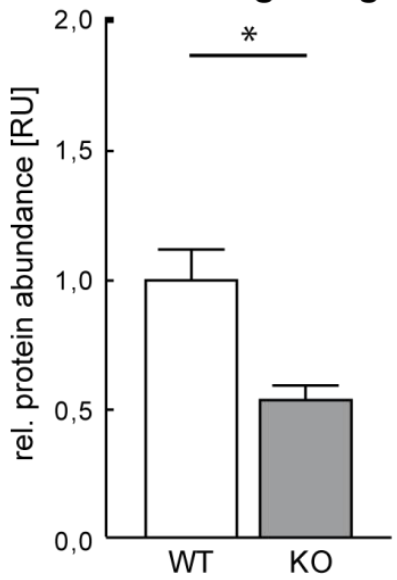

ratio of MAPK and AKT signaling

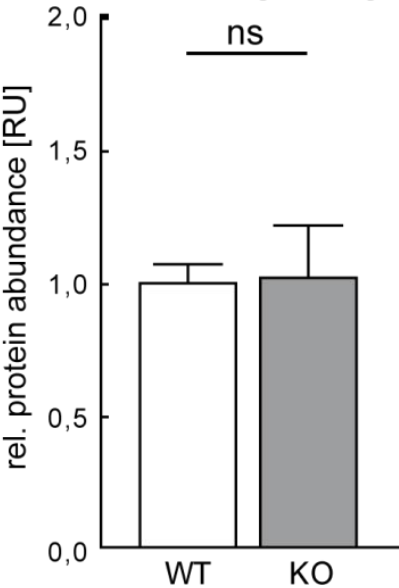

\section{Fig 14: Activation of MAPK and AKT signaling in CNP deficient nerves}

A: Immunoblots specific to MAPK and AKT and their respective phosphorylated isoforms from dorsal root samples of wildtype and CNP deficient animals at 1 month of age. Quantifications of the protein abundances display a trend to less activated MAPK signaling in CNP deficient animals (grey) compared to wildtype mice (white), whereas no difference is detectable for AKT and pAKT. ( $n=3$; Mean + SEM).

B: Similar immunoblots as in A for dorsal roots of 6 months old CNP deficient (grey) and wildtpye animals (white). Analyses of the phosphorylated isoforms related to the abundance of the non-phosphorylated proteins reveal a 2.5 -fold upregulation of PMAPK and PAKT levels. The ratio between the pathways remains unchanged (right diagram). $(n=3 ;$ Mean + SEM). 
A
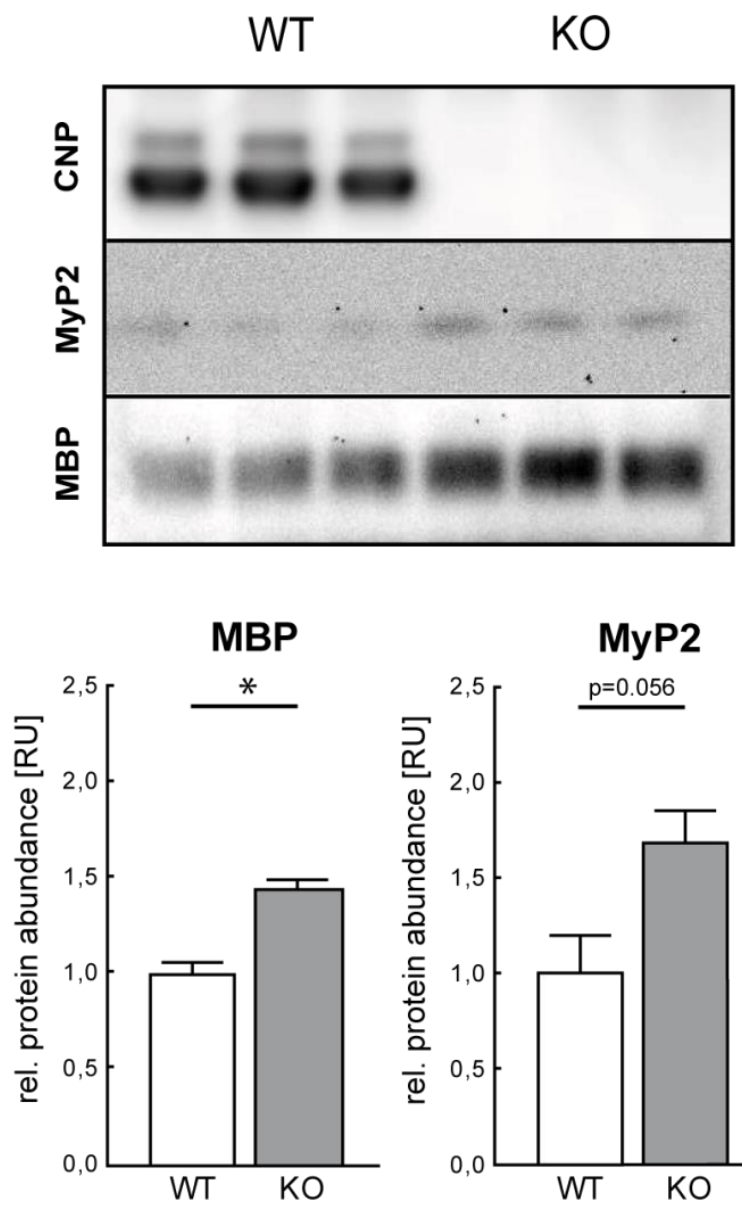

MyP2

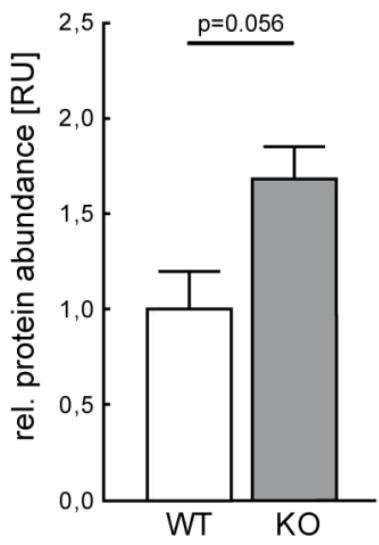

B
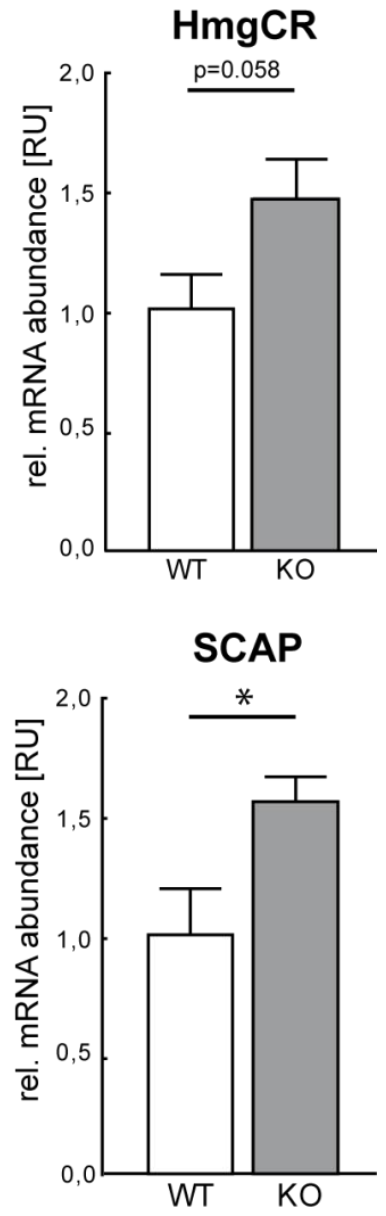

Fig 15: Increase of myelin proteins and lipid related genes in adult CNP-/- mice

A: Immunoblot analyses of myelin proteins CNP, MyP2 and MBP from dorsal roots of wildtype and CNP deficient mice. Quantifications reveal around $50 \%$ increased protein levels in 6 months old CNP mutants (grey) compared to wildtype controls (white). ( $n=3$; relative values + SEM).

B: Quantitative RT-PCR data of 6 months old CNP-/- (grey) and wildtype (white) animals display about $50 \%$ higher transcript levels of HmgCoA-reductase and SCAP in mutant mice. (WT n=6, KO n=7; Mean + SEM). 


\subsection{Impact of CNP deficiency for small caliber axons}

All aforementioned observations specifically point to an impact of CNP function for Schwann cells that engulf small caliber axons. Especially the proportion of noncompact and compact myelin was strikingly altered for small caliber fibers. In adult life CNP deficient animals displayed a reduction of the noncompact myelin compartment which hints to narrowed transport routes. Additionally, an increase in compact myelin was observed, which further enhances the imbalance of the two compartments. It was further investigated if the pathological alterations within the SC secondarily impair the integrity of underlying axons.

\subsubsection{Adult onset loss of small caliber axons}

During development myelinating Schwann cells enwrap and myelinate peripheral axons. Furthermore, it has been proposed that they trophically and metabolically support their underlying axons. As already described, CNP deficiency particularly led to changes in Schwann cells associated with small caliber axons. We next asked if these alterations within the SC influence the axonal integrity. When analyzing the quantity of myelinated axons within several peripheral nerves in adult animals (6 months of age), it became obvious that during adulthood CNP deficient animals displayed reduced numbers of intact myelinated fibers. The mixed sciatic nerve showed only slightly reduced fiber numbers (4\% decrease in Fig 16A). In contrast, the predominantly sensory saphenous nerve (7\% decrease in Fig 16C at 6 months) as well as the purely sensory dorsal roots (10\% decrease in Fig 16B left) displayed more striking differences. However, no alteration of myelinated fiber numbers were observed for the purely motor ventral roots (Fig 16B right). To relate the axonal damage to the alterations within the SC younger animals had to be analyzed. Time course experiments on saphenous nerves of wildtype and CNP deficient animals from postnatal day 5, up to 6 months of age revealed differences only for the 6 month time point (Fig 16C), suggesting that the axonal loss appeared secondary to changes within the SC which were already existing at 1 month of age. To unravel if a specific axonal subpopulation is prominently lost, axonal frequency quantifications on saphenous nerves of 6 months old animals were carried out. The frequency plots did not show differences regarding the axonal size distribution between wildtypes and CNP mutants (Fig 16D). Therefore a loss of sensory fibers most likely affects all subpopulations. A prerequisite for normal amounts of myelinated fibers is a sufficient amount of myelinating Schwann cells. Therefore myelinating Schwann cell numbers were quantified. No alterations were detected in the amount of Schwann cell nuclei within saphenous nerves of 6 months old CNP deficient animals when compared to age matched wildtype controls (Fig 16E). 
A

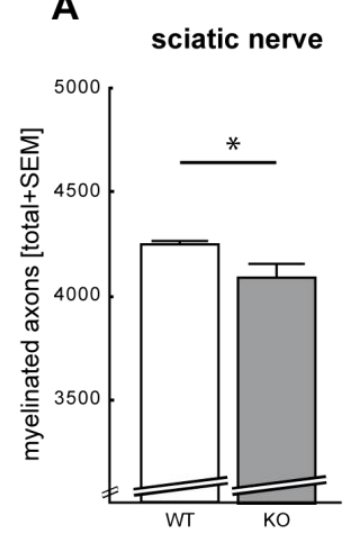

C saphenous nerve

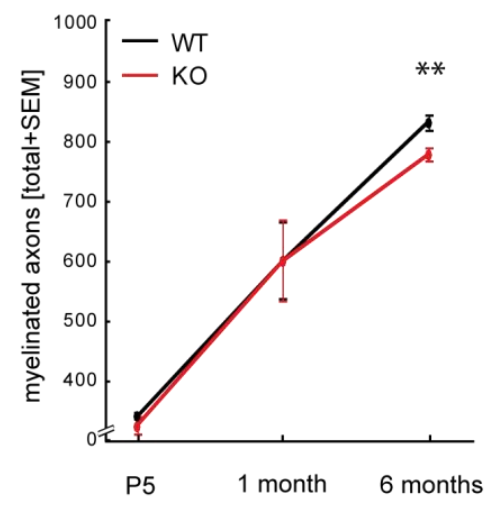

E saphenous nerve

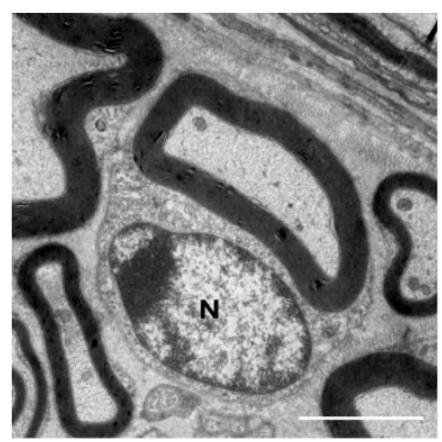

B
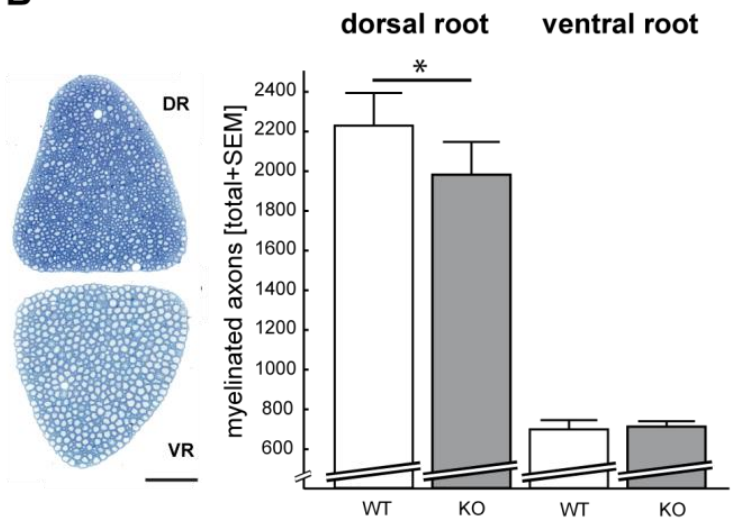

D

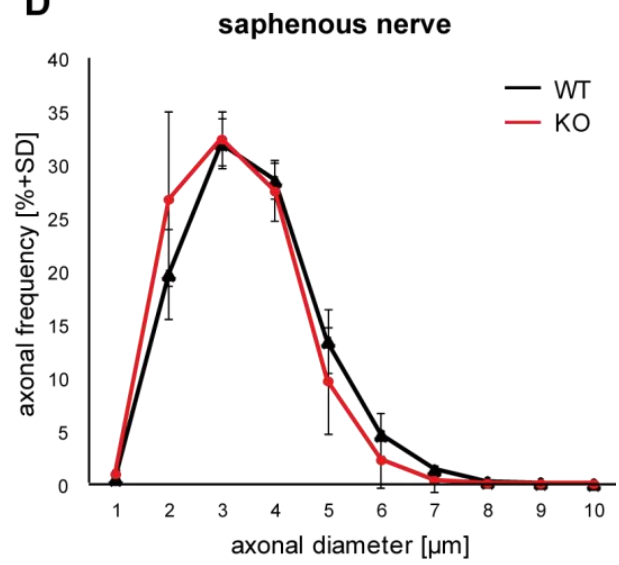

myelinating SC

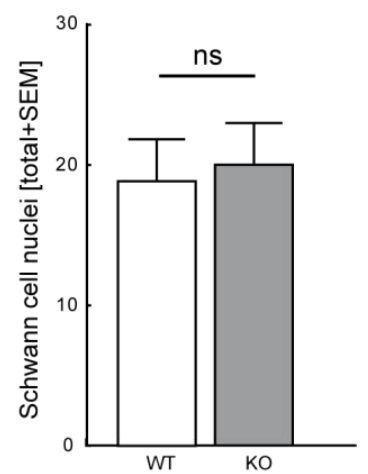

Fig 16: CNP deficiency causes adult onset sensory axonal loss

A: Quantification of myelinated fibers on sciatic nerve semithin cross sections of 6 months old wildtype (white) and CNP deficient animals (grey) reveals $4 \%$ loss of fibers. $(n=5$; Mean + SEM).

B: Representative image of a methylene blue / azur II blue stained semithin cross section of wildtype dorsal (DR) and ventral (VR) roots. Myelinated fiber numbers were quantified in wildtype (white) and CNP deficient animals (grey). Only dorsal roots display around $10 \%$ reduced myelinated fiber numbers, whereas no difference was detectable in ventral roots ( $n=7-8$; Mean + SEM; Scale bar of $100 \mu \mathrm{m})$.

C: Time course of myelinated fiber loss from P5 to 6 months old CNP deficient animals (red) compared to wildtype controls (black) in saphenous nerves shows a significant difference only in adult animals (7\%). (P5 $n=5$; $1 \mathrm{~m} n=4 ; 6 \mathrm{~m} \mathrm{n}=6$; Mean + SEM).

D: Axonal diameter measurements in saphenous nerves of 6 months old CNP-/- mice (red) and appropriate controls (black) show a normal frequency distribution also when CNP is deleted. (WT and KO $n=6$; Mean frequency of different axonal diameter groups and SD; column factor $=0.26$ ).

E: Representative electron micrograph of a myelinating Schwann cell and its nucleus $(\mathrm{N})$ in an adult saphenous nerve. Quantification of myelinating SC nuclei in 6 months old CNP-/- animals (grey) and age matched wildtype controls (white) show equal nuclei numbers. ( $n=6$; Mean + SEM; Scale bar of $2.5 \mu \mathrm{m}$ ). 
To further investigate if reduced myelinated fiber numbers in 6 months old CNP deficient animals actually reflect a loss of fibers or if the axons just fail to properly myelinate, but can be found as intact axons, refined investigations of unmyelinated axons larger than $1 \mu \mathrm{m}$ in diameter were performed. Big unmyelinated axons appear as nodal profiles (A), sorted big unmyelinated axons (B) and big axons in Remak bundles ( $\mathrm{C}$ in Fig 17). These profiles were observed as rarely in CNP deficient animals as in wildtype controls when quantified in whole saphenous nerves on electron microscopic level (Fig 17A-C). Total numbers were below $1 \%$. This suggests that the reduced myelinated fiber numbers indeed reflect a loss of fibers during adult life. In contrast to the CNS, axonal swellings were not observed more frequently in CNP deficient peripheral nerves (Fig 17D). This indicates a slow progressive but continuous loss of fibers.

A
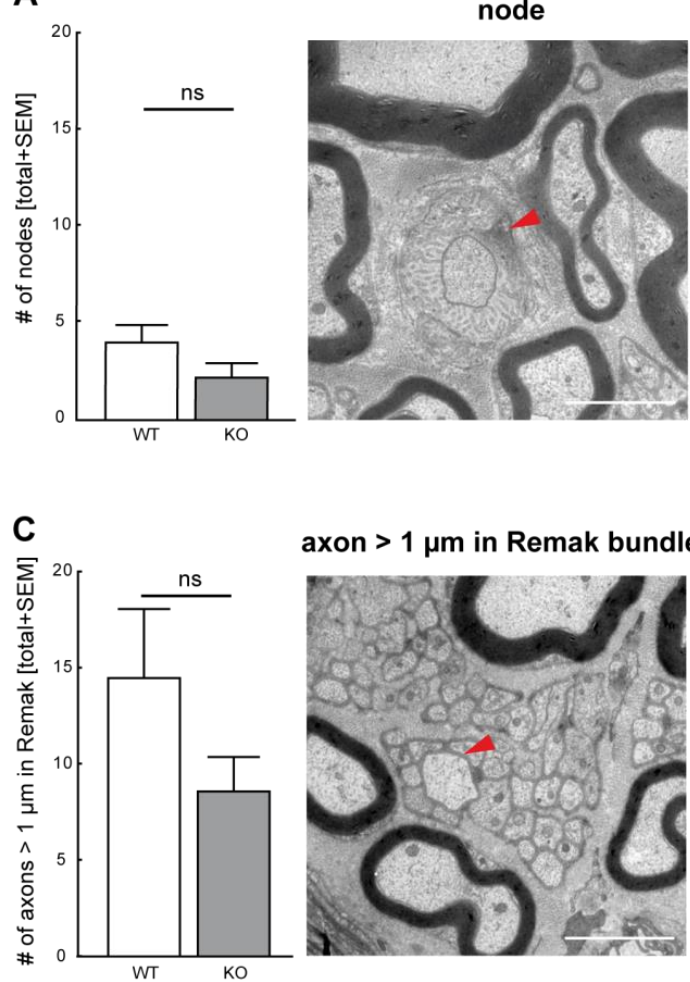

B

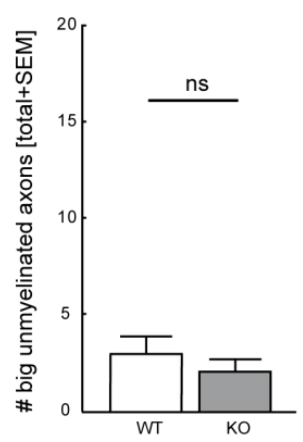

sorted big unmyelinate axon

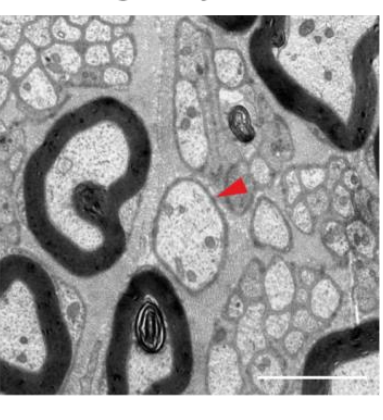

D
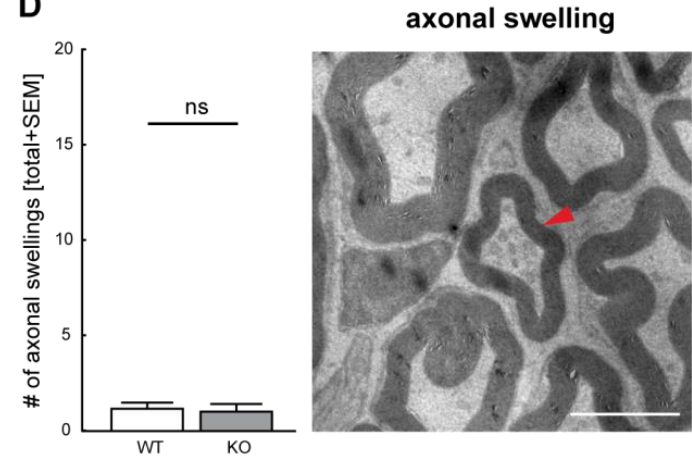

Fig 17: Unaltered frequency of big unmyelinated axons

A: Representative electron micrograph of a nodal cross section (arrowhead) in a conventionally fixed adult saphenous nerve. The quantification of total nodal numbers in 6 months old CNP deficient animals (grey) shows no difference to age matched controls (white). ( $n=6$ per group; Mean + SEM; Scale bar of $2.5 \mu \mathrm{m}$ ).

B: Representative electron micrograph of an unmyelinated axon above $1 \mu \mathrm{m}$ in diameter (arrowhead) in an adult saphenous nerve. Sorted but unmyelinated fibers come into view equally frequent in 6 months old CNP-/- animals (grey) and age matched wildtype controls ( $n=6$; Mean of total counts + SEM; Scale bar of $2.5 \mu \mathrm{m}$ ).

C: Representative electron micrograph of a Remak bundle containing an unsorted axon above $1 \mu \mathrm{m}$ in diameter (arrowhead) in an adult saphenous nerve cross section. No difference could be detected in total numbers of big unsorted axons in 6 months old CNP deficient animals (grey) and age matched wildtype controls (white) $(n=6$; Mean of total counts + SEM; Scale bar of $2.5 \mu \mathrm{m}$ ).

D: Representative electron micrograph of an axonal swelling (arrowhead) in an adult saphenous nerve. Total numbers of the rarely observable axonal swellings in 6 months old CNP-/- animals (grey) and age matched wildtypes (white) are equal. ( $\mathrm{n}=6$; Mean of total counts + SEM; Scale bar of $2.5 \mu \mathrm{m}$ ). 


\subsubsection{Remak bundles remain unaffected by CNP deficiency}

When speaking about sensory axonal loss or pathological alterations within SC of small caliber axons, it should be considered that the majority of sensory axons are unmyelinated C-fibers in Remak bundles that are associated with nonmyelinating SC. Even though adult nonmyelinating Schwann cells seem to express no or only very little CNP (see Fig 6) it should be excluded, that these fibers are affected, as their functionality is evenly crucial for peripheral nerve integrity. Refined analysis of CNP deficient saphenous nerves on electron microscopic level demonstrated that their Remak bundles are indistinguishable from those of wildtype controls at an age of 6 months. Bundle sizes (Fig 18B) as well as the quantities of axons that were improperly shielded from the endoneurium (Fig 18D) or their neighbouring axons (Fig 18E) were equal in mutants and controls. In line with that, the amount of nonmyelinating Schwann cells in CNP deficient animals showed wildtype levels as well (Fig 18C). An involvement of nonmyelinating SC in the pathological alterations in CNP deficient nerves can thus be considered as negligible.

A
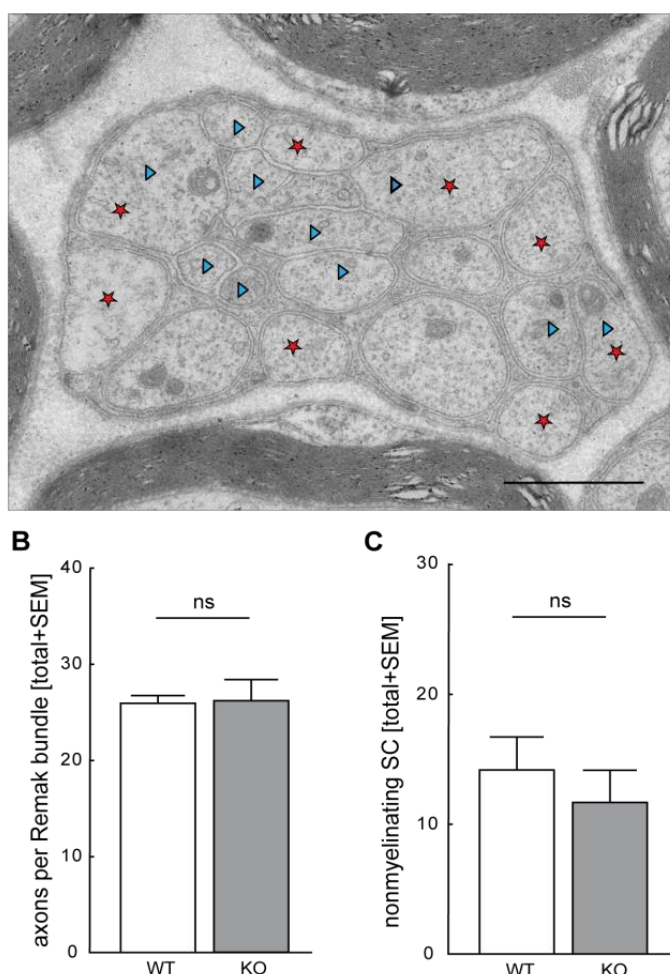

D

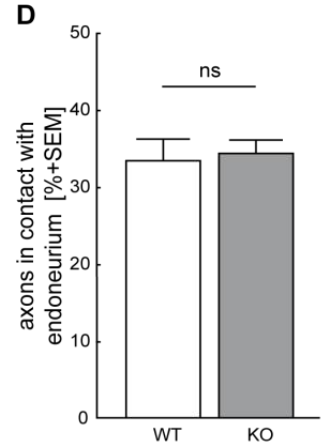

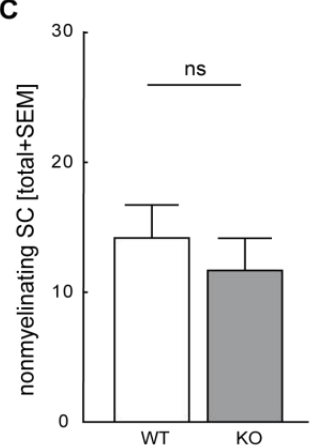

E

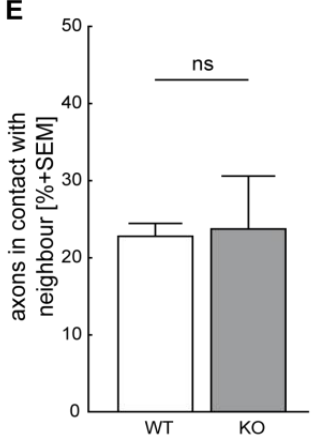

Fig 18: Remak bundles remain unaltered

A: Representative electron micrograph of a Remak bundle of a 6 months old wildtype saphenous nerve. Red stars highlight axons that are not completely insulate from the endoneurial space, whereas blue triangles depict axons that are incompletely separated from each other. (Scale bar represents $1 \mu \mathrm{m})$.

B: Quantifications of the total number of axons per Remak bundle show no differences in bundle size in 6 months old CNP deficient animals (grey) compared to wildtype controls (white). ( $n=3$; Mean + SEM).

C: The amount of nonmyelinating Schwann cell nuclei, quantified in saphenous cross sections of 6 months old CNP mutants (grey), is equal to that of age matched wildtype controls (white). $(n=6$; Mean + SEM).

D: Frequency quantification of axons that are not completely shielded from the endoneurium (red stars in A). CNP deficient mice (grey) and controls (white) display equal frequencies. $(n=3$; Mean + SEM).

E: Quantifications of axons that are in direct contact to their neighbours show similar frequencies in wildtypes (white) and CNP mutant mice (grey). ( $n=3$; Mean + SEM). 


\subsubsection{No indication of inflammation within peripheral nerves of CNP mutants}

In the central nervous system of CNP deficient mice neurodegeneration is accompanied by a severe neuroinflammation (Lappe-Siefke et al., 2003; Wieser et al., 2013). The PNS also displayed axonal degeneration which could potentially trigger an inflammation. An acute inflammation may on the other hand further enhance the axonal degeneration. However, immunohistological stainings for invading macrophages (MAC3) and a T-cell response (CD3) within peripheral nerves displayed equal amounts of positively labeled (brown) nuclei in 6 months old CNP mutant mice and wildtype controls. Both, the MAC3 (Fig 19A) and the CD3 staining (Fig 19B) exhibited low amounts of inflammatory cells in peripheral nerves. Contrary to the CNS, axonal loss in the peripheral nervous system was not accompanied or triggered by a neuroinflammation.

A
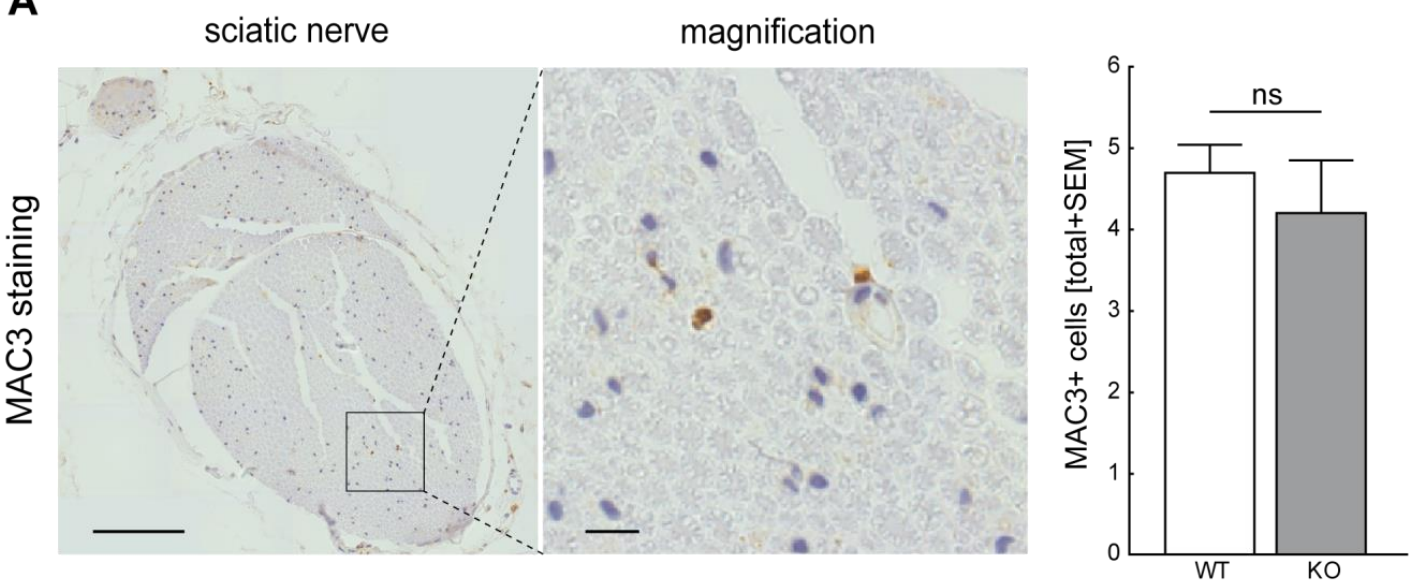

B


Fig 19: No indication of inflammation within the PNS of CNP deficient animals

A: Wax sections of sciatic nerves of 6 months old wildtype animals DAB stained for MAC3, a marker for macrophages. Left, overview image and on the right a magnification that illustrates the quantified MAC3 positive cells. Quantifications show equally low amounts of MAC3 positive cells in wildtype (white) and CNP deficient (grey) animals. (WT n=5, KO n=7; Mean + SEM; scale bars represent $100 \mu \mathrm{m}$ (left) and $10 \mu \mathrm{m}$ (right)).

B: Wax sections of sciatic nerves of 6 months old wildtype and CNP-/- animals stained with the T-cell marker CD3. On the left an overview image of a stained sciatic nerve and on the right a magnification of a CD3 positive cell. Quantifications show that CD3 positive cells are as rare in CNP mutants (grey) as in wildtype controls (white). (WT n=5, KO n=7; Mean + SEM; scale bars represent $100 \mu \mathrm{m}$ (left) and $10 \mu \mathrm{m}$ (right)). 


\subsubsection{CNP deficient animals display altered sensory nerve characteristics}

Myelin represents a high resistance electrical insulation for axons to increase conduction speed through saltatory impulse propagation. The myelin sheath geometry is believed to be optimized for maximal conduction speed (Donaldson and Hoke, 1905; Smith and Koles, 1970; Waxman, 1980). Hence alterations within the myelin sheath may influence electrical nerve characteristics. To this end, electrophysiological assessments on sciatic and respective tibial nerves were performed for adult CNP deficient and wildtype animals. Neither motor nerve conduction velocity (mNCV) nor the evoked compound muscle action potentials (CAMPs) within the small plantar foot muscles were altered in adult CNP deficient animals (Fig 20A-C). In contrast, sensory nerve potentials tend to be decreased and the sensory NCV was shown to be slightly slower in peripheral nerves of adult CNP deficient animals compared to age-matched controls (Fig 20D-F). Most probably the slowing in sensory conduction velocity was due to the altered myelination of small caliber axons. The reduced amount of sensory axons may have an impact on electrical characteristics of the nerve and may explain the trend to a decrease in sensory potentials.

\subsubsection{CNP mutants display impaired sensation}

All aforementioned findings implicate alterations in sensory nerve fibers. If these alterations lead to a general impairment within the PNS functionality and influence the sensation of CNP-/- mice was so far unknown. To this end we examined motor and sensory functionality using independent behavioural tests. The grid slip and bar test involve higher motor coordination, whereas the grip strength test resembles grasping reflex behaviour paired with muscle power. Within the grid slip and bar tests (Fig 21A-B) 8 months old CNP deficient animals displayed apparent difficulties reflected in more slips and dramatically shorter holding times on the bar. As those two tests depend on higher motor coordination, an effect of the CNS, which is severely affected at that age, cannot be excluded. In contrast, CNP deficient animals had no difficulties within the grip strength test (reflex arch with minimal CNS involvement) which was in line with the unaltered large motor fibers (Fig 21C). On the other hand, the mechanical and thermal plantar tests may also resemble reflex related behaviour (avoidance reflex) but here the sensory component was addressed. Both tests showed significantly longer latency for paw withdrawal (Fig 21D-F) in CNP deficient animals compared to wildtype controls. The hyposensitivity seems to be the result of the disturbed integrity of sensory (small) peripheral nerve fibers. Therefore we conclude that CNP deficiency not only causes alterations within the SC, but secondarily impairs axonal integrity and subsequently affects the whole organism. 
A

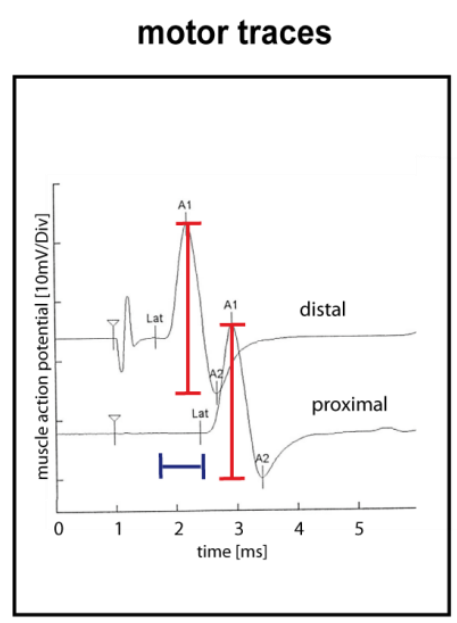

D

sensory trace

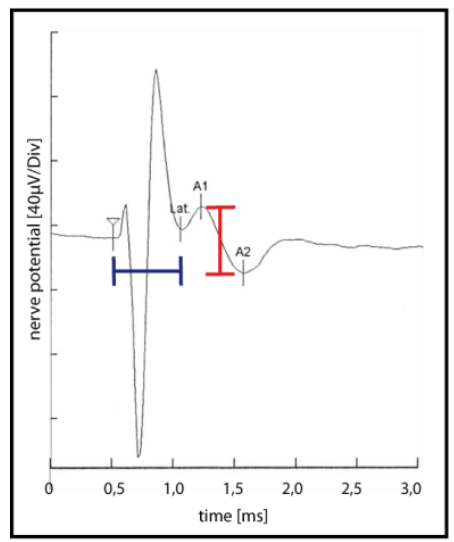

B

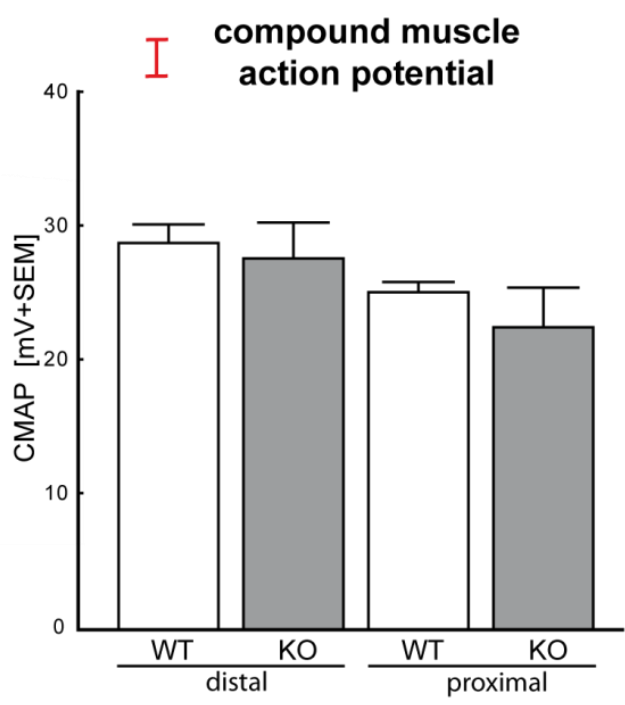

E
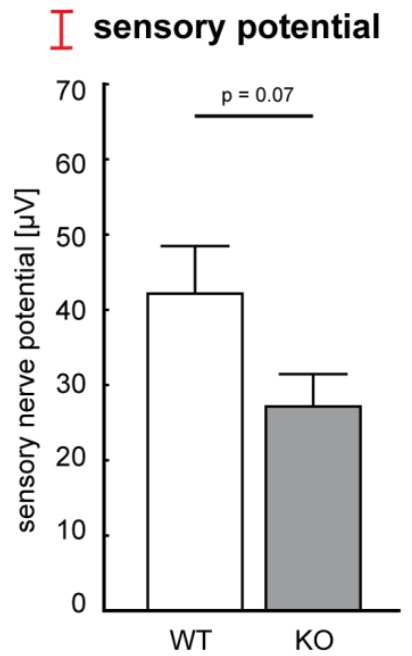

C

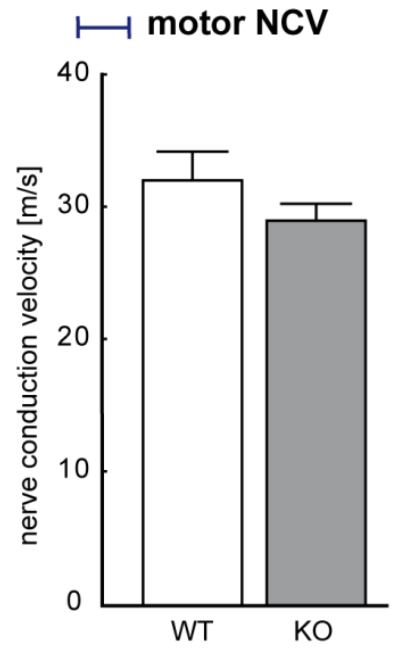

F

$\longmapsto$ sensory NCV

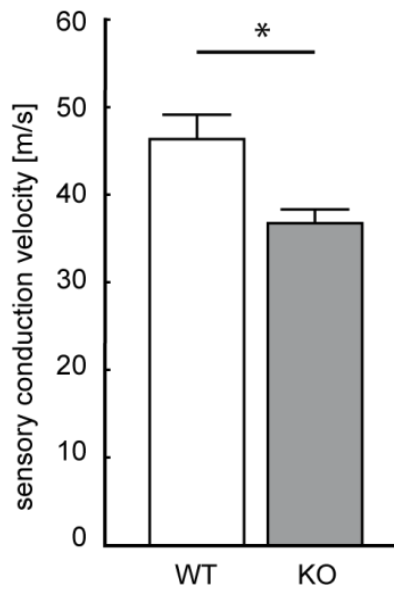

Fig 20: Altered electrophysiological characteristics of CNP deficient peripheral nerves

A: Trace of intramuscular recordings after distal and proximal sciatic / tibial nerve stimulation. Amplitudes are highlighted in red and the latency difference between the two recordings (blue) allowed the calculation of the nerve conduction velocity (latency / distance of electrodes). (time scale $1 \mathrm{~ms}$; amplitude scale $10 \mathrm{mV} /$ Division).

B: Electrophysiological assessment of wildtype (white) and CNP deficient animals (grey) at 8 months of age display no significant changes of amplitudes after distal or proximal stimulation. ( $n=6$; Mean $+S E M)$.

C: Motor nerve conduction velocities are indifferent between CNP deficient (grey) animals and controls (white) with 8 months of age. $(n=6$; Mean + SEM).

D: Example of a sensory trace (peaks are the stimulation artifacts). Difference of maximum and minimum of the recording resembles the amplitude of the sensory nerve potential (red). The sensory nerve conduction velocity is calculated from the latency of the signal (blue) and the distance of the electrodes (time scale 0.5 ms; amplitude scale $40 \mu \mathrm{V}$ / Division).

E: The quantification of the sensory nerve potential after distal stimulation, shows a trend towards reduced nerve potentials in 8 months old CNP deficient animals (grey) compared to controls (white) but measurements are very variable. (WT $n=7$, CNP-/- $n=6$; Mean + SEM).

F: The sensory conduction velocity, calculated from the latency of response (blue) and the distance of the electrodes, displays $21 \%$ slower conductance in CNP deficient animals (grey) at 8 months of age. (WT n=7, CNP/- $\mathrm{n}=6$; Mean + SEM). 
A
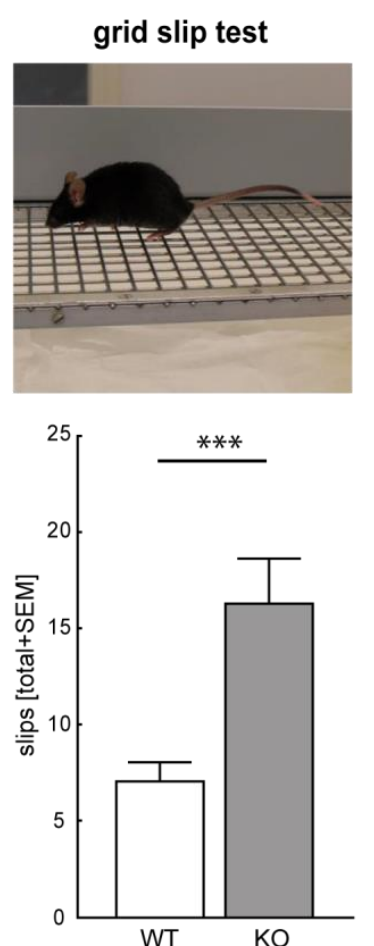

D

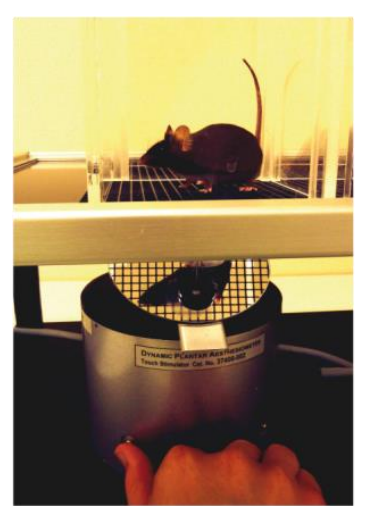

B
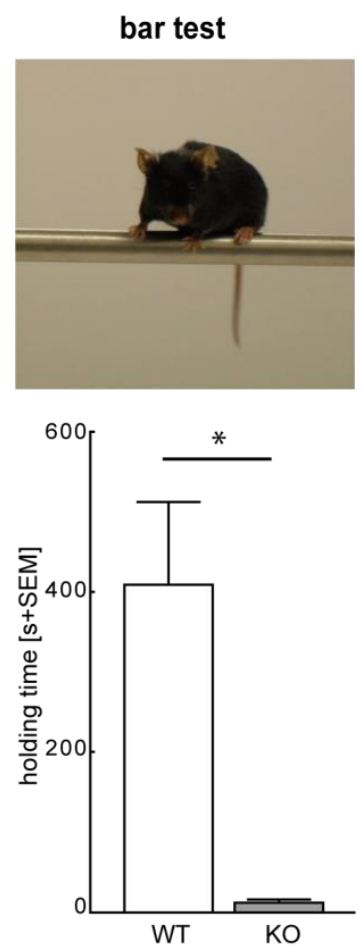

E

mechanical plantar

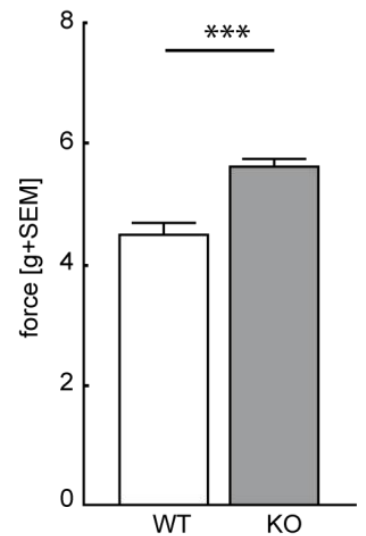

C

grip strength test
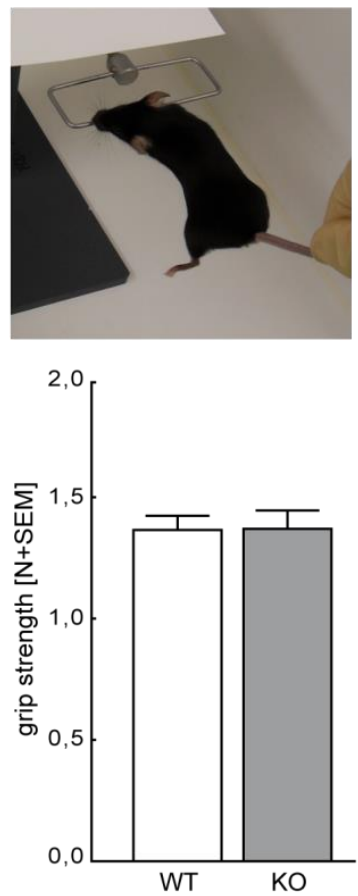

$\mathbf{F}$

infrared plantar

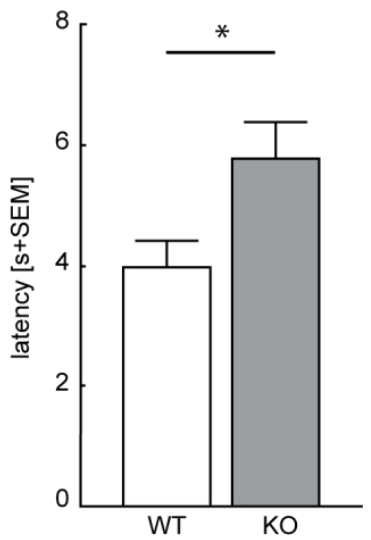

Fig 21: Impaired sensation and higher order motor coordination

A: Mouse performing on a grid (grid size $1 \times 1 \mathrm{~cm}$ ). Quantification of slips along the $2 \mathrm{~m}$ grid show more than double the amount of slips for 8 months old CNP deficient animals (grey) compared to wildtype controls (white). (WT $n=7, K O n=5$; Mean + SEM).

B: Experimental animal performing on a bar. CNP deficient mice (grey) with 8 months of age are hardly able to stay on the bar compared to controls (white) at the same age. (WT $n=7, K O n=5$; Mean + SEM).

C: Mouse grabbing the horizontal bar and investigator pulling it away to perform the grip strength test. Quantifications of this grasping reflex behaviour (together with the actual muscle power) show no alteration in CNP deficient animals (grey) compared to controls at 8 months of age (white). (WT $n=7, K O n=5$; Mean + SEM).

D: Mouse in the plantar aesthesiometer setup prepared for mechanical plantar testing.

E: Quantification of the mechanical force that is needed to provoke foot withdrawal reveals that 8 months old CNP deficient animals (5.7x g-force; grey) respond only to higher forces compared to age matched wildtype controls (3.9x g-force; white). (WT $n=14, K O n=21$; Mean + SEM).

F: Quantification of withdrawal latencies after constant thermal stimulation of the plantar surface display $2 \mathrm{~s}$ longer irradiation times to initiate paw withdrawal in 8 months old CNP deficient mice (grey) compared to the control group (white). (WT $n=14, K O n=16$; Mean + SEM). 
In summary, it was shown that Schwann cells display different molecular compositions of their myelin sheaths according to the size of the underlying axons. Remarkable differences were observed for the CNP protein abundance, which was elevated in SC associated with small caliber axons. Ablation of CNP induced a reduction of the noncompact myelin compartment and drives hypermyelination in adult animals. These alterations were particularly pronounced in Schwann cells that normally express most CNP. Consequently, these SC displayed the most prominent imbalance of the two compartments. This likely deteriorated intracellular and transcellular transport processes which potentially have impaired the axonal support. Subsequently, this may have caused the observed axonal loss of small caliber axons, which possibly led to the sensory impairment. 


\section{Discussion}

The peripheral nervous system (PNS), which connects the central nervous system (CNS) with all peripheral organs, consists of heterogeneous peripheral axons and their associated Schwann cells (SC). Peripheral nerve fibers are usually classified with respect to their direction of signal transduction, diameter, existence of a myelin sheath and conduction speed (FitzGerald et al., 2011). Signaling direction and diameter are neuron intrinsic characteristics whereas the formation of a myelin sheath and the conduction speed are mainly defined by the associated Schwann cells. Small fibers below $1 \mu \mathrm{m}$ in diameter together with nonmyelinating SC are referred to as Remak bundles. All remaining fibers ranging from small sensory fibers barely above $1 \mu \mathrm{m}$ in diameter up to large motor axons are enwrapped by myelinating SC that form the compact myelin sheath. Differences within the population of myelinating SC have previously been reported in peripheral nerve regeneration studies that revealed a preferential reinnervation. These observations were attributed to the different molecular architectures of the extracellular matrices and the individual expression of trophic factors by denervated motor and sensory SC (Höke et al., 2006; Madison et al., 2009; Brushart et al., 2013). It was not yet investigated if motor and sensory SC express differences with regard to their myelin sheaths in uninjured nerves. Therefore, this work aimed to identify developmentally established differences in the myelin sheath composition between myelinating SC subpopulations. The second and major part of this work addresses the impact of the differentially expressed noncompact myelin protein CNP on distinct peripheral nerve fiber types. CNP is involved in axonal support independent of myelination within the CNS and has been shown to influence the process of compaction during CNS development (Lappe-Siefke et al., 2003; Snaidero et al., 2014). To uncover the functional relevance of CNP within Schwann cells, peripheral nerves of CNP deficient mice were investigated with special emphasis to distinct fiber types.

\subsection{Schwann cells are diverse}

In vitro- as well as regeneration studies on motor and sensory nerves indicated that Schwann cells express discrete "motor" and "sensory" phenotypes specifically promoting the outgrowth and regrowth of appropriate motor or sensory axons during development and peripheral nerve regeneration. This capacity was related to the differential expression of trophic factors and extracellular matrix proteins (Brushart, 1993; Höke et al., 2006; Hu et al., 2010). The phenotypic memory of dedifferentiated SC (Büngner repair cells) will only be lost during prolonged denervation or by artificial expansion of the SC in vitro. Apart from trophic factors, several myelination and signaling related genes displayed differences in rat motor and 
sensory nerve RNA profiling studies (Jesuraj et al., 2012). This indicated a more general diversity of SC beyond the expression of trophic factors. One crucial task of a SC is the formation of the myelin sheath which needs to be tightly regulated according to the specific axon. Therefore we hypothesized, that myelinating SC are not uniform but that their myelin constitution is precisely adjusted to axonal demands. Hence, the first aim of this work was the investigation of individual myelin characteristics of different fiber types as they have been described for the just mentioned trophic factors and extracellular matrix proteins. To this end, myelin that was purified from SC of mainly large caliber motor axons (ventral roots) was compared to dorsal root myelin (sensory predominantly small caliber axons). Numerous differentially regulated proteins were observed in silver impregnations (Fig 2A) of purified myelin. This was confirmed by comparative mass spectrometry based dorsal and ventral myelin proteome analyses and additional immunoblot investigations for classic compact and noncompact myelin proteins. Apart from classical myelin proteins also several proteins related to metabolism or cytoskeleton, that just recently have been linked to myelin (Jahn et al., 2009; Patzig et al., 2011; Monasterio-Schrader et al., 2012), displayed differential expression levels (Fig 2B). Among the unequally expressed proteins, CNP revealed the most robust difference in protein abundance. These data suggest that SC are not only different with regard to the constitution of their extracellular matrices and the expression of trophic factors but also show a striking variability in their myelin sheath composition. In contrast to the reported differences in peripheral nerve regeneration, the newly observed variability in the myelin stoichiometry must have been established during SC development and is maintained throughout life. We speculate that the myelin specific differences are at least partially regained during SC redifferentiation and remyelination even though the myelin sheath never reaches the original sheath thickness in regenerating peripheral nerve fibers (Schröder, 1972). Altogether, this points to a general diversity of myelinating SC, which may enable them to individually match axonal demands. Furthermore, differences in dimensions of the myelin sheaths of small and large caliber axons may require an optimized relation of the compartments to enable efficient transport within the SC and among the SC-axoninterface. Thus, we believe that myelinating glia and their underlying axon are much more synchronized than so far assumed. 


\subsection{CNP is enriched in SC surrounding small caliber axons}

Among the investigated myelin proteins the most robust discrepancy in motor and sensory expression was observed for the noncompact myelin protein CNP. Quantitative immunoblot examinations revealed 2.7 fold higher protein abundance in dorsal root myelin compared to myelin harvested from ventral roots (Fig 3). This dissimilarity was confirmed by immunohistological assessments of dorsal and ventral roots, which similarly showed stronger expression of CNP in myelinating SC surrounding small caliber axons (Fig 4). Thus, enhanced signal intensities can be found in dorsal roots because of the proportion of small caliber axons which is much higher in dorsal compared to ventral roots. However, also small caliber fibers in the ventral roots display enhanced CNP expression levels. Hence, it can be concluded that the total CNP expression levels inversely correlates to the fiber diameter independent of the motor or sensory identity of the engulfed axon. As proposed for the CNS, CNP may be involved in axonal support independent of the myelin sheath also in the PNS (Lappe-Siefke et al., 2003; Nave, 2010a). In the CNS small caliber axons have repeatedly been reported to be more effected by pathologic alterations within the myelinating glia (Griffiths et al., 1998; Evangelou et al., 2001; Edgar et al., 2004, 2009). Thus, small caliber axons seem to be more vulnerable to subtle changes within the axon-glia interplay. One possible reason may be that they have higher demands for metabolic and trophic support as they seem to have the lowest storage capacities. This implies that also SC surrounding small caliber fibers may have to support their axons more intensely. CNP is proposed to be directly involved in axonal support and therefore elevated CNP levels may resemble SC's adequate response to enhanced trophic demands by small caliber axons. Interestingly, we could not detect CNP in nonmyelinating SC that engulf C-fibers in Remak bundles (Fig 6). This suggests that mature nonmyelinating SC do not express CNP, or that the level of expression is very low. However, during early development, most probably in the immature SC stage, also cells that gave rise to nonmyelinating SC must have expressed CNP as indirectly shown by CNP-Cre-driven molecular alterations that also affect Remak bundles. A good example is the CNP-Cre mediated ablation of PTEN which causes aberrant SC wraps around C-fibers, indicating that nonmyelinating SC were indeed reprogrammed using the CNP-specific promotor activities to drive Cre recombination (Goebbels et al., 2010). If CNP acts as a spacer to keep myelin sheaths uncompacted as seen in development (Snaidero et al., 2014) and upon CNP overexpression (Gravel et al., 1996), this function may have been abandoned during maturation of nonmyelinating SC as there is no compact sheath in nonmyelinating SC. Nevertheless, CNP is one of the earliest markers within the SC lineage (Scherer et al., 1994) and additional functions of CNP, probably the catalytic function, may be crucial in early development of nonmyelinating as well as myelinating SC. 


\subsection{CNP affects the noncompact and compact myelin compartment}

2',3'-cyclic nucleotide-3'-phosphodiesterase (CNP) is not only expressed in SC but also in oligodendrocytes within the CNS. Former investigations of CNP in the nervous system revealed that it is localized to all uncompacted myelin domains: paranodal loops, inner and outer mesaxons, the perinuclear cytoplasm and in the PNS also to Schmidt-Lanterman incisures. Therefore CNP belongs to the group of classic noncompact myelin proteins (Braun et al., 1988; Trapp et al., 1988). Within the developing CNS, CNP seems to carry out mainly structural functions in organization and maintenance of noncompact myelin regions but also influences the compact myelin sheath. Thus CNP overexpression severely impairs compaction due to an overload of CNP within the sheath which simultaneously hampers MBP pathfinding and thus the compaction of the myelin lamellae. Additionally, CNP overexpression induces aberrant myelin production (Gravel et al., 1996; Yin et al., 1997). This suggests CNP as a spacer within growing myelin, which normally gets coordinatedly subtracted and thus allows zipping of the myelin layers by MBP. Moreover, recent investigation by Snaidero and coworkers (2014) supported this hypothesis as the compaction speed was significantly increased in CNP deficient mice. Additional proof for an important role of CNP for the noncompact myelin integrity was given by the frequent observation of swollen inner tongues and disorganized paranodes in CNP deficient animals in the CNS (Rasband et al., 2005; Edgar et al., 2009). Together, these findings imply a critical involvement of CNP in noncompact myelin organization. Interestingly, even though CNP deficient mice develop a severe white matter atrophy and astrogliosis, the compact myelin sheath within the CNS appeared normal. This led to the assumption that CNP plays an important role in myelin independent axonal support (Lappe-Siefke et al., 2003; Nave and Trapp, 2008; Nave, 2010a, 2010b). The role of CNP in SC and its importance for PNS integrity has not been previously investigated. The striking differences in CNP protein abundance in different fiber types found in this study helped to uncover the impact of CNP function for different peripheral nerve fiber types. Initial investigations addressed the noncompact myelin, the compartment that normally comprises CNP. In line with reported increased compaction speed and a decrease in cytoplasmic channel frequencies (Snaidero et al., 2014) also SC display a reduction in noncompact myelin regions as inner mesaxon, cytoplasmic channels and inner tongues (Fig 7, 9, 10). Contrary to findings in the CNS, these observations were made in adult PNS tissue, whereas young CNP deficient peripheral nerves were largely unaltered. This pointed to a greater role of CNP for maintenance of SC integrity than for its impact on SC development. All findings point to a constricted noncompact myelin network whose functionality is pivotal for innermyelinic transport processes. Inner tongues, the actual axon-glia interface, were found to be smaller in the PNS of CNP mutants (Fig 8), which may directly hinder transcellular support processes. Swollen 
inner tongues as found in the CNS (Edgar et al., 2009) have almost never been seen in peripheral nerves of both genotypes. Swollen inner tongues potentially develop when the whole transport system within the glial cell collapses and no recirculation can take place causing an accumulation of material in distal glial regions (inner tongue). Theoretically this could happen in SC as well if peripheral nerves would reach the same level of degeneration as observed in the CNS. Contrary to the CNS, the ablation of CNP moreover has an impact on the size of the compact myelin compartment within peripheral nerves. CNP deficiency causes an adult onset hypermyelination. The myelin sheath thickness was normal during development and just slightly increased in juvenile stages, whereas additional ultrastructurally normal compact myelin wraps are accumulated during adulthood. Interestingly, the hypermyelination is more pronounced for small caliber axons. Hence, the magnitude of hypermyelination is indirectly proportional to axonal diameters and independent on motor and sensory characteristics of the axon (Fig 11). These data suggest that CNP is involved in adult negative regulation of myelination. As proposed by Snaidero and coworkers (2014), also in the PNS CNP may act as a physical spacer between neighbouring myelin layers and consequently when the spacer is ablated noncompact areas are further zipped and therefore reduced. Especially in the inner tongue ongoing addition of newly synthesized myelin components may not be blocked when the CNP-brake is released and thus allows for ongoing myelination. Together, the findings, that SC of small caliber axons express the most CNP and that they show the strongest hypermyelination when CNP is ablated led to the assumption, that the more CNP a SC expresses the more it relies on its myelin regulatory function. Contrary to other hypermyelinating mutants, CNP deficiency did not enhance the formation of tomacula or myelin inclusions, outfoldings or pathological alterations of Remak bundles (Fig 12) as described for the ablation of PMP22, PTEN or DLG1 (Adlkofer et al., 1995; Goebbels et al., 2010, 2012; Cotter et al., 2010). This suggests different underlying mechanisms that all provoke excess myelin growth with distinct characteristic phenotypes. 


\subsection{Molecular alterations upon CNP deficiency}

Even though CNP is one of the earliest markers within the SC lineage, its relative mRNA expression profile resembles that of other myelin genes like PLP and MBP (Scherer et al., 1994). Interestingly, mRNA profiling studies with CNP overexpressing mutants revealed an earlier peak in myelin gene expression patterns (Gravel et al., 1996; Yin et al., 1997). Therefore we hypothesized, that the ablation of CNP may also interfere with regulatory mechanisms on transcriptional level and thus may shift the peak of myelin gene expression out of the critical window, possibly prolonging the myelination up to adult stages. Contrary to our expectations, quantitative mRNA analyses provided no evidence of altered myelin gene specific transcript levels for PLP, P0, MBP or MAG at any tested time point (Fig 13). Therefore we concluded that the deficiency of CNP does not impair myelination control on transcriptional level in peripheral nerves. This further imposed, that the adult onset hypermyelination of small caliber axons likely arose by changes in translational control or by altered lipid and myelin protein stability. Myelination is generally promoted by active PI3K/AKT and MAPK/ERK signaling during development (Taveggia et al., 2005; Maurel and Salzer, 2000; Pereira et al., 2012; Newbern et al., 2011; Ishii et al., 2013; Sheean et al., 2014). Activity states of the pathways generally correspond to the level of phosphorylated (active) signaling molecules. In order to finish myelination, active promyelinating pathways have to be down regulated. This is mediated by a decrease of PI3K/AKT and MAPK/ERK pathway members and especially a down regulation of the phosphorylated isoforms. Additionally the activity of the translational machinery will be decreased (Sheean et al., 2014). This finally slows myelin protein and lipid production to reach a steady state level to maintain the established myelin sheath throughout life. Both above mentioned pathways can influence myelination in either direction. Thus, negatively regulated PI3K/AKT signaling as described for conditional ablation of PTEN, DLG1 or DDIT4/REDD1/RTP801 induces hypermyelination (Noseda et al., 2013; Goebbels et al., 2010; Cotter et al., 2010; Macklin, 2010). In contrast, constitutive activation of PI3K/AKT signaling has either no effect on peripheral myelination (PLP-AKT-DD, Flores et al., 2008), or even causes hypomyelination (EGR2-PI3K-DA, Sheean et al., 2014). On the other hand, hypermyelination of peripheral axons can be provoked by constitutive activation of ERK signaling in SC (from precursor stage on), which drives continued myelin growth (Ishii et al., 2013; Sheean et al., 2014). In contrast, acute activation of ERK signaling post maturation (P0-RafTR mutants or in vitro) leads to peripheral nerve demyelination (Napoli et al., 2012; Harrisingh et al., 2004). Therefore, only a coordinated regulation of both pathways ensures a matched myelin sheath synthesis for each axon. Interestingly, adult dorsal root preparations of CNP deficient animals displayed an increased activity for both pathways (Fig 14) compared to wildtype dorsal roots. The balanced upregulation of MAPK/ERK and PI3K/AKT signaling may abolish 
demyelinating effects as expected for pure increased AKT signaling or reactivated ERK signaling. Therefore, these alterations more likely resemble ongoing myelination as found in development. This suggests, that CNP is involved in the negative regulation of myelination when SC mature. Even though no general upregulation of the translational machinery, as described for MEK1DD mutants (Sheean et al., 2014) has been found in CNP deficient peripheral nerves, some myelin proteins (MBP and MyP2) and lipid related genes (HmgCR; SCAP) are elevated upon CNP deficiency (Fig 15). These findings additionally point to an ongoing myelination but moreover indicate an altered myelin stoichiometry in CNP deficient nerves. How CNP function influences the activity of the PI3K/AKT and MAPK/ERK signaling pathways can only be speculated. Schwann cells are equipped with $A 2_{a}$ adenosine receptors and the PNS may also express the extracellular adenosine pathway as described for the brain (Stevens et al., 2004; Stone et al., 2009; Verrier et al., 2012, 2013). Here, CNP as catalytic enzyme may come into play. It can hydrolyze and therefore detoxify 2',3'-cyclic nucleotides, that are found during RNA breakdown. Generated 2'-phosphonucleotides will further be degraded to their respective nucleotides. Here, for example adenosine can on its own promote axonal support and neuronal survival (Thompson et al., 1994; Jackson et al., 2009). Hence, deletion of CNP would cause accumulation of 2',3'-cyclic nucleotides and theoretically increased levels of their appropriate 3',5'-cyclic isomers. Elevated 3',5'-cyclic AMP (cAMP) level have been shown to promote Schwann cell differentiation and myelin gene expression via ERK1/2 activation (Arthur-Farraj et al., 2011; Stevens et al., 2004). This provides a possible link of the catalytic function of CNP and the observed elevated signaling pathways in adult CNP deficient peripheral nerves that potentially drives hypermyelination.

\subsection{CNP deficiency impairs small fiber integrity}

Up to the present, all discussed observations only referred to the integrity of SC. The observation, that CNP deficiency causes a severe neurodegeneration without a myelination defect (in the CNS) led to the assumption, that glial cells have supportive functions independent of myelination and that CNP may be directly involved in the axonal support (Lappe-Siefke et al., 2003; Nave and Trapp, 2008; Edgar and Nave, 2009; Nave, 2010a). CNP may have a similar direct supportive function within the PNS. Investigations of the axonal integrity in peripheral nerves revealed an adult onset loss of myelinated fibers, which is pronounced in sensory nerves (Fig 16,17). However, a loss of myelinating and nonmyelinating SC has not been observed. A direct comparison to the CNS, which displays severe white matter atrophy upon CNP deficiency, reveals that the magnitude of degeneration is markedly lower within the PNS (Lappe-Siefke et al., 2003; Edgar et al., 2009). The discrepancy might at least partially be explained by the ten times lower 
expression level of CNP in the PNS compared to the CNS (Kurihara and Tsukada, 1967). However, the predominance of fiber loss to small caliber axons has been reported for small caliber central nervous system fibers as well (Edgar et al., 2009) and therefore suggests a common function of CNP in axonal support for small caliber central and peripheral nerve fibers. How exactly CNP performs its supportive function is not yet clear. CNP is supposed to be involved in the structural maintenance of the noncompact myelin network by stabilizing membrane-cytoskeleton interactions (Bifulco et al., 2002; Lee et al., 2005). Ablating the structural functionality of CNP caused the bidirectional impairment of the compartments, with a reduction of the noncompact transport routes and an additional increase of the compact myelin which further elongated transport routes (Fig 8-11). A deterioration of the axonal support due to defective SC intrinsic and transcellular transport through the constricted axonSC interface can only be speculated. Therefore, we cannot exclusively answer the question if axonal loss is only caused by a trophic or metabolic undersupply due to the lack of CNP's structural function or if the perspective function might be more direct as in the CNS, where imbalance of the compartments has not been observed. However, the pronounced sensory axonal loss and hypermyelination can further be correlated with an impaired peripheral nerve functionality, which is reflected in a hyposensitivity for mechanical and thermal stimulations in adult CNP deficient animals (Fig 19-21). Taken together, Schwann cells of small caliber axons that would normally express most CNP seem to rely most on its function. Hence, the ablation of CNP primarily impairs sensory SC integrity, which secondarily leads to a loss of small caliber peripheral axons, deteriorating peripheral nerve integrity and functionality. Thus the noncompact myelin protein CNP is of particular importance for the integrity of small peripheral nerve fibers. 


\subsection{Model of CNP deficiency}

Within this work it has been shown, that SC exhibit different molecular compositions of their myelin sheaths according to the caliber of the engulfed axon. This led to the notion that myelinating SC are diverse and that they may individually adapt to the underlying axon. Here, CNP was found as one of the most differentially expressed proteins between SC types. Thus, SC of small caliber axons express most CNP and may therefore rely most on its function, making them more vulnerable to pathological alterations when CNP is ablated. The deficiency of CNP causes a remarkable hypermyelination of small caliber axons, which suggests an involvement of CNP in negative regulation of adult peripheral myelination likely through suppression of active signaling pathways. Moreover, loss of CNP as spacer causes a reduction of the noncompact myelin compartment which may by itself facilitate ongoing growth of the compact myelin sheath in small caliber axons. The imbalance of the compartments and the altered myelin stoichiometry likely deteriorate innermyelinic and transcellular transport processes. Alterations within SC may thus secondarily impair axonal support especially for small fibers which are finally lost. In contrast to normal appearing CNS myelin, ablation of CNP provokes a specific adult onset PNS phenotype that includes alterations within the myelin sheath and impaired axonal integrity. We propose that CNP serves discrete but partially overlapping functions in SC and oligodendrocytes. In summary, SC exhibit diverse molecular architectures of their myelin sheaths and the differentially expressed protein CNP is particularly important for the integrity of small myelinated fibers in the adult PNS.

Wildtype

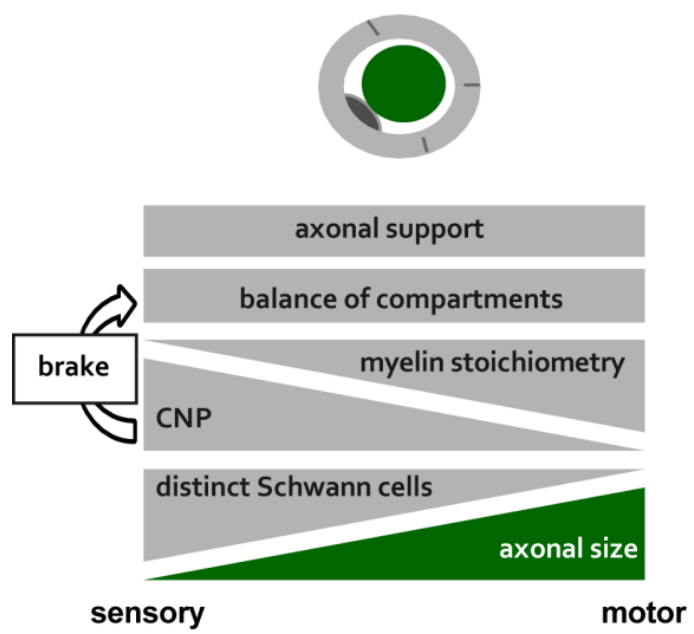

CNP-deficiency
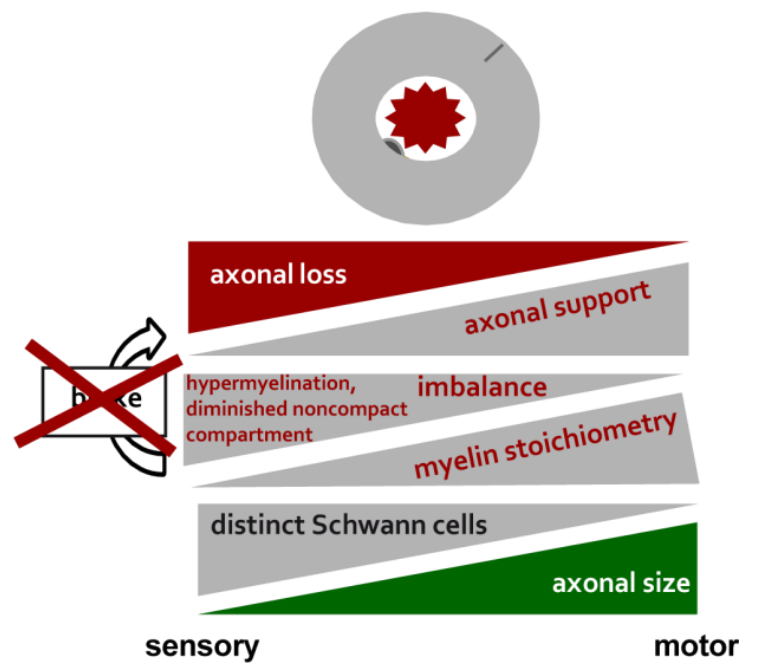

Fig 22: Model of SC diversity and the differential impact of CNP on distinct PNS fibers

The heterogeneous peripheral axons are associated with molecularly diverse SC. Different SC are equipped with a discrete myelin stoichiometry with CNP being enriched in SC surrounding small caliber axons. CNP acts as a brake for ongoing adult myelination and is thus involved in myelin sheath homeostasis. Moreover, SC exhibit trophic and metabolic support functions that likely depend on the integrity of the noncompact myelin compartment. When CNP is ablated, the myelin stoichiometry and the balance of compact and noncompact myelin are altered, especially in SC around small caliber axons. Therefore axonal support may be hampered leading to loss of small caliber axons during adulthood, which finally impairs sensation. 


\subsection{Future directions}

In this work it has been shown that the composition of myelin sheaths surrounding small compared to large caliber axons differs. This led to the suggestion that myelinating SC are diverse. We hypothesize, that differences in myelin constitution resemble adaptations of SC to meet their own demands regarding the wide range of sheath dimensions, and to cope with individual requirements of discrete axonal subpopulation. In the past few years the number of known myelin proteins tremendously increased as mass spectrometry based myelin proteome analyses identified a broad spectrum of know but also unknown proteins as constituents of the myelin sheath (Jahn et al., 2009; Patzig et al., 2011; Monasterio-Schrader et al., 2012). In light of this, we initiated a second more in depth proteome analysis with more sensitive instrumentation to compare the molecular constitution of dorsal and ventral root myelin. This will allow for detection of less abundant differentially regulated myelin proteins and may moreover lead to the identification of myelin protein clusters that are essential for specific peripheral fiber types. Subsequent analysis of distinct fiber types in mouse models of further differentially expressed proteins will raise our understanding of specific tasks of myelin proteins for discrete fiber types.

Furthermore these data are useful to better understand pathomechanisms of hereditary peripheral neuropathies that specifically affect motor or sensory fibers. For instance in Charcot-Marie-Tooth disease type 1A, the most common peripheral neuropathy (CMT1A PMP22 duplication), pathological alterations differ with respect to the fiber type with progressive demyelination in large and hypermyelination of small fibers (Fledrich et al., 2014). Upcoming analyses of CNP levels in the CMT rat model will evaluate an involvement of CNP in the general disease etiology.

In relation to the specific PNS phenotype of CNP deficient nerves it would be highly interesting to analyze the direct consequences of imbalanced myelin compartments. We propose that the increase of compact and decrease of noncompact myelin hampers the innermyelinic transport and finally the axonal support. Neuronal expression of metabolic reporters (e.g. for glucose or lactate) in CNP mutant mice and investigation of their activity upon ex-vivo stimulation of the peripheral nerves could provide more direct evidence for hampered axonal support. Moreover ex-vivo recordings of dorsal and ventral roots of CNP deficient compared to wildtype animals may give direct insight in the differential impairment of motor and sensory nerves upon CNP deficiency.

Furthermore, the exciting question of how CNP influences activity of MAPK/ERK and PI3K/AKT signaling remained unanswered. 3'5'-CAMP is reported to be promyelinating and has influence on the two pathways (Stevens et al., 2004; Monje et al., 2008). Therefore the 
catalytic activity of CNP may have an impact on the signaling activities. 2',3'-cAMP and 3',5'cAMP are positional isomers (Jackson et al., 2009) and thus the increase of 2',3'-cAMP when CNP is non-functional may also influence 3',5'-cAMP levels, which may directly activate the above mentioned signaling cascades and thus influence myelination. To this end, direct analysis of 3',5'-cAMP (and 2',3'-cAMP) level could help to unravel the link between CNP and the signaling pathways. Simultaneously this would show the importance of the CNP catalytic activity for small fiber integrity within the peripheral nervous system.

Our new observations together with potential future findings may contribute to our understanding of peripheral nerve myelination and axonal support and advance our knowledge about the etiology of peripheral neuropathies. 


\section{References}

Adlkofer K, Martini R, Aguzzi A, Zielasek J, Toyka KV, Suter U (1995) Hypermyelination and demyelinating peripheral neuropathy in Pmp22-deficient mice. Nat. Genet. 11:274-280.

Altevogt BM, Kleopa KA, Postma FR, Scherer SS, Paul DL (2002) Connexin29 Is Uniquely Distributed within Myelinating Glial Cells of the Central and Peripheral Nervous Systems. J. Neurosci. 22:6458-6470.

Armati PJ, Pollard JD (1996) Immunology of the Schwann cell. Baillières Clin. Neurol. 5:4764.

Armati PJ, Pollard JD, Gatenby P (1990) Rat and human Schwann cells in vitro can synthesize and express MHC molecules. Muscle Nerve 13:106-116.

Arthur-Farraj P, Wanek K, Hantke J, Davis CM, Jayakar A, Parkinson DB, Mirsky R, Jessen KR (2011) Mouse schwann cells need both NRG1 and cyclic AMP to myelinate. Glia 59:720-733.

Azarashvili T, Krestinina O, Galvita A, Grachev D, Baburina Y, Stricker R, Evtodienko Y, Reiser G (2009) Ca2+-dependent permeability transition regulation in rat brain mitochondria by 2',3'-cyclic nucleotides and 2',3'-cyclic nucleotide 3'-phosphodiesterase. Am. J. Physiol. - Cell Physiol. 296:C1428-C1439.

Azarashvili T, Krestinina O, Galvita A, Grachev D, Baburina Y, Stricker R, Reiser G (2014) Identification of phosphorylated form of 2', 3'-cyclic nucleotide 3'-phosphodiesterase (CNPase) as $46 \mathrm{kDa}$ phosphoprotein in brain non-synaptic mitochondria overloaded by calcium. J. Bioenerg. Biomembr. 46:135-145.

Azarashvili T, Stricker R, Reiser G (2010) The mitochondria permeability transition pore complex in the brain with interacting proteins - promising targets for protection in neurodegenerative diseases. Biol. Chem. 391:619-629.

Balice-Gordon RJ, Bone LJ, Scherer SS (1998) Functional Gap Junctions in the Schwann Cell Myelin Sheath. J. Cell Biol. 142:1095-1104.

Basson ME, Thorsness M, Finer-Moore J, Stroud RM, Rine J (1988) Structural and functional conservation between yeast and human 3-hydroxy-3-methylglutaryl coenzyme $A$ reductases, the rate-limiting enzyme of sterol biosynthesis. Mol. Cell. Biol. 8:3797-3808.

Bernier L, Alvarez F, Norgard EM, Raible DW, Mentaberry A, Schembri JG, Sabatini DD, Colman DR (1987) Molecular cloning of a 2',3'-cyclic nucleotide 3'-phosphodiesterase: mRNAs with different $5^{\prime}$ ends encode the same set of proteins in nervous and lymphoid tissues. J. Neurosci. Off. J. Soc. Neurosci. 7:2703-2710.

Bifulco M, Laezza C, Stingo S, Wolff J (2002) 2',3'-Cyclic nucleotide 3'-phosphodiesterase: A membrane-bound, microtubule-associated protein and membrane anchor for tubulin. Proc. Natl. Acad. Sci. U. S. A. 99:1807-1812. 
Birchmeier C, Nave K-A (2008) Neuregulin-1, a key axonal signal that drives Schwann cell growth and differentiation. Glia 56:1491-1497.

Blum H, Beier H, Gross HJ (1987) Improved silver staining of plant proteins, RNA and DNA in polyacrylamide gels. Electrophoresis 8:93-99.

Braun PE, De Angelis D, Shtybel WW, Bernier L (1991) Isoprenoid modification permits 2',3'cyclic nucleotide 3'-phosphodiesterase to bind to membranes. J. Neurosci. Res. 30:540544.

Braun PE, Sandillon F, Edwards A, Matthieu JM, Privat A (1988) Immunocytochemical localization by electron microscopy of 2'3'-cyclic nucleotide 3'-phosphodiesterase in developing oligodendrocytes of normal and mutant brain. J. Neurosci. Off. J. Soc. Neurosci. 8:3057-3066.

Britsch S, Goerich DE, Riethmacher D, Peirano RI, Rossner M, Nave K-A, Birchmeier C, Wegner M (2001) The transcription factor Sox10 is a key regulator of peripheral glial development. Genes Dev. 15:66-78.

Brown AM, Evans RD, Black J, Ransom BR (2012) Schwann cell glycogen selectively supports myelinated axon function. Ann. Neurol. 72:406-418.

Brushart TM (1993) Motor axons preferentially reinnervate motor pathways. J. Neurosci. 13:2730-2738.

Brushart TM, Aspalter M, Griffin JW, Redett R, Hameed H, Zhou C, Wright M, Vyas A, Höke A (2013) Schwann cell phenotype is regulated by axon modality and central-peripheral location, and persists in vitro. Exp. Neurol. 247:272-281.

Buser AM, Erne B, Werner HB, Nave K-A, Schaeren-Wiemers N (2009) The septin cytoskeleton in myelinating glia. Mol. Cell. Neurosci. 40:156-166.

Cheng CM, Reinhardt RR, Lee W-H, Joncas G, Patel SC, Bondy CA (2000) Insulin-like growth factor 1 regulates developing brain glucose metabolism. Proc. Natl. Acad. Sci. U. S. A. $97: 10236-10241$.

Cole JS, Messing A, Trojanowski JQ, Lee VM (1994) Modulation of axon diameter and neurofilaments by hypomyelinating Schwann cells in transgenic mice. J. Neurosci. 14:6956-6966.

Corfas G, Velardez MO, Ko C-P, Ratner N, Peles E (2004) Mechanisms and Roles of AxonSchwann Cell Interactions. J. Neurosci. 24:9250-9260.

Cotter L, Ozcelik M, Jacob C, Pereira JA, Locher V, Baumann R, Relvas JB, Suter U, Tricaud N (2010) Dlg1-PTEN Interaction Regulates Myelin Thickness to Prevent Damaging Peripheral Nerve Overmyelination. Science 328:1415-1418.

Court FA, Sherman DL, Pratt T, Garry EM, Ribchester RR, Cottrell DF, Fleetwood-Walker SM, Brophy PJ. 2004. Restricted growth of Schwann cells lacking Cajal bands slows conduction in myelinated nerves. Nature 431:191-195. 
Donaldson HH, Hoke GW (1905) On the areas of the axis cylinder and medullary sheath as seen in cross sections of the spinal nerves of vertebrates. J. Comp. Neurol. Psychol. 15:1-16.

Douarin NL, Dulac C, Dupin E, Cameron-Curry P (1991) Glial cell lineages in the neural crest. Glia 4:175-184.

Drummond GI, lyer NT, Keith J (1962) Hydrolysis of Ribonucleoside 2',3'-Cyclic Phosphates by a Diesterase from Brain. J. Biol. Chem. 237:3535-3539.

Drummond Gl, Perrott-Yee S (1961) Enzymatic Hydrolysis of Adenosine 3',5'-Phosphoric Acid. J. Biol. Chem. 236:1126-1129.

Edgar JM, McLaughlin M, Werner HB, McCulloch MC, Barrie JA, Brown A, Faichney AB, Snaidero N, Nave K-A, Griffiths IR (2009) Early ultrastructural defects of axons and axonglia junctions in mice lacking expression of Cnp1. Glia 57:1815-1824.

Edgar JM, McLaughlin M, Yool D, Zhang S-C, Fowler JH, Montague P, Barrie JA, McCulloch MC, Duncan ID, Garbern J, Nave KA, Griffiths IR (2004) Oligodendroglial modulation of fast axonal transport in a mouse model of hereditary spastic paraplegia. J. Cell Biol. 166:121-131.

Edgar JM, Nave K-A (2009) The role of CNS glia in preserving axon function. Curr. Opin. Neurobiol. 19:498-504.

Evangelou N, Konz D, Esiri MM, Smith S, Palace J, Matthews PM (2001) Size-selective neuronal changes in the anterior optic pathways suggest a differential susceptibility to injury in multiple sclerosis. Brain J. Neurol. 124:1813-1820.

FitzGerald MJT, Gruener G, Mtui E. 2011. Clinical Neuroanatomy and Neuroscience. Elsevier Health Sciences.

Fledrich R, Stassart RM, Klink A, Rasch LM, Prukop T, Haag L, Czesnik D, Kungl T, Abdelaal TAM, Keric N, Stadelmann C, Brück W, Nave K-A, Sereda MW (2014) Soluble neuregulin-1 modulates disease pathogenesis in rodent models of Charcot-Marie-Tooth disease 1 A. Nat. Med.

Flores Al, Narayanan SP, Morse EN, Shick HE, Yin X, Kidd G, Avila RL, Kirschner DA, Macklin WB (2008) Constitutively Active Akt Induces Enhanced Myelination in the CNS. J. Neurosci. 28:7174-7183.

Fraher JP, O'Leary D, Moran MA, Cole M, King RH, Thomas PK (1990) Relative growth and maturation of axon size and myelin thickness in the tibial nerve of the rat. 1. Normal animals. Acta Neuropathol. (Berl.) 79:364-374.

Franz CK, Rutishauser U, Rafuse VF (2005) Polysialylated neural cell adhesion molecule is necessary for selective targeting of regenerating motor neurons. J. Neurosci. Off. J. Soc. Neurosci. 25:2081-2091.

Friede RL, Bischhausen R (1980) The precise geometry of large internodes. J. Neurol. Sci. 48:367-381. 
Friede RL, Bischhausen R (1982) How are sheath dimensions affected by axon caliber and internode length? Brain Res. 235:335-350.

Fünfschilling U, Supplie LM, Mahad D, Boretius S, Saab AS, Edgar J, Brinkmann BG, Kassmann CM, Tzvetanova ID, Möbius W, Diaz F, Meijer D, Suter U, Hamprecht B, Sereda MW, Moraes CT, Frahm J, Goebbels S, Nave K-A (2012) Glycolytic oligodendrocytes maintain myelin and long-term axonal integrity. Nature 485:517-521.

Fu Q, Goodrum JF, Hayes C, Hostettler JD, Toews AD, Morell P (1998) Control of Cholesterol Biosynthesis in Schwann Cells. J. Neurochem. 71:549-555.

Garbern JY et al. (1997) Proteolipid protein is necessary in peripheral as well as central myelin. Neuron 19:205-218.

Garratt AN, Voiculescu O, Topilko P, Charnay P, Birchmeier C (2000) A Dual Role of erbB2 in Myelination and in Expansion of the Schwann Cell Precursor Pool. J. Cell Biol. 148:1035-1046.

Giese KP, Martini R, Lemke G, Soriano P, Schachner M (1992) Mouse P0 gene disruption leads to hypomyelination, abnormal expression of recognition molecules, and degeneration of myelin and axons. Cell 71:565-576.

Goebbels S, Oltrogge JH, Kemper R, Heilmann I, Bormuth I, Wolfer S, Wichert SP, Möbius W, Liu X, Lappe-Siefke C, Rossner MJ, Groszer M, Suter U, Frahm J, Boretius S, Nave KA (2010) Elevated phosphatidylinositol 3,4,5-trisphosphate in glia triggers cellautonomous membrane wrapping and myelination. J. Neurosci. Off. J. Soc. Neurosci. 30:8953-8964.

Goebbels S, Oltrogge JH, Wolfer S, Wieser GL, Nientiedt T, Pieper A, Ruhwedel T, Groszer M, Sereda MW, Nave K-A (2012) Genetic disruption of Pten in a novel mouse model of tomaculous neuropathy. EMBO Mol. Med. 4:486-499.

Gravel M, DeAngelis D, Braun PE (1994) Molecular cloning and characterization of rat brain 2',3'-cyclic nucleotide 3'-phosphodiesterase isoform 2. J. Neurosci. Res. 38:243-247

Gravel M, Gao E, Hervouet-Zeiber C, Parsons V, Braun PE (2000) Transcriptional Regulation of 2',3'-Cyclic Nucleotide 3'-Phosphodiesterase Gene Expression by Cyclic AMP in C6 Cells. J. Neurochem. 75:1940-1950.

Gravel M, Peterson J, Yong VW, Kottis V, Trapp B, Braun PE (1996) Overexpression of 2',3'Cyclic Nucleotide 3'-Phosphodiesterase in Transgenic Mice Alters Oligodendrocyte Development and Produces Aberrant Myelination. Mol. Cell. Neurosci. 7:453-466.

Gravel M, Robert F, Kottis V, Gallouzi I-E, Pelletier J, Braun PE (2009) 2',3'-Cyclic nucleotide 3'-phosphodiesterase: a novel RNA-binding protein that inhibits protein synthesis. J. Neurosci. Res. 87:1069-1079.

Griffiths I, Klugmann M, Anderson T, Yool D, Thomson C, Schwab MH, Schneider A, Zimmermann F, McCulloch M, Nadon N, Nave KA (1998) Axonal swellings and degeneration in mice lacking the major proteolipid of myelin. Science 280:1610-1613. 
Guo L, Moon C, Zheng Y, Ratner N (2013) Cdc42 regulates schwann cell radial sorting and myelin sheath folding through NF2/merlin-dependent and independent signaling. Glia 61:1906-1921.

Hagemeyer N, Goebbels S, Papiol S, Kästner A, Hofer S, Begemann M, Gerwig UC, Boretius S, Wieser GL, Ronnenberg A, Gurvich A, Heckers SH, Frahm J, Nave K-A, Ehrenreich $\mathrm{H}$ (2012) A myelin gene causative of a catatonia-depression syndrome upon aging. EMBO Mol. Med. 4:528-539.

Hahn AF, Ainsworth PJ, Bolton CF, Bilbao JM, Vallat JM (2001) Pathological findings in the $\mathrm{x}$-linked form of Charcot-Marie-Tooth disease: a morphometric and ultrastructural analysis. Acta Neuropathol. (Berl.) 101:129-139.

Hahn AF, Ainsworth PJ, Naus CC, Mao J, Bolton CF (2000) Clinical and pathological observations in men lacking the gap junction protein connexin 32. Muscle Nerve. Suppl. 9:S39-48.

Hanani M (2005) Satellite glial cells in sensory ganglia: from form to function. Brain Res. Rev. 48:457-476.

Harrisingh MC, Perez-Nadales E, Parkinson DB, Malcolm DS, Mudge AW, Lloyd AC (2004) The Ras/Raf/ERK signalling pathway drives Schwann cell dedifferentiation. EMBO J. 23:3061-3071.

Hartline DK (2008) What is myelin? Neuron Glia Biol. 4:153-163.

Hartline DK (2011) The evolutionary origins of glia. Glia 59:1215-1236.

Hartline DK, Colman DR (2007) Rapid Conduction and the Evolution of Giant Axons and Myelinated Fibers. Curr. Biol. 17:R29-R35.

Höke A, Redett R, Hameed H, Jari R, Zhou C, Li ZB, Griffin JW, Brushart TM (2006) Schwann Cells Express Motor and Sensory Phenotypes That Regulate Axon Regeneration. J. Neurosci. 26:9646-9655.

Hsieh ST, Kidd GJ, Crawford TO, Xu Z, Lin WM, Trapp BD, Cleveland DW, Griffin JW (1994) Regional modulation of neurofilament organization by myelination in normal axons. J. Neurosci. 14:6392-6401.

Hu X, Cai J, Yang J, Smith GM (2010) Sensory axon targeting is increased by NGF gene therapy within the lesioned adult femoral nerve. Exp. Neurol. 223:153-165.

Ishii A, Furusho M, Bansal $R$ (2013) Sustained Activation of ERK1/2 MAPK in Oligodendrocytes and Schwann Cells Enhances Myelin Growth and Stimulates Oligodendrocyte Progenitor Expansion. J. Neurosci. 33:175-186.

Jackson EK, Ren J, Mi Z (2009) Extracellular 2?,3?-cAMP Is a Source of Adenosine. J. Biol. Chem. 284:33097-33106.

Jahn O, Tenzer S, Werner HB (2009) Myelin Proteomics: Molecular Anatomy of an Insulating Sheath. Mol. Neurobiol. 40:55-72. 
Jessen KR (2004) Glial cells. Int. J. Biochem. Cell Biol. 36:1861-1867.

Jessen KR, Mirsky R (2005) The origin and development of glial cells in peripheral nerves. Nat. Rev. Neurosci. 6:671-682.

Jessen KR, Mirsky R (2008) Negative regulation of myelination: Relevance for development, injury, and demyelinating disease. Glia 56:1552-1565.

Jessen KR, Mirsky R (2010) Control of Schwann cell myelination. F1000 Biol. Rep. 2.

Jesuraj NJ, Nguyen PK, Wood MD, Moore AM, Borschel GH, Mackinnon SE, SakiyamaElbert SE (2012) Differential gene expression in motor and sensory Schwann cells in the rat femoral nerve. J. Neurosci. Res. 90:96-104.

Kadlubowski M, Hughes RA, Gregson NA (1984) Spontaneous and experimental neuritis and the distribution of the myelin protein P2 in the nervous system. J. Neurochem. 42:123129.

Kurihara T, Monoh K, Sakimura K, Takahashi Y (1990) Alternative splicing of mouse brain 2',3'-cyclic-nucleotide 3'-phosphodiesterase mRNA. Biochem. Biophys. Res. Commun. 170:1074-1081.

Kurihara T, Tsukada Y (1967) The regional and subcellular distribution of 2',3'-cyclic nucleotide 3'-phosphohydrolase in the central nervous system. J. Neurochem. 14:11671174.

Lappe-Siefke C, Goebbels S, Gravel M, Nicksch E, Lee J, Braun PE, Griffiths IR, Nave K-A (2003) Disruption of Cnp1 uncouples oligodendroglial functions in axonal support and myelination. Nat. Genet. 33:366-374.

Leblanc AC, Pringle J, Lemieux J, Poduslo JF, Mezei C (1992) Regulation of 2',3'-cyclic nucleotide phosphodiesterase gene expression in experimental peripheral neuropathies. Mol. Brain Res. 15:40-46.

Lee J, Gravel M, Gao E, O'Neill RC, Braun PE (2001) Identification of Essential Residues in 2',3'-Cyclic Nucleotide 3'-Phosphodiesterase. Chemical Modification and Side-Directed Mutagenesis to Investigate the Role of Cystein and Histidine Residues in Enzymatic Activity. J. Biol. Chem. 276:14804-14813.

Lee J, Gravel M, Zhang R, Thibault P, Braun PE (2005) Process outgrowth in oligodendrocytes is mediated by CNP, a novel microtubule assembly myelin protein. $\mathrm{J}$. Cell Biol. 170:661-673.

Lee J, O'Neill RC, Park MW, Gravel M, Braun PE (2006) Mitochondrial localization of CNP2 is regulated by phosphorylation of the N-terminal targeting signal by PKC: implications of a mitochondrial function for CNP2 in glial and non-glial cells. Mol. Cell. Neurosci. 31:446462.

Lee SM, Sha D, Mohammed AA, Asress S, Glass JD, Chin L-S, Li L (2013) Motor and sensory neuropathy due to myelin infolding and paranodal damage in a transgenic mouse model of Charcot-Marie-Tooth disease type 1C. Hum. Mol. Genet. 22:1755-1770. 
Lee Y, Morrison BM, Li Y, Lengacher S, Farah MH, Hoffman PN, Liu Y, Tsingalia A, Jin L, Zhang P-W, Pellerin L, Magistretti PJ, Rothstein JD (2012) Oligodendroglia metabolically support axons and contribute to neurodegeneration. Nature 487:443-448.

Lewis LN, Nunn DJ, Mezei C (1984) PO protein and 2',3'-cyclic-nucleotide 3'phosphodiesterase activity in the peripheral nerve and subcellular fractions of the Trembler mouse. J. Neurochem. 42:810-818.

Macklin WB (2010) The Myelin Brake: When Enough Is Enough. Sci. Signal. 3:pe32.

Madison RD, Sofroniew MV, Robinson GA (2009) Schwann cell influence on motor neuron regeneration accuracy. Neuroscience 163:213-221.

Madison RD, Zomorodi A, Robinson GA (2000) Netrin-1 and peripheral nerve regeneration in the adult rat. Exp. Neurol. 161:563-570.

Martini R, Schachner M, Brushart TM (1994) The L2/HNK-1 carbohydrate is preferentially expressed by previously motor axon-associated Schwann cells in reinnervated peripheral nerves. J. Neurosci. Off. J. Soc. Neurosci. 14:7180-7191.

Maurel P, Salzer JL (2000) Axonal Regulation of Schwann Cell Proliferation and Survival and the Initial Events of Myelination Requires PI 3-Kinase Activity. J. Neurosci. 20:4635-4645

Meintanis S, Thomaidou D, Jessen KR, Mirsky R, Matsas R (2001) The neuron-glia signal $\beta$ neuregulin promotes Schwann cell motility via the MAPK pathway. Glia 34:39-51.

Meyer D, Birchmeier C (1995) Multiple essential functions of neuregulin in development. Nature 378:386-390.

Michailov GV, Sereda MW, Brinkmann BG, Fischer TM, Haug B, Birchmeier C, Role L, Lai C, Schwab MH, Nave K-A (2004) Axonal Neuregulin-1 Regulates Myelin Sheath Thickness. Science 304:700-703.

Mikoshiba K, Fujishiro M, Kohsaka S, Okano H, Takamatsu K, Tsukada Y (1985) Disorders in myelination in the twitcher mutant: immunohistochemical and biochemical studies. Neurochem. Res. 10:1129-1141.

Monasterio-Schrader P, Jahn O, Tenzer S, Wichert SP, Patzig J, Werner HB (2012) Systematic approaches to central nervous system myelin. Cell. Mol. Life Sci. 69:28792894.

Monje PV, Athauda G, Wood PM (2008) Protein kinase A-mediated gating of neuregulindependent ErbB2-ErbB3 activation underlies the synergistic action of cAMP on Schwann cell proliferation. J. Biol. Chem. 283:34087-34100.

Morris JK, Lin W, Hauser C, Marchuk Y, Getman D, Lee KF (1999) Rescue of the cardiac defect in ErbB2 mutant mice reveals essential roles of ErbB2 in peripheral nervous system development. Neuron 23:273-283. 
Mortz E, Krogh TN, Vorum H, Görg A (2001) Improved silver staining protocols for high sensitivity protein identification using matrix-assisted laser desorption/ionization-time of flight analysis. Proteomics 1:1359-1363.

Muraro PA, Kalbus M, Afshar G, McFarland HF, Martin R (2002) T cell response to 2',3'cyclic nucleotide 3'-phosphodiesterase (CNPase) in multiple sclerosis patients. J. Neuroimmunol. 130:233-242.

Napoli I, Noon LA, Ribeiro S, Kerai AP, Parrinello S, Rosenberg LH, Collins MJ, Harrisingh MC, White IJ, Woodhoo A, Lloyd AC (2012) A Central Role for the ERK-Signaling Pathway in Controlling Schwann Cell Plasticity and Peripheral Nerve Regeneration In Vivo. Neuron 73:729-742.

Nave K-A (2010)(a) Myelination and the trophic support of long axons. Nat. Rev. Neurosci. $11: 275-283$.

Nave K-A (2010)(b) Myelination and support of axonal integrity by glia. Nature 468:244-252.

Nave K-A, Trapp BD (2008) Axon-Glial Signaling and the Glial Support of Axon Function. Annu. Rev. Neurosci. 31:535-561.

Newbern J, Birchmeier C (2010) Nrg1/ErbB signaling networks in Schwann cell development and myelination. Semin. Cell Dev. Biol. 21:922-928.

Newbern JM, Li X, Shoemaker SE, Zhou J, Zhong J, Wu Y, Bonder D, Hollenback S, Coppola G, Geschwind DH, Landreth GE, Snider WD (2011) Specific Functions for ERK/MAPK Signaling during PNS Development. Neuron 69:91-105.

Norton WT, Autilio LA (1965) The Chemical Composition of Bovine Cns Myelin*. Ann. N. Y. Acad. Sci. 122:77-85.

Noseda R, Belin S, Piguet F, Vaccari I, Scarlino S, Brambilla P, Boneschi FM, Feltri ML, Wrabetz L, Quattrini A, Feinstein E, Huganir RL, Bolino A (2013) DDIT4/REDD1/RTP801 Is a Novel Negative Regulator of Schwann Cell Myelination. J. Neurosci. 33:1529515305.

Oberheim NA, Wang X, Goldman S, Nedergaard M (2006) Astrocytic complexity distinguishes the human brain. Trends Neurosci. 29:547-553.

Ochoa J, Mair WG (1969) The normal sural nerve in man. I. Ultrastructure and numbers of fibres and cells. Acta Neuropathol. (Berl.) 13:197-216.

O'Neill RC, Minuk J, Cox ME, Braun PE, Gravel M (1997) CNP2 mRNA directs synthesis of both CNP1 and CNP2 polypeptides. J. Neurosci. Res. 50:248-257.

Pannese E, Rigamonti L, Procacci P, Ledda M, Arcidiacono G, Frattola D (1987) An electron microscope study of quantitative relationships between axon and Schwann cell sheath in myelinated fibres of peripheral nerves. Anat. Embryol. (Berl.) 175:423-430. 
Parkinson DB, Bhaskaran A, Arthur-Farraj P, Noon LA, Woodhoo A, Lloyd AC, Feltri ML, Wrabetz L, Behrens A, Mirsky R, Jessen KR (2008) C-Jun is a negative regulator of myelination. J. Cell Biol. 181:625-637.

Patzig J, Jahn O, Tenzer S, Wichert SP, Monasterio-Schrader P de, Rosfa S, Kuharev J, Yan K, Bormuth I, Bremer J, Aguzzi A, Orfaniotou F, Hesse D, Schwab MH, Möbius W, Nave K-A, Werner HB (2011) Quantitative and Integrative Proteome Analysis of Peripheral Nerve Myelin Identifies Novel Myelin Proteins and Candidate Neuropathy Loci. J. Neurosci. 31:16369-16386.

Peirce TR, Bray NJ, Williams NM, Norton N, Moskvina V, Preece A, Haroutunian V, Buxbaum JD, Owen MJ, O'Donovan MC (2006) Convergent evidence for 2',3'-cyclic nucleotide 3'-phosphodiesterase as a possible susceptibility gene for schizophrenia. Arch. Gen. Psychiatry 63:18-24.

Pellerin L, Pellegri G, Bittar PG, Charnay Y, Bouras C, Martin JL, Stella N, Magistretti PJ (1998) Evidence supporting the existence of an activity-dependent astrocyte-neuron lactate shuttle. Dev. Neurosci. 20:291-299.

Pereira JA, Lebrun-Julien F, Suter U (2012) Molecular mechanisms regulating myelination in the peripheral nervous system. Trends Neurosci. 35:123-134.

Pertusa M, Morenilla-Palao C, Carteron C, Viana F, Cabedo H (2007) Transcriptional control of cholesterol biosynthesis in Schwann cells by axonal neuregulin 1. J. Biol. Chem. 282:28768-28778.

Poliak S, Peles E (2003) The local differentiation of myelinated axons at nodes of Ranvier. Nat. Rev. Neurosci. 4:968-980.

Rasband MN, Tayler J, Kaga Y, Yang Y, Lappe-Siefke C, Nave K-A, Bansal R (2005) CNP is required for maintenance of axon-glia interactions at nodes of Ranvier in the CNS. Glia 50:86-90.

Readhead C, Popko B, Takahashi N, David Shine H, Saavedra RA, Sidman RL, Hood L (1987) Expression of a myelin basic protein gene in transgenic shiverer mice: Correction of the dysmyelinating phenotype. Cell 48:703-712.

Reichenbach A (1989) Glia:neuron index: review and hypothesis to account for different values in various mammals. Glia 2:71-77.

Ren J, Mi Z, Stewart NA, Jackson EK (2009) Identification and Quantification of 2',3'-cAMP Release by the Kidney. J. Pharmacol. Exp. Ther. 328:855-865.

Reynolds ES (1963) The Use of Lead Citrate at High pH as an Electron-Opaque Stain in Electron Microscopy. J. Cell Biol. 17:208-212.

Richardson KC, Jarett L, Finke EH (1960) Embedding in epoxy resins for ultrathin sectioning in electron microscopy. Stain Technol. 35:313-323. 
Riethmacher D, Sonnenberg-Riethmacher E, Brinkmann V, Yamaai T, Lewin GR, Birchmeier C (1997) Severe neuropathies in mice with targeted mutations in the ErbB3 receptor. Nature 389:725-730.

Scherer SS, Braun PE, Grinspan J, Collarini E, Wang DY, Kamholz J (1994) Differential regulation of the 2',3'-cyclic nucleotide 3'-phosphodiesterase gene during oligodendrocyte development. Neuron 12:1363-1375.

Scherer SS, Deschenes SM, Xu YT, Grinspan JB, Fischbeck KH, Paul DL (1995) Connexin32 is a myelin-related protein in the PNS and CNS. J. Neurosci. 15:8281-8294.

Schröder JM (1972) Altered ratio between axon diameter and myelin sheath thickness in regenerated nerve fibers. Brain Res. 45:49-65.

Schultz RL, Karlsson U (1965) Fixation of the Central Nervous System for Electron Microscopy by Aldehyde Perfusion. II. Effect of Osmolarity, $\mathrm{pH}$ of Perfusate and Fixative Concentration. J. Ultrastruct. Res. 12:187-206.

Shah NM, Marchionni MA, Isaacs I, Stroobant P, Anderson DJ (1994) Glial growth factor restricts mammalian neural crest stem cells to a glial fate. Cell 77:349-360.

Sheean ME, McShane E, Cheret C, Walcher J, Müller T, Wulf-Goldenberg A, Hoelper S, Garratt AN, Krüger M, Rajewsky K, Meijer D, Birchmeier W, Lewin GR, Selbach M, Birchmeier C (2014) Activation of MAPK overrides the termination of myelin growth and replaces Nrg1/ErbB3 signals during Schwann cell development and myelination. Genes Dev. 28:290-303.

Sherman DL, Brophy PJ. 2005. Mechanisms of axon ensheathment and myelin growth. Nat Rev Neurosci 6:683-690.

Sherwood CC, Stimpson CD, Raghanti MA, Wildman DE, Uddin M, Grossman LI, Goodman M, Redmond JC, Bonar CJ, Erwin JM, Hof PR (2006) Evolution of increased glia-neuron ratios in the human frontal cortex. Proc. Natl. Acad. Sci. 103:13606-13611.

Smith RS, Koles ZJ (1970) Myelinated nerve fibers: computed effect of myelin thickness on conduction velocity. Am. J. Physiol. 219:1256-1258.

Snaidero N, Möbius W, Czopka T, Hekking LHP, Mathisen C, Verkleij D, Goebbels S, Edgar J, Merkler D, Lyons DA, Nave K-A, Simons M (2014) Myelin membrane wrapping of CNS axons by $\mathrm{PI}(3,4,5) \mathrm{P3}-d e p e n d e n t$ polarized growth at the inner tongue. Cell 156:277-290.

Sprinkle TJ, Zaruba ME, McKhann GM (1978)(a) Activity of 2',3'-cyclic-nucleotide 3'phosphodiesterase in regions of rat brain during development: quantitative relationship to myelin basic protein. J. Neurochem. 30:309-314.

Sprinkle TJ, Zaruba ME, McKhann GM (1978)(b) Radioactive measurement of 2' ,3'-cyclic nucleotide 3'-phosphodiesterase activity in the central and peripheral nervous system and in extraneural tissue. Anal. Biochem. 88:449-456. 
Stevens B, Ishibashi T, Chen J-F, Fields RD (2004) Adenosine: an activity-dependent axonal signal regulating MAP kinase and proliferation in developing Schwann cells. Neuron Glia Biol. 1:23-34.

Stone TW, Ceruti S, Abbracchio MP (2009) Adenosine Receptors and Neurological Disease: Neuroprotection and Neurodegeneration In C. N. Wilson \& S. J. Mustafa, eds. Adenosine Receptors in Health and Disease Handbook of Experimental Pharmacology. Springer Berlin Heidelberg, p. 535-587.

Suda H, Hosokawa T, Ohno R, Hamaguchi K, Tsukada Y (1984) 2',3'-Cyclic nucleotide 3'phosphodiesterase activity in the cerebrospinal fluid of patients with demyelinating diseases. Neurochem. Pathol. 2:85-102.

Taveggia C, Zanazzi G, Petrylak A, Yano H, Rosenbluth J, Einheber S, Xu X, Esper RM, Loeb JA, Shrager P, Chao MV, Falls DL, Role L, Salzer JL (2005) Neuregulin-1 Type III Determines the Ensheathment Fate of Axons. Neuron 47:681-694.

Thompson JE, Venegas FD, Raines RT (1994) Energetics of catalysis by ribonucleases: fate of the 2',3'-cyclic phosphodiester intermediate. Biochemistry (Mosc.) 33:7408-7414.

Trapp BD, Bernier L, Andrews SB, Colman DR (1988) Cellular and subcellular distribution of 2',3'-cyclic nucleotide 3'-phosphodiesterase and its mRNA in the rat central nervous system. J. Neurochem. 51:859-868.

Tricaud N, Perrin-Tricaud C, Brusés JL, Rutishauser U (2005) Adherens Junctions in Myelinating Schwann Cells Stabilize Schmidt-Lanterman Incisures via Recruitment of p120 Catenin to E-Cadherin. J. Neurosci. 25:3259-3269.

Vega C, Martiel J-L, Drouhault D, Burckhart M-F, Coles JA (2003) Uptake of locally applied deoxyglucose, glucose and lactate by axons and Schwann cells of rat vagus nerve. J. Physiol. 546:551-564.

Verheijen MHG, Camargo N, Verdier V, Nadra K, de Preux Charles A-S, Médard J-J, Luoma $A$, Crowther $M$, Inouye $H$, Shimano $H$, Chen $S$, Brouwers JF, Helms JB, Feltri ML, Wrabetz L, Kirschner D, Chrast R, Smit AB (2009) SCAP is required for timely and proper myelin membrane synthesis. Proc. Natl. Acad. Sci. U. S. A. 106:21383-21388.

Verkhratsky A, Butt AM (2013) Glial Physiology and Pathophysiology. Chapter 3 Neuroglia; Wiley-Blackwell.

Verrier JD, Jackson TC, Bansal R, Kochanek PM, Puccio AM, Okonkwo DO, Jackson EK (2012) The brain in vivo expresses the 2',3'-cAMP-adenosine pathway. J. Neurochem.

Verrier JD, Jackson TC, Gillespie DG, Janesko-Feldman K, Bansal R, Goebbels S, Nave KA, Kochanek PM, Jackson EK (2013) Role of CNPase in the oligodendrocytic extracellular 2',3'-cAMP-adenosine pathway. Glia 61:1595-1606.

Viader A, Golden JP, Baloh RH, Schmidt RE, Hunter DA, Milbrandt J (2011) Schwann Cell Mitochondrial Metabolism Supports Long-Term Axonal Survival and Peripheral Nerve Function. J. Neurosci. 31:10128 -10140. 
Vlkolinský R, Cairns N, Fountoulakis M, Lubec G (July) Decreased brain levels of 2',3'-cyclic nucleotide-3'-phosphodiesterase in Down syndrome and Alzheimer's disease. Neurobiol. Aging 22:547-553.

Waxman SG (1980) Determinants of conduction velocity in myelinated nerve fibers. Muscle Nerve 3:141-150.

Werner HB, Kuhlmann K, Shen S, Uecker M, Schardt A, Dimova K, Orfaniotou F, Dhaunchak A, Brinkmann BG, Möbius W, Guarente L, Casaccia-Bonnefil P, Jahn O, Nave K-A. 2007. Proteolipid Protein Is Required for Transport of Sirtuin 2 into CNS Myelin. J Neurosci 27:7717-7730.

Whitfeld PR, Heppel LA, Markham R (1955) The enzymic hydrolysis of ribonucleoside-2':3' phosphates. Biochem. J. 60:15-19.

Wieser GL, Gerwig UC, Adamcio B, Barrette B, Nave K-A, Ehrenreich H, Goebbels S (2013) Neuroinflammation in white matter tracts of Cnp1 mutant mice amplified by a minor brain injury. Glia 61:869-880.

Williams PL, Wendell-Smith CP (1971) Some additional parametric variations between peripheral nerve fibre populations. J. Anat. 109:505-526.

Woodhoo A, Alonso MBD, Droggiti A, Turmaine M, D’Antonio M, Parkinson DB, Wilton DK, Al-Shawi R, Simons P, Shen J, Guillemot F, Radtke F, Meijer D, Feltri ML, Wrabetz L, Mirsky R, Jessen KR (2009) Notch controls embryonic Schwann cell differentiation, postnatal myelination and adult plasticity. Nat. Neurosci. 12:839-847.

Xu K, Terakawa S (1999) Fenestration nodes and the wide submyelinic space form the basis for the unusually fast impulse conduction of shrimp myelinated axons. J. Exp. Biol. 202:1979-1989.

Ydens E, Lornet G, Smits V, Goethals S, Timmerman V, Janssens S (2013) The neuroinflammatory role of Schwann cells in disease. Neurobiol. Dis. 55:95-103.

Yin X, Crawford TO, Griffin JW, Tu P, Lee VM-Y, Li C, Roder J, Trapp BD (1998) MyelinAssociated Glycoprotein Is a Myelin Signal that Modulates the Caliber of Myelinated Axons. J. Neurosci. 18:1953-1962.

Yin X, Peterson J, Gravel M, Braun PE, Trapp BD (1997) CNP overexpression induces aberrant oligodendrocyte membranes and inhibits MBP accumulation and myelin compaction. J. Neurosci. Res. 50:238-247. 